\title{
Code Design for Incremental Redundancy Hybrid ARQ
}

\author{
by
}

Hamid Saber

\author{
A thesis submitted to the \\ Faculty of Graduate and Postdoctoral Affairs \\ in partial fulfillment of the requirements for the degree of
}

Doctor of Philosophy in Electrical and Computer Engineering

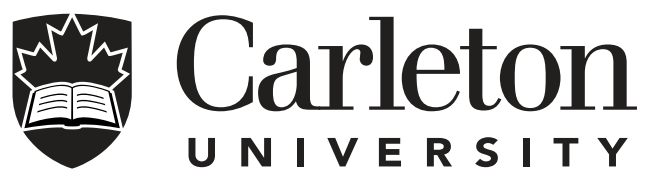

Ottawa-Carleton Institute for Electrical and Computer Engineering

Department of Systems and Computer Engineering

\author{
Carleton University \\ Ottawa, Ontario
}

August, 2016

(C)Copyright

Hamid Saber, 2016 


\section{Abstract}

In this work, we study the problem of designing rate-compatible (RC) error correcting codes for use in incremental redundancy hybrid ARQ (IR-HARQ) systems to address the rate flexibility requirement of wireless communication systems. Our goal is to design codes to maximize the throughput of IR-HARQ, where the throughput is defined as the number of the bits in a message divided by the average number of code bits that need to be transmitted for successful decoding. The rate-flexibility of our schemes is achieved by puncturing and extending a mother code. We consider reliability-based (RB) HARQ schemes where a feedback channel is used to convey information reflecting the reliability of the received code bits. We aim to design RB-HARQ schemes based on LDPC codes with the goal of improving the throughput performance while maintaining the overhead in the feedback channel. We then show how both low density parity check (LDPC) and low density generator matrix (LDGM) codes can be combined to design RC codes whose nature varies from LDPC to LDGM as the rate of the codes decreases, and thus benefiting from the advantages of both types of codes at the same time. The proposed method results in a universal capacity-approaching IR-HARQ scheme which remains within $1 \mathrm{~dB}$ of the Shannon capacity of the binary input additive white Gaussian noise (BIAWGN) channel. We then study the design of polar codes for IR-HARQ. We propose new puncturing and extending algorithms for polar codes, and show how they can result in capacity-approaching throughput performance with very low decoding complexity. We then aim to improve the performance of polar codes at finite lengths to use them as the mother code. In particular, the design of generalized concatenated codes based on polar (GCC-polar) codes is studied. A new method to design the GCC-polar codes is proposed. The proposed method employs density evolution to design the outer codes for the actual channels seen by them with the goal of minimizing their BLER. Once a set of outer codes with different rates have been constructed, we propose a rate-allocation algorithm to determine the rates of the outer codes of the GCC-polar code. The resulting GCC-polar codes outperform Arikan's codes and the previous works on the literature and can be used in place of the mother code for IR-HARQ 
based on polar codes. 
To My Parents 


\section{Acknowledgments}

I would like to express my deepest gratitude to my supervisor, Dr. Ian Marsland, for his invaluable guidance and close engagement throughout the course of my research. He has been a great mentor to me without whom none of this would have been possible. It has been an honor for me to have had the pleasure of working with him.

I am mostly grateful to my parents for their invaluable support and faith in me. I would also like to thank all my friends in Ottawa and at Carleton University who made this journey possible. 


\section{Table of Contents}

Abstract

Acknowledgments $\quad$ v

Table of Contents vi

List of Tables $\quad$ ix

List of Figures $\quad$ x

Acronyms $\quad$ xii

1 Introduction 1

1.1 Publications . . . . . . . . . . . . . . . . . . 4

1.2 Organization of the thesis ................ 4

2 Existing Code Construction Methods for IR-HARQ 6

2.1 Good RC codes for IR-HARQ . . . . . . . . . . . . . . . 6

2.2 Reliability-based IR-HARQ . . . . . . . . . . . . . . . . 7

2.3 LDPC Codes for IR-HARQ . . . . . . . . . . . . . . . . . . . . . . 9

2.4 LDGM codes for IR-HARQ . . . . . . . . . . . . . . . . 12

2.5 Polar Codes for IR-HARQ . . . . . . . . . . . . . . . . . . . . 14

2.6 Summary .............................. 16

3 Reliability-based IR-HARQ 18

3.1 Code Structure . . . . . . . . . . . . . . . . . . . . 18

3.2 Reliability Metrics . . . . . . . . . . . . . . . . 20

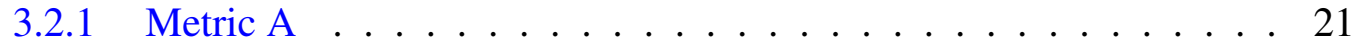

3.2 .2 Metric B ..................... 22 
3.2 .3 Metric $\mathrm{C} \ldots \ldots \ldots \ldots \ldots \ldots \ldots$

3.3 Simulation Results . . . . . . . . . . . . . . . . . . . . 24

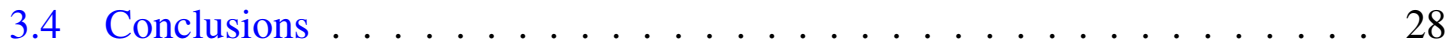

4 Combined LDPC and LDGM Codes for IR-HARQ 29

4.1 Introduction . . . . . . . . . . . . . . . . . . 29

4.2 LDGM Codes . . . . . . . . . . . . . . . . . . . 30

4.3 Proposed RC LDPC/LDGM Code Construction . . . . . . . . . . . . . . 32

4.3.1 Construction of the generator matrix $\mathbf{G}_{N_{h}} \ldots \ldots . \ldots 33$

4.3.2 Construction of the extended generator matrices, $\mathbf{G}_{n} \ldots \ldots 35$

4.4 Decoding of RC LDPC/LDGM Codes . . . . . . . . . . . . . . 36

4.4.1 Parity-Check Decoding of the LDGM Codes $C_{n}, n>N_{h} \ldots 36$

4.4.2 Combined Decoding . . . . . . . . . . . . . . 40

4.4 .3 IR-HARQ scheme ...................... 41

4.5 Simulation Results . . . . . . . . . . . . . . . . . . . . 42

4.6 Conclusion . . . . . . . . . . . . . . . . . 48

5 Polar Codes for IR-HARQ 49

5.1 Introduction . . . . . . . . . . . . . . . . . . 49

5.2 Polar codes . . . . . . . . . . . . . . . 50

5.2 .1 Encoding and Decoding ............... 51

5.2 .2 Code Design . . . . . . . . . . . . . . . 54

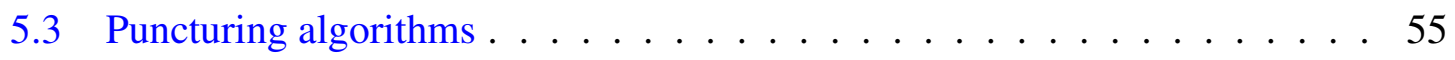

5.4 Extending algorithms . . . . . . . . . . . . . 57

5.4.1 Proposed Algorithm . . . . . . . . . . . . . . 58

5.4.2 A Less Greedy Extending Algorithm . . . . . . . . . . . . . 63

5.4 .3 The proposed IR-HARQ scheme . . . . . . . . . . . 64

5.5 Simulation Results . . . . . . . . . . . . . . . . 65

5.6 Conclusion .......................... 71

6 Design of Generalized Concatenated Polar Codes 73

6.1 Outer codes of a Polar Code . . . . . . . . . . . . . . 75

6.2 Proposed Method for Designing GCC-Polar Codes . . . . . . . . . . . . 76

6.2.1 Design of outer codes . . . . . . . . . . . . . 78

6.2.2 Rate allocation algorithm . . . . . . . . . . . 83 
6.2.3 Decoding Complexity . . . . . . . . . . . . 85

6.3 Performance Evaluation Results . . . . . . . . . . . . . . . 88

6.4 Conclusion . . . . . . . . . . . . . . . . . 95

7 Conclusion and Future Works 96

8 Appendix 99

8.0.1 Linear Block Codes over BIMOS Channels: An ML Decoder and an Upper Bound on their BLER . . . . . . . . . . . . . . 99

8.0.2 Minimal Trellises of the Designed Outer Codes of Length $L=8$. . 101 


\section{List of Tables}

6.1 The number of operations required for decoding Arikan's outer codes under SC decoding and the designed outer codes under ML decoding: The four types of operations are addition + , comparison $\leq$, product $\times$ and tanh evaluation. . . . . . . . . . . . . . . . 86

6.2 Outer code rate distributions $\Omega_{\omega}$ for conventional and GCC-polar codes. . . 87

6.3 The average number of performed operations for outer code decodings of the codes in Fig. 6.5 . . . . . . . . . . . . . . . . . . 87

6.4 Outer code rate distributions $\Omega_{\omega}$ for GCC-polar codes using the rate allocation algorithm in [86]. . . . . . . . . . . . . . . . . . 92

6.5 The average number of performed operations for outer code decodings of the codes in Table 6.4. . . . . . . . . . . . . . . . . . . . 92 


\section{List of Figures}

2.1 Throughput of a reliability-based IR-HARQ based on LDPC codes according to $[13] . \ldots \ldots \ldots \ldots \ldots$

2.2 The throughput of IR-HARQ with LDPC codes using the puncturing method in [36] for different mother code rates. . . . . . . . . . . . . 10

2.3 The throughput of IR-HARQ based on [24] and Ha's puncturing method [33]. 11

2.4 Throughput of IR-HARQ with Raptor codes. . . . . . . . . . . . . . . . 14

2.5 Throughput of IR-HARQ based on Polar codes constructed according to [71]. 16

3.1 Parity check matrix structure of the extended codes. . . . . . . . . . . . 20

3.2 Throughput of the proposed RB-HARQ system with the three different reliability metrics. . . . . . . . . . . . . . . . . . 25

3.3 The effect of the cluster size on the throughput of the proposed RB-HARQ system. . . . . . . . . . . . . . . . 26

3.4 Throughput after compensating for the feedback overhead. . . . . . . . . . 27

4.1 Generator graph of a sample LDGM code. . . . . . . . . . . . . . . . 31

4.2 BLER of the two decoding methods for the code sequence $\left\{C_{n}\right\}$ in terms of their corresponding rates. . . . . . . . . . . . . . . . . . 39

4.3 BLER of the three decoding methods for the code sequence $\left\{C_{n}\right\}$ in terms of their corresponding rates. . . . . . . . . . . . . . . . . . . 41

4.4 The effect of the rate, $R_{h}$, of the LDPC mother code on the throughput performance of an IR-HARQ $\left(R_{h}, R_{i}, R_{g}\right)$ system. . . . . . . . . . . 43

4.5 The effect of the rate, $R_{g}$, of the LDGM code on the the throughput performance of an IR-HARQ $\left(R_{h}, R_{i}, R_{g}\right)$ system. . . . . . . . . . . . . . 44

4.6 The effect of the rate $R_{i}$ on the the throughput performance of an IR$\operatorname{HARQ}\left(R_{h}, R_{i}, R_{g}\right)$ system. . . . . . . . . . . . . . . 45

4.7 Throughput result of different IR-HARQ schemes. . . . . . . . . . . . . 46

4.8 Throughput result of different IR-HARQ schemes. . . . . . . . . . . . . . 47

5.1 The PC graph of a polar code with length $N=8 \ldots \ldots \ldots 2$ 
5.2 The BLERs of punctured polar codes with different puncturing algorithms. . 66

5.3 Throughput of the proposed IR-HARQ scheme with the proposed puncturing algorithm for different $R_{M}$, all with $R_{I}=R_{M} \ldots$. . . . . . . . . . . 67

5.4 Throughput of the proposed IR-HARQ scheme with the proposed puncturing and extending algorithm with different $R_{I}$ s, with $R_{M}=0.5$. . . . . . 68

5.5 Throughput comparison of the proposed IR-HARQ scheme with other alternatives. . . . . . . . . . . . . . . . . .

5.6 Throughput of the proposed system for different number of decodings with a cluster size of $S=32 \ldots \ldots \ldots \ldots$. . . . . . . . . . 71

5.7 Throughput of the proposed IR-HARQ scheme for different lengths of the mother polar code, with $R_{M}=0.5$ and $R_{I}=1$. . . . . . . . . . . . . . 72

6.1 The PC graph of the polar code of length $N=8$ and its outer codes of lengths $L=1,2$, and $4 \ldots \ldots \ldots \ldots$

6.2 The encoding and decoding graph of a GCC-polar code of length $N=2^{n}$ with a set of $\left(L=2^{l}, \omega_{k}\right)$ outer codes $C_{L, k} \ldots \ldots \ldots$. . . . . . . 77

6.3 The performance of the designed outer codes (solid lines) versus Arikan's (dashed lines) over the BI-AWGN channel, for different code rates. . . . . . 82

6.4 The performance comparison of the GCC-polar code (solid lines) and the conventional polar code (dashed lines) for different code rates, with $N=$ 256 and $L=8$. . . . . . . . . . . . . . . . . . . . . . . . . . . 88

6.5 The performance comparison of the GCC-polar code (solid lines) and the conventional polar code (dashed lines) for different code rates, with $N=$ 1024 and $L=8$.

6.6 The impact of the rate allocation algorithm on the performance of GCCpolar codes. Solid lines correspond to the proposed rate allocation algorithm while the dashed lines correspond to the equal error probability rule.

6.7 Block error rates of Arikan and GCC-polar codes under SC and CA-SCL decoding with a list size of $32 \ldots \ldots . \ldots . \ldots$. . . . . . . . . . 93

8.1 The minimal trellis of $\mathbf{G}_{1} \ldots \ldots \ldots$. . . . . . . . . . . . . . . . . . .

8.2 The minimal trellis of $\mathbf{G}_{2} \ldots \ldots \ldots \ldots$. . . . . . . . . . . . . . . . .

8.3 The minimal trellis of $\mathbf{G}_{3} \ldots \ldots \ldots$. . . . . . . . . . . . . . . . . . . .

8.4 The minimal trellis of $\mathbf{G}_{4} \ldots \ldots \ldots \ldots$. . . . . . . . . . . . . . . . . . . . .

8.5 The minimal trellis of $\mathbf{G}_{5} \ldots \ldots \ldots \ldots$. . . . . . . . . . . . . . . . . . .

8.6 The minimal trellis of $\mathbf{G}_{6} \ldots \ldots \ldots \ldots$ 


\section{Acronyms}

$\begin{array}{ll}\text { ARQ } & \text { Automatic Repeat Request } \\ \text { AWGN } & \text { Additive White Gaussian Noise } \\ \text { BEC } & \text { Binary Erasure Channel } \\ \text { BER } & \text { Bit Error Rate } \\ \text { BIAWGN } & \text { Binary Input Additive White Gaussian Noise } \\ \text { BIMOS } & \text { Binary Input Memoryless Output Symmetric } \\ \text { BLER } & \text { Block Error Rate } \\ \text { BP } & \text { Belief Propagation } \\ \text { BPSK } & \text { Binary Phase Shift Keying } \\ \text { CA-SCL } & \text { CRC-Aided Successive Cancellation List } \\ \text { CRC } & \text { Cyclic Redundancy Check } \\ \text { DE } & \text { Density Evolution } \\ \text { GA } & \text { Gaussian Approximation } \\ \text { GCC } & \text { Generalized Concatenated Code } \\ \text { HARQ } & \text { Hybrid Automatic Repeat Request } \\ \text { IR } & \text { Incremental Redundancy } \\ \text { IRA } & \text { Irregular Repeat Accumulate } \\ \text { LDGM } & \text { Low Density Generator Matrix } \\ \text { LDPC } & \text { Low Density Parity Check } \\ & \end{array}$




$\begin{array}{ll}\text { LLR } & \text { Log-likelihood Ratio } \\ \text { MAP } & \text { Maximum a Posteriori } \\ \text { ML } & \text { Maximum Likelihood } \\ \text { MRC } & \text { Maximum Ratio Combining } \\ \text { MSGM } & \text { Minimum Span Generator Matrix } \\ \text { PC } & \text { Polar Code } \\ \text { PEG } & \text { Progressive Edge Growth } \\ \text { QUP } & \text { Quasi-Uniform Puncturing } \\ \text { RB } & \text { Reliability Based } \\ \text { RB-HARQ } & \text { Reliability-Based Hybrid Automatic Repeat Request } \\ \text { RC } & \text { Rate-Compatible } \\ \text { SC } & \text { Successive Cancellation } \\ \text { SNR } & \text { Signal-to-Noise Ratio }\end{array}$




\section{Chapter 1}

\section{Introduction}

The received signal-to-noise ratio (SNR) in wireless communications systems typically fluctuates over time because of changes in the channel path loss, shadowing, and fading. It is therefore necessary to deploy transmission schemes that can operate reliably over a wide range of SNRs.

To ensure reliable transmission, an automatic repeat request (ARQ) scheme can be used. ARQ is an error control strategy in which the transmitter uses an error detecting code, such as a cyclic redundancy check (CRC) code, to encode the message bits prior to transmission. The receiver uses the code to detect whether or not the message was received correctly, and if it was not, the receiver asks the transmitter to retransmit the message.

One problem with ARQ is that if the channel is poor (i.e., the SNR is low) then it is possible that the message will never be received correctly, regardless of how many times it is retransmitted. It is possible to improve ARQ by employing both an error correcting code and an error detecting code in a hybrid ARQ (HARQ) scheme. When an error correcting code is used, the transmitter adds carefully controlled redundancy to the message and transmits the resulting codeword. The receiver uses the error correcting code to correct as many transmission errors as possible. The error detecting code detects whether or not any error remains, and if the message is still not received error-free, the receiver requests retransmission of the encoded message, just like with standard ARQ. HARQ is able to increase the throughput, defined as the number of bits in a message divided by the average number of code bits that need to be transmitted for successful decoding, at low SNRs because the number of needed retransmissions is reduced. However, the throughput at high SNRs is decreased because the additional redundancy associated with the error correcting code is not needed but transmitted anyway.

If the SNR is known at the transmitter prior to transmission, an adaptive modulation and 
coding scheme can be used to reduce the inefficiency of HARQ. When the channel is good only a little bit (if any) of redundancy is added, and when the channel is poor a lot is added (increasing the amount of redundancy increases the likelihood that the message will be decoded successfully, but also decreases the throughput). This widely-used technique improves the efficiency of HARQ, but it does require the knowledge of the SNR at the transmitter. Since perfect knowledge of what the SNR will be once transmission commences is impossible to attain, only an estimate is available. It is therefore advisable to incorporate a small margin of error and transmit a little more redundancy that is strictly needed, thereby slightly reducing the efficiency. More importantly, though, the need to retransmit the whole codeword on decoding failure means that it is important to carefully balance the amount of redundancy added with the cost of retransmission.

To avoid the need for SNR knowledge at the transmitter, and to reduce the retransmission cost, an enhanced type of HARQ, known as incremental redundancy HARQ (IR-HARQ) can be used. With IR-HARQ, the transmitter encodes the message with a high-rate error correcting code (i.e., only a little redundancy is added) and sends the encoded message to the receiver. This is to ensure that no additional resources are wasted in the case that the channel is very good. The decoder attempts to decode the message word by decoding the code. If decoding fails, the transmitter produces additional redundancy and sends it to the receiver. The receiver attempts to recover the message bits by combining the previously received codeword with these incremental redundancy bits. If the decoding still fails, even more redundancy bits are sent to the receiver. This process continues until the message is decoded successfully.

It is possible to improve the throughput of IR-HARQ by informing the transmitter via a feedback channel which redundancy code bits are most beneficial for retransmission. One approach is to inform the transmitter about the reliability of the received code bits in a reliability-based HARQ (RB-HARQ) scheme. With RB-HARQ the receiver measures the reliability of the received code bits using a reliability metric and then asks the transmitter for the retransmission of those code bits with the least reliability. RB-HARQ is able to improve the throughput of IR-HARQ. However, this comes at the expense of a feedback overhead due to the transmission on the feedback channel, which needs to be maintained for practical systems.

The main drawback of ARQ schemes is the latency introduced due to the transmission of acknowledgement from the receiver. Although it is possible to reduce this latency by 
increasing the granularity of the system through increasing the amount of transmitted redundancy when required by the receiver, this comes at the expense of a degradation in throughput which in turn reduces the efficiency. Whether or not an HARQ scheme can be used in practice significantly depends on the latency requirements of the communication system. Nowadays HARQ is used in practical communication systems such as HSDPA and HSUPA for mobile phone networks and in the IEEE 802.16-2005 WIMAX mobile wireless access.

Rate-compatible (RC) codes are usually used with IR-HARQ to provide incremental redundancy to the receiver. RC codes are a sequence of channel codes for which the code bits of a higher-rate code are embedded in those of the lower-rate code. That is, once a codeword of a specific code rate has been transmitted over the channel, sending additional code bits can realize the transmission of the codeword of the next lower-rate code. The importance of the RC codes lies in their ability to be implemented with a single encoder and decoder, which in turn reduces the encoding and decoding complexity to a significant extent.

As mentioned before, the performance of IR-HARQ is measured in terms of throughput. In the context of IR-HARQ, it is desired to design RC codes with efficient encoding and decoding complexity such that the throughput is maximized. For a given length of the message word, this is equivalent to minimizing the average number of code bits that must be transmitted for successful decoding. This quantity is a function of the sequence of RC codes. In the proposed research our goal is to design good RC codes for use with IR-HARQ in such a way that the throughput is maximized.

Construction of RC codes usually starts from a mother code. Puncturing is the most common way to produce RC codes. With puncturing, some of the code bits of the mother code are not transmitted over the channel (i.e., they are punctured). This will result in a code whose rate is higher than that of the mother code. The lowest achievable rate with puncturing without resorting to retransmission of code bits is that of the mother code. Therefore to get very low rates, a low rate mother code has to be used. On the other hand it is known that puncturing of a low-rate mother code is not capable of producing good highrate codes. Instead it is better to use a medium-rate mother code that can be punctured to higher rates and extended to lower rates. Extending a linear block code simply means to add columns to its generator matrix. These columns can produce additional codes bits required for getting lower-rate codes. We consider different classes of mother codes and different 
methods of constructing RC codes, including puncturing and extending, for IR-HARQ systems. Specifically mother codes from the following classes are considered: Low-density parity-check (LDPC), low-density generator-matrix (LDGM), LT, Raptor, and the recentlyproposed capacity-achieving polar codes. Different methods to construct RC codes from each class are studied, and the throughput of IR-HARQ using these codes is investigated.

\subsection{Publications}

The work of this thesis has resulted in the following publications.

[C1] Hamid Saber and Ian Marsland, "A novel hybrid ARQ scheme based on LDPC code extension and feedback," in Proc. Vehicular Technology Conference (VTC Fall), Quebec City, Canada, Sept. 2012.

[C2] Hamid Saber and Ian Marsland, "A new reliability-based hybrid ARQ scheme based on LDPC codes," in Proc. Canadian Workshop on Information Theory (CWIT) 2015, St. Johns, Canada, July 2015.

[J1] Hamid Saber and Ian Marsland, "An incremental redundancy hybrid AQR scheme based on puncturing and extending of polar codes," IEEE Transactions on Communications, vol. 63, no. 11, pp. 3964-3973, Nov. 2015.

[J2] Hamid Saber and Ian Marsland, "Design of generalized concatenated codes based on polar codes with very short outer codes," accepted for publication in IEEE Transactions on Vehicular Technology, July 2016.

[J3] Hamid Saber and Ian Marsland, "An incremental redundancy hybrid ARQ scheme based on combined LDPC and LDGM codes", Under review in IEEE Transactions on Vehicular Technology

\subsection{Organization of the thesis}

The rest of this thesis is organized as follows. In the next chapter we give a review of the different existing methods for constructing $\mathrm{RC}$ codes, for the different classes of mother 
codes mentioned above. We then show their throughput performance analysis and highlight their advantages and disadvantages. Chapter 3 presents a new RB-HARQ scheme based on LDPC codes. Different metrics to evaluate the reliability of clusters of codes bits are proposed and employed in the RB-HARQ scheme. By combining LDPC and LDGM codes in Chapter 4 we design RC codes whose nature changes from LDPC to LDGM as the code rate decreases. We show how it can result in a universal capacity approaching IR-HARQ scheme while maintaining low encoding and decoding complexity. Chapter 5 studies the design of polar codes for IR-HARQ. We propose algorithms for both puncturing and extending of polar codes, and show how these algorithms can be used to yield a capacity approaching IR-HARQ scheme. In Chapter 6 we aim to improve the performance of polar mother codes at finite lengths. In particular, the design of generalized concatenated codes based on polar codes is studied. The thesis is concluded in Chapter 7. 


\section{Chapter 2}

\section{Existing Code Construction Methods for IR-HARQ}

\subsection{Good RC codes for IR-HARQ}

Suppose that a message word consisting of $K$ bits is to be transmitted to the receiver. Let $\left\{C_{i} \mid i=1,2, \ldots\right\}$ be a sequence of codes, where $C_{i}$ has code length $N_{i}$ for which $K \leq N_{1} \leq$ $N_{2} \leq \ldots$. The $i$-th code rate is $R_{i}=\frac{K}{N_{i}}$. Let $\mathbf{G}^{(i)}$ be the $K \times N_{i}$ generator matrix of $C_{i}$. We say that a sequences of codes with dimension $K$ and code lengths $\left\{N_{i}\right\}$ are rate compatible if the following holds for the generator matrices for $i \geq 2$ :

$$
\mathbf{G}^{(i)}=\left[\begin{array}{ll}
\mathbf{G}^{(i-1)} & \mathbf{G}_{E}^{(i)}
\end{array}\right]
$$

for some extending matrix $\mathbf{G}_{E}^{(i)}$. With an IR-HARQ scheme the message word $\mathbf{u}=$ $\left[u_{1}, \ldots, u_{K}\right]$ is encoded by the first RC code, of length $N_{1}$, and the codeword $\mathbf{u G}^{(1)}$ is transmitted over the channel. The decoder for $C_{1}$ attempts to decode the received word. If it fails, new code bits $\mathbf{u G}_{E}^{(2)}$ are generated via the extending matrix $\mathbf{G}_{E}^{(2)}$ and sent over the channel. This realizes the transmission of the codeword of $C_{2}$. If decoding still fails, $\mathbf{u G}_{E}^{(3)}$ is transmitted, and decoding is attempted for $C_{3}$. This process continues until decoding is successful. Let $\bar{N}$ be the average number of code bits required for successful decoding. The throughput of the IR-HARQ system is then defined as

$$
\eta=\frac{K}{\bar{N}}=\frac{K}{\sum_{i} N_{i} P_{i}}
$$


where $P_{i}$ is the probability that all the decodings of the codes $\left\{C_{j} \mid j<i\right\}$ fail, but that of code $C_{i}$ succeeds.

Obviously, for channels described by a parameter, the throughput is a function of the channel parameter. For example, this parameter can be the SNR in the case of the binary input additive white Gaussian noise (BI-AWGN) channel. For any specific channel, it is desired that the throughput be as close as possible to the channel capacity. For the channels that are described by a channel parameter, it is desired that the throughput remains as close as possible to the capacity for a wide range of the channel parameter. For example, consider a BI-AWGN channel with binary phase shift keying (BPSK). It is desired to design the RC codes in such a way that the throughput of the corresponding IR-HARQ scheme is as close to the capacity as possible for all SNRs. Among the first works on designing IR-HARQ schemes based on well-known channel codes are [1]- [12], in which codes from the class of convolutional codes were used. The schemes which use convolutional and turbo codes are known to be inferior to those based on LDPC codes and thus are not presented here. In the following we review the design of LDPC, LDGM, and polar codes for IR-HARQ.

\subsection{Reliability-based IR-HARQ}

Reliability-based hybrid ARQ (RB-HARQ) was introduced by Shea in [13]. RB-HARQ is a type of selective ARQ in which the receiver, based on information extracted from the received codeword reflecting the reliability of the received code bits, requests retransmission of only those code bits which were weakly received. RB-HARQ has been used with convolutional codes [14]- [16] and LDPC codes ( [17]) [18]- [19]. Substantial performance improvements over the traditional hybrid ARQ schemes were reported. Fig. 2.1 shows the throughput of an RB-HARQ scheme based on LDPC codes with a reliability metric calculated according to [13]. In the event of a decoding failure the receiver requests retransmission of one code bit. This code bit is chosen according to a reliability metric at the receiver. The metric used in [13] is the absolute value of the LLR of the received code bits. That is, the code bit with the smallest absolute value of LLR is requested for a retransmission. Maximum ratio combining (MRC) [20] is used to combine the LLRs of repeatedly received code bits. As can be seen the resulting RB-HARQ system is superior to the traditional non-reliability based (Non-RB) HARQ scheme based on LDPC codes, with over 2 $\mathrm{dB}$ gain at low SNRs. 


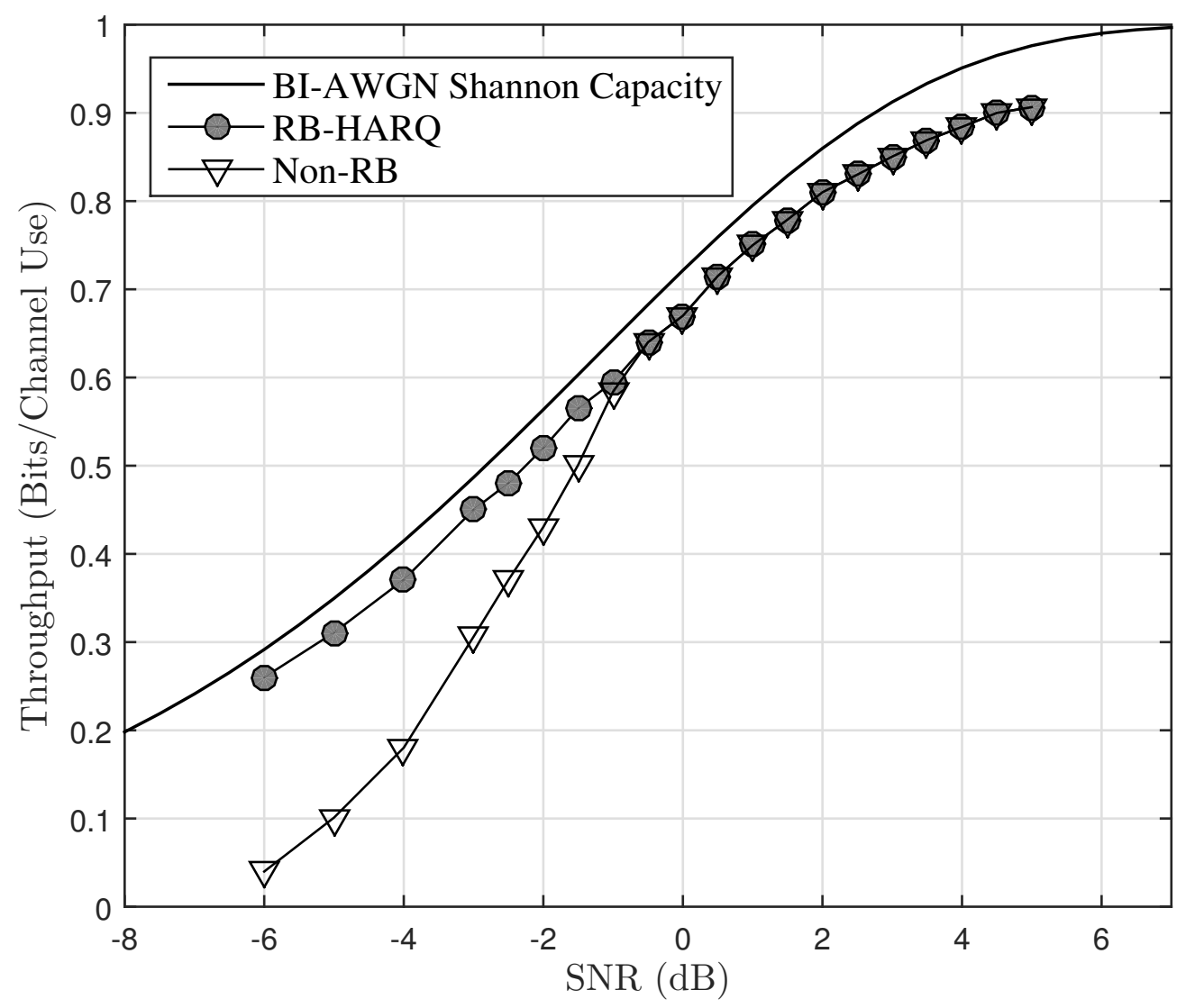

Figure 2.1: Throughput of a reliability-based IR-HARQ based on LDPC codes according to [13].

The superiority of RB-HARQ schemes over non-RB schemes comes at the expense of some complexity issues that prevent them from being used in practice. In particular, the decoder needs to send information specifying which code bits most need to be retransmitted. For example in [13] and [18] the receiver sorts the received code bits from the least to the most reliable and asks for retransmission of a number of the least reliable ones. This not only introduces complexity to the system but also affects the actual throughput due to the time needed for communication over the feedback channel, since the index of each requested code bit requires $\log _{2} N$ bits to specify, where $N$ is the block length. These issues need to be addressed before the RB-HARQ schemes can be considered for practical communications systems. In Chapter 3 we attempt to address these issues, and provide a better reliability metric. 


\subsection{LDPC Codes for IR-HARQ}

LDPC codes, first proposed by R. Gallager, are among the most promising codes in the field of channel coding [17]. Motivated by the promising performance of LDPC codes under belief propagation (BP) decoding for fixed-rate communication [21], researchers have worked on designing LDPC codes specifically for IR-HARQ. An information-theoretic analysis of some HARQ protocols, concerning throughput and the average delay for blockfading channels have been reported in [22]. For practical systems, there are mainly two methods to construct RC-LDPC codes from a mother LDPC code: puncturing and extending.

With puncturing, some of the code bits of the mother LDPC code are not transmitted over the channel. At the receiver side, these code bits are considered to have log-likelihood ratios (LLRs) of zero for the BP decoder. To ensure the rate-compatibility of such schemes, puncturing is done to get higher rate codes from a lower-rate mother code. To develop good puncturing algorithms for the LDPC codes, under BP decoding, it is useful to monitor the message passing operation used in the BP decoder operating on the Tanner graph [23]. Since the initial message outgoing from every punctured variable node is zero, i.e., erasures, it is desired to have the least number of erasures at the first iteration of the BP decoder. Therefore, heuristically one good puncturing algorithm is to puncture the variable nodes with the smallest variable degree, as proposed in [24]- [26]. However this may not necessarily be the best way to puncture LDPC codes. In these methods, the locations of the punctured bits are chosen almost arbitrarily and the resultant codes may suffer from performance loss especially at high rates due to the failure in recovering the stopping sets [27]. Tian et al. [28] proposed rate-compatible LDPC codes by puncturing lower-triangular parity-check matrices where the puncturing does not violate the degree distribution profiles of mother codes. Ha and McLaughlin [29]- [31] studied the optimal puncturing of LDPC codes in the sense which optimizes the threshold of the decoder. However the analysis is mainly based on the cycle-free asymptotic condition, i.e., an infinite block length, so the puncturing method is not necessarily effective at finite lengths. Later in [32]- [33] they studied the puncturing of finite length LDPC codes to find good puncturing patterns. They introduced

the concept of the recovery tree and the step of recoverability for each code bit and variable node in the Tanner graph of the code. They presented a two-step algorithm for puncturing the codeword in such a way that maximizes the number of code bits that have as a small step of recoverability as possible. There have been other works on puncturing of LDPC codes [34], [35]. These works are either to improve that of [33] in terms of the error-floor, 


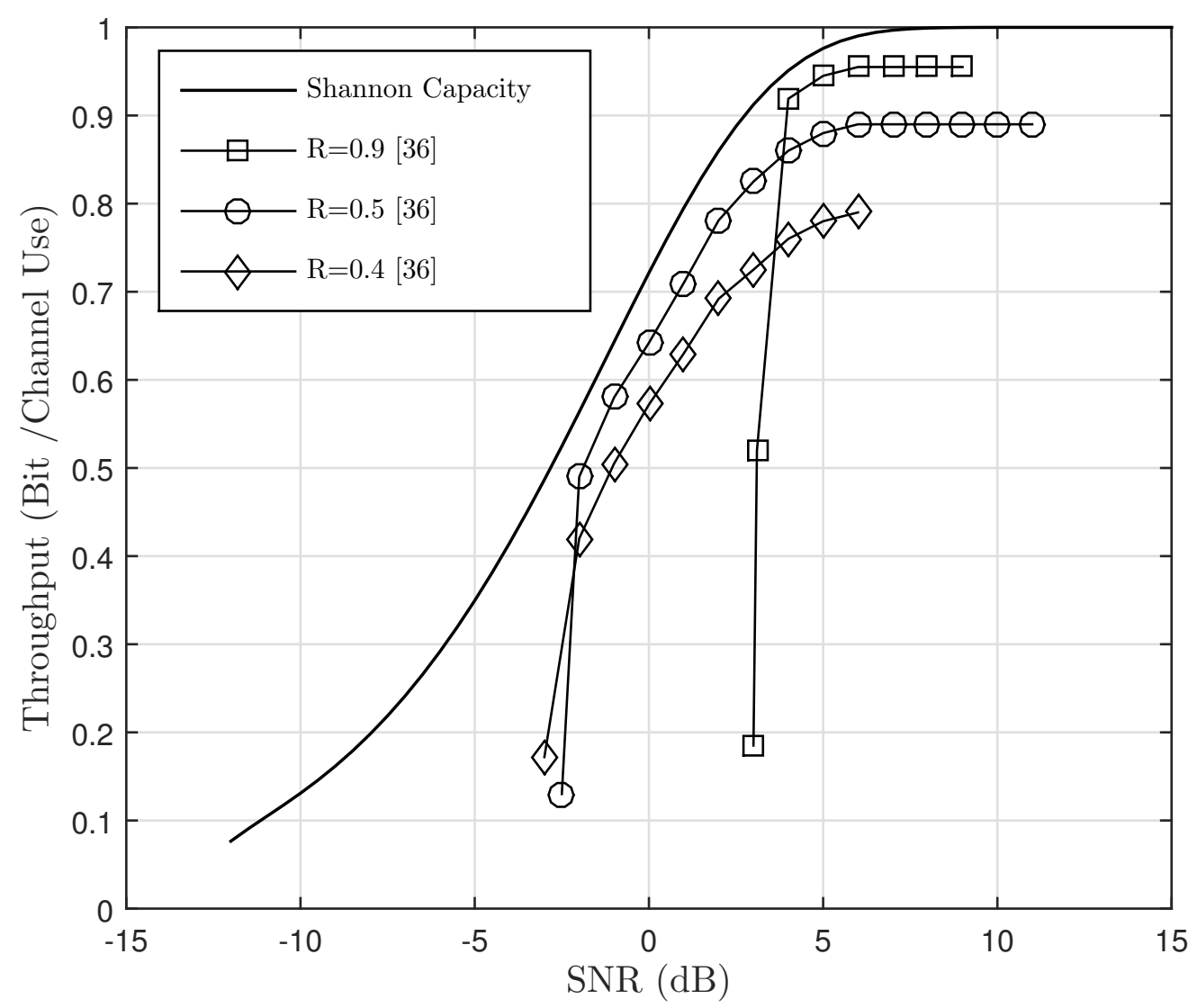

Figure 2.2: The throughput of IR-HARQ with LDPC codes using the puncturing method in [36] for different mother code rates.

which is not relevant to the throughput of the IR-HARQ system, or consider an asymptotic analysis. The authors in [36] proposed an LDPC-based hybrid ARQ scheme with random transmission assignments. That is, the puncturing pattern is randomly chosen according to a distribution. The spectrum properties of LDPC code ensembles are derived. The performance of the scheme proposed in [36] is shown in Fig. 2.2 , and as the figure suggests, random transmission does not result in capacity approaching IR-HARQ for a wide range of SNRs. As reported in [37] and observed in Fig. 2.2, it is necessary to use a low-rate mother code to get good performance at low rates (low SNRs), but puncturing is not likely to yield good high-rate codes if the rate of the mother code is too low, so that a large number of code bits need to be punctured. 


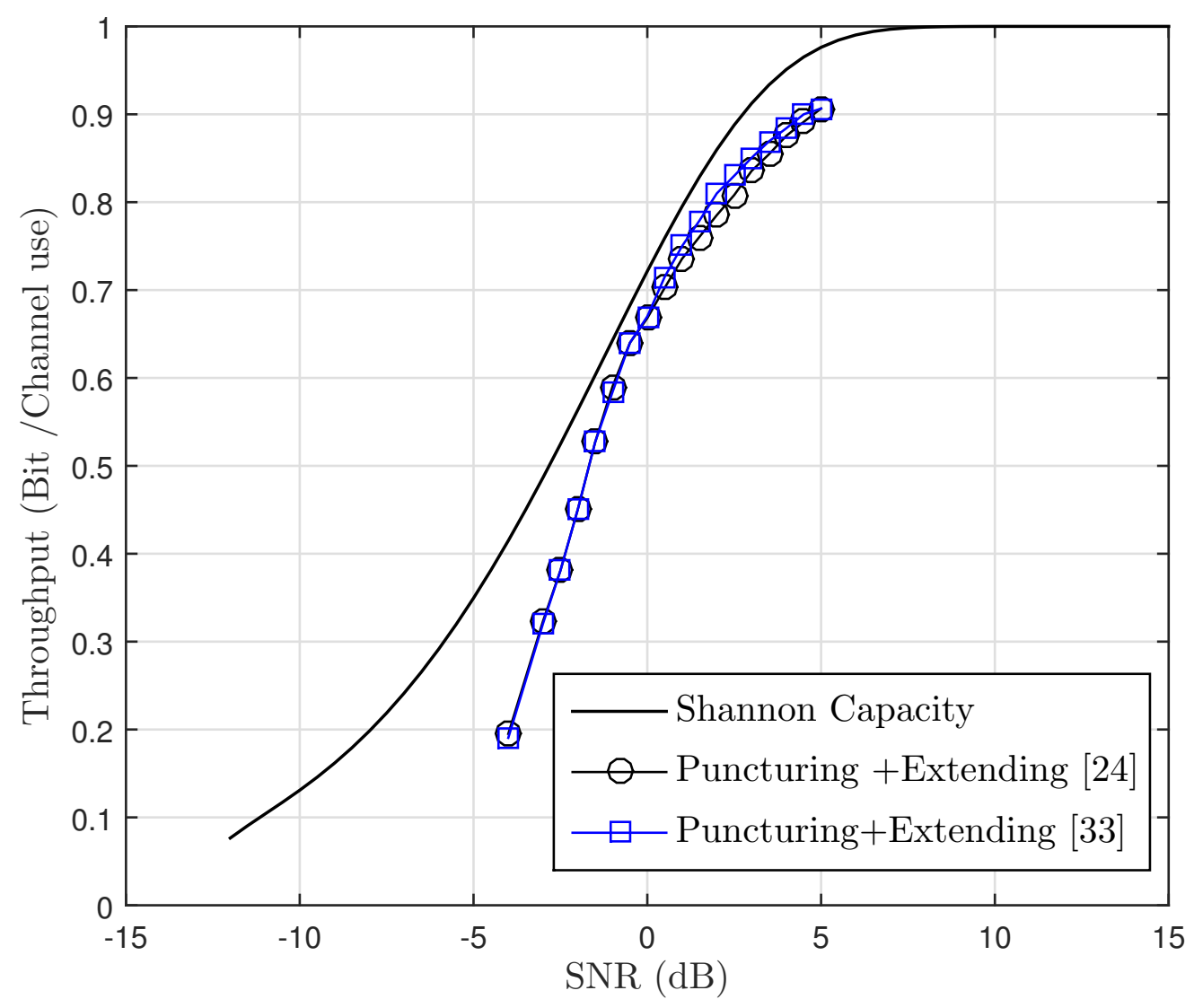

Figure 2.3: The throughput of IR-HARQ based on [24] and Ha's puncturing method [33].

The authors in [37] introduced the idea of extending the mother code and reported improvements in throughput performance. A modified version of extending using irregular progressive edge growth (PEG) [38] is studied in [24]. Although puncturing and extending have brought improvements in the throughput performance, the throughput leans away from the capacity for both high and low SNRs. In other words having a universal capacity approaching IR-HARQ scheme at all SNRs seems to be unlikely. Fig. 2.3 depicts the throughput of IR-HARQ with RC LDPC codes constructed according to [24] with Ha's puncturing method [33]. As can be seen, the throughput drops off sharply at low SNRs, and the gap to the capacity is significant.

Another method to construct RC codes for IR-HARQ scheme is based on the notion of "check splitting". With check splitting which was originally proposed in [39] and further studied in [40]- [44] a high-rate mother code is extended by adding a new parity check 
equation for a new code bit. It is known that high-rate LDPC codes need high-degree check nodes to perform well by keeping the connectivity of the Tanner graph high. Similarly, it is known that low-rate LDPC codes require low degree checks to perform well under BP decoding. Check splitting is a method that makes it possible to have a high-rate code with high check-node degree and low-rate code with low check-node degree while ensuring the rate-compatibility of the high and low rate codes. This is done by splitting a check node of high-degree into two check nodes of lower degrees. IR-HARQ schemes based on check splitting are highly efficient and increase the operating SNR range. However this comes at the price of more scheduling complexity for the BP decoder. For low rates, check splitting introduces too many degree-two variable nodes which violates the stability conditions of the parallel BP decoder. To overcome this, a more complex BP decoder can be used which requires a more complex serial scheduling which is the main drawback of the schemes based on check splitting.

\subsection{LDGM codes for IR-HARQ}

With LDPC codes, decoding is done on the Tanner graph of the parity check matrix. This is mainly because the parity check matrix of the code is sparse, as in the definition of an LDPC code, and thus will result in linear-time decoding complexity. However, for any linear block code, a similar BP decoding algorithm can operate on the Tanner graph corresponding to the generator matrix of the code. In this thesis we refer to the Tanner graphs corresponding to the parity check matrix and the generator matrix of a code as the parity check graph and the generator graph. Similarly to LDPC codes, to maintain the complexity of decoding on the generator graph of a code, the generator matrix needs to be sparse. In this thesis we refer to LDGM codes as the codes whose generator matrix is sparse. This includes but is not limited to the standard definition of the LDGM code as an LDPC code with the parity check matrix of the form $\mathbf{H}=\left[\begin{array}{ll}\mathbf{I} & \mathbf{P}\end{array}\right]$ [45]- [47]. LDGM codes have the advantage of linear time encoding and decoding as well as their capability to be extended easily by adding additional columns to their generator matrices, and thus can be well-suited for use with IR-HARQ.

LDGM codes and their BP decoding are closely related to the area of rateless codes. As examples of this class of codes, we can name LT code [48], Raptor codes [49] and Fountain codes [50], which were originally designed to achieve the capacity of the binary erasure channel (BEC) in broadcast networks. In particular, the decoding of LT codes involves 
performing BP decoding on the generator graph of the code. Although with LT codes these graphs may not be necessarily sparse, to maintain the coding complexity for practical systems sparse graphs are desired. Therefore practical LT codes should have low-density generator matrices which are the essence of LDGM codes.

As originally designed for BEC channels, LT codes can have asymptotically vanishing overhead. However this is achieved with relatively high decoding complexity. In an attempt to reduce the complexity of LT codes, Raptor codes were introduced as an extension of LT codes in the form of a serial concatenation of LT codes and a high-rate pre code (e.g., LDPC code). With this configuration, the raptor code allow linear time encoding and decoding complexity and thus are well-suited for IR-HARQ.

As we have seen, LDGM codes and their BP decoding are closely related to LT and Raptor codes. LDGM codes for IR-HARQ are mainly in the form of randomly constructed LDGM codes. Specifically, each column of the generator matrix is constructed in the following way. A degree, $d$ is sampled from a variable node degree distribution, $\lambda^{g}(x)=\sum_{i} \lambda_{i}^{g} x^{i}$, where $\lambda_{i}^{g}$ is the probability that the chosen degree is $i$. Then $d$ randomly selected message bits are chosen and their sum is considered as the new code bit [48]. Equivalently, for each column of the generator matrix, a degree, $d$, is sampled from the degree distribution and then $d$ ones are placed randomly in an otherwise all-zero column vector. Having perfect knowledge of the generator matrix, the receiver performs BP decoding on the generator graph. In Chapter 4 we presents the preliminaries of the LDGM codes and their BP decoding on the generator graphs.

The random construction of the generator matrix is shown to be effective in packet-based erasure networks, as they were the original target of the LT and Raptor codes. However, in IR-HARQ the overhead resulting from the added bits to each transmitted packet specifying which information packets are added together to obtain the transmitted packet, gets large and thus it it is necessary to eliminate this overhead by using a deterministic generator matrix known to the receiver prior to start of the data transmission. The generator matrix of the LDGM code can be constructed from a degree sequence for the variable nodes of the Tanner graph, just in the same way as LT codes work. To extend this generator matrix, a certain number of variable nodes are added to the Tanner graph and the edges are put randomly according to the chosen degree of the variable node. The performance of the resultant LDGM code mainly depends on the degree distribution $\lambda^{g}(x)$. A density evolution (DE) method based on the Gaussian approximation(GA) [51] to find good degree distributions for LDGM codes is presented in [52]. Fig. 2.4 depicts the throughput results of an 


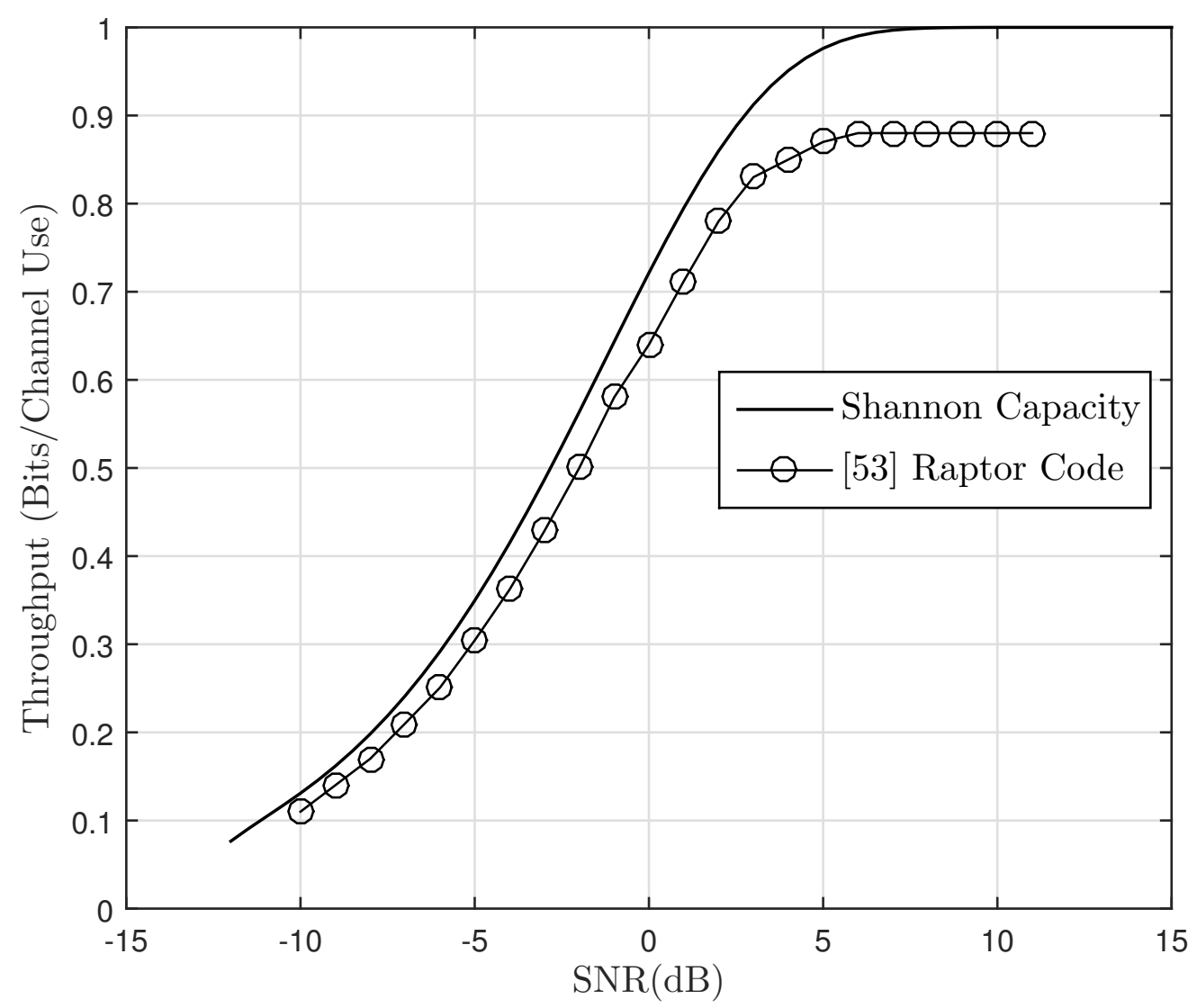

Figure 2.4: Throughput of IR-HARQ with Raptor codes.

IR-HARQ scheme based on Raptor codes [53]. As can be seen, due to the overhead of the Raptor code, there is a ceiling at high SNRs, and the gap to the capacity gets bigger as the SNR increases. It seems that unlike LDPC codes, Raptor codes, as a representative of the LDGM class of codes, are very effective at low SNRs.

\subsection{Polar Codes for IR-HARQ}

Polar codes are the first class of structured channel codes which are proved to achieve the capacity of binary input memoryless output symmetric (BIMOS) channels [54]- [57]. This ability to achieve capacity is ensured under a simple low-complexity successive cancellation (SC) decoding. However, capacity-achieving is an asymptotic attribute of polar codes. The performance analysis of polar codes for finite lengths reveals that their performance is 
worse than that of their competitors such as LDPC codes. To improve their finite-length performance, numerous, more advanced, types of decoders have been proposed [58]- [66]. However, the performance improvements brought by these decoders comes at a significant increase in the decoding complexity. Therefore for practical purposes the SC decoder would be preferred. We consider the SC decoder for polar codes for the rest of this thesis. To obtain RC-polar codes, puncturing can be used. With puncturing, zero LLRs are fed to the nodes corresponding to the punctured bits at the last column of the graphical representation of the generator matrix of the polar code referred to as the PC graph. The authors in [67] proposed a quasi-uniform puncturing (QUP) algorithm for puncturing polar codes. The QUP algorithm punctures the code bits in such a way that it tries to keep the distance between any two adjacent punctured code bits as uniform as possible, and thus the name quasi-uniform puncturing. QUP is shown to possess good properties reflecting the minimum Hamming distance of the punctured code. In particular a quantity referred to as the row weight of the generator matrix is employed to measure the Hamming distance of the code. It is shown that QUP produces punctured polar codes with good row weight, and thus good minimum Hamming distance, properties. Specifically it is shown to have a larger minimum row weight than the average row weight of random puncturing, when the code length becomes sufficiently large. There are also other puncturing algorithms for polar codes. In [68] the authors proposed a puncturing algorithm designed for use with BP decoding of polar codes [66]. Another puncturing algorithm was proposed in [69] and was reported to be effective at very short code lengths. The authors in [70] considered asymptotic rate-compatible puncturing of polar codes and showed the existence of capacity-achieving punctured polar codes for any given puncturing fraction. On the other hand, extending algorithms for polar codes have been barely addressed. In fact the only proposed extending algorithm for polar code is the trivial retransmission method [71]. In Chapter 5 we review the puncturing and extending algorithms for polar codes in detail. The authors in [71] proposed an IR-HARQ scheme based on polar codes which uses QUP puncturing and selective retransmission of the message bits. Fig. 2.5 depicts the throughput performance of that scheme. As can be seen, unlike the fixed-rate performance of the polar codes under the SC decoding, the throughput performance is close to capacity, particularly at high SNRs. 


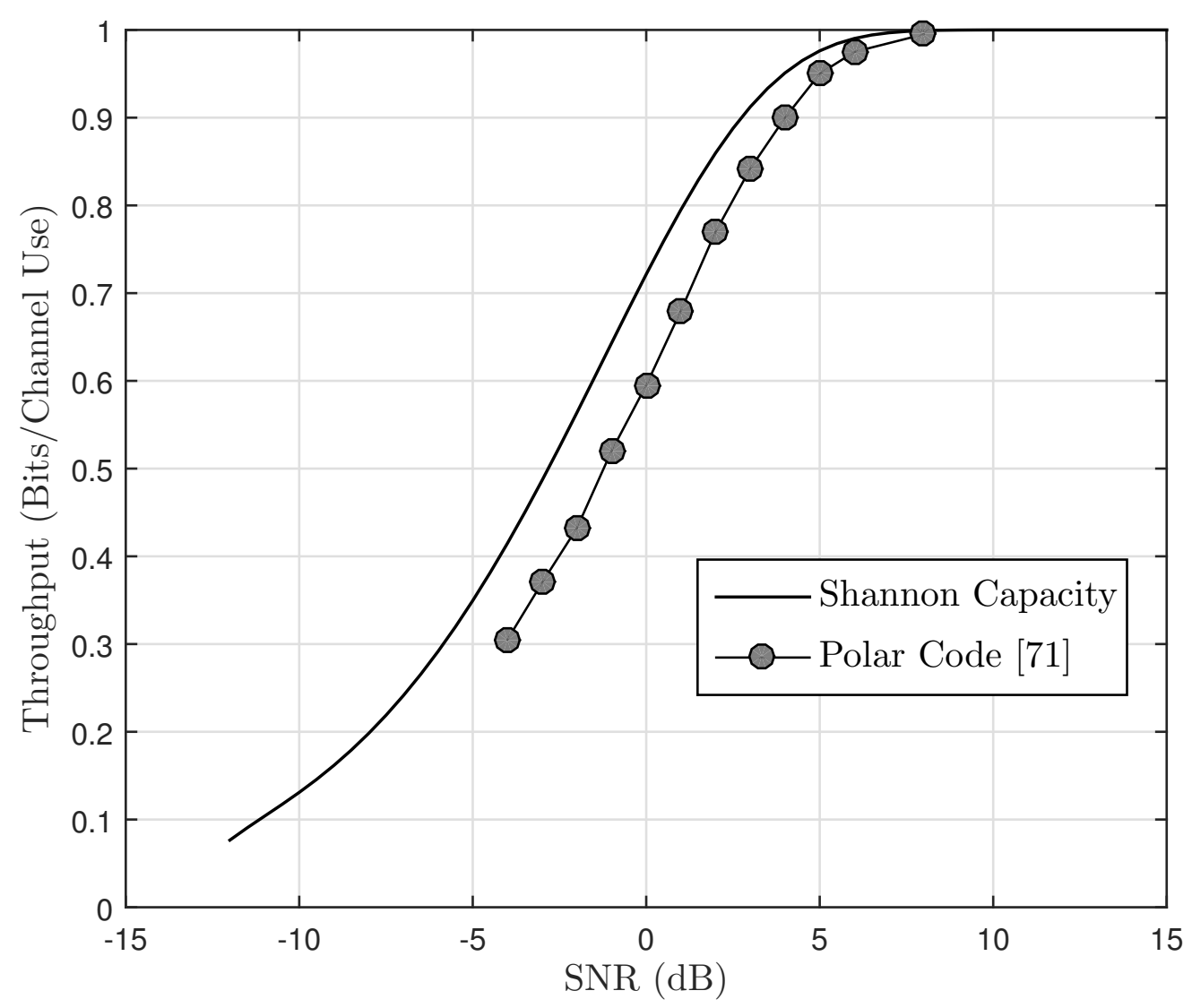

Figure 2.5: Throughput of IR-HARQ based on Polar codes constructed according to [71].

\subsection{Summary}

We summarize the advantages and disadvantages of the above IR-HARQ schemes. The performance of IR-HARQ schemes can be improved by employing reliability-based HARQ with additional overhead introduced via the feedback channel which needs to be maintained for practical systems. IR-HARQ schemes with LDPC codes can get close to the capacity at intermediate SNRs, or the SNR for which the mother code is designed. Although Ha's method for puncturing LDPC codes can result in good performance at higher SNRs, the throughput performance suffers at low SNRs where extending needs to be employed to construct lower rate codes. In other words, IR-HARQ schemes based on LDPC codes do not seem to be promising at the rates far away from the rate of the LDPC mother code. The decoding of such schemes is simple and linear with the length of the code. With LDGM codes, as a representative of the class of codes with decoding on the generator graph, the 
performance seem to be very promising at low SNRs. However as the SNR increases the gap to the capacity increases. At very high SNRs the throughput is quite far from the capacity. Furthermore, IR-HARQ schemes based on Raptor codes, will suffer from the overhead of the mother code. That is, the throughput will not go above a certain amount even at the very high SNRs. Although it is possible to mitigate this issue by making the code systematic, it would require extra nonlinear-time complexity at the encoder. With polar codes it seems that the throughput performance remains close to capacity for a wide range of SNRs. However the gap to capacity is larger than that of IR-HARQ with LDPC and LDGM codes. In the following chapters we propose new codes for IR-HARQ with the goal of maximizing throughput for as wide a range of SNR as possible. 


\section{Chapter 3}

\section{Reliability-based IR-HARQ}

\subsection{Code Structure}

The proposed RB-HARQ scheme ensures rate-compatibility via puncturing and extending of a mother code. Here we utilize the same code construction approach introduced in [24], [37]. But we make use of an optimum puncturing method for finite length LDPC codes [33] rather than the heuristic approach used in [24]. Fig. 3.1 shows the structure of the code our proposed RB-HARQ schemes uses. $\mathbf{H}_{M}$ is the parity check matrix of the mother code. The mother code is of arbitrary rate of the form $a / b$ with the parity check matrix size of $(b-a) W \times b W$ for arbitrary integers $a$ and $b, a<b . \mathbf{H}_{\mathrm{E}}$ is referred to as the extension matrix. We allow the extension matrix, $\mathbf{H}_{\mathrm{E}}$, to be of arbitrary size $W \times W$. The degree distribution of LDPC codes play an important role in their asymptotic analysis [21]. We denote the node-perspective variable node degree distribution of the LDPC code by $\lambda^{\prime}(x)=\sum_{i} \lambda_{i}^{\prime} x^{i}$, where $\lambda_{i}^{\prime}$ is the fraction of variable nodes with degree $i$. In order to have a powerful mother code to be used in our RB-HARQ scheme, the mother code should be constructed with a degree distribution which has a threshold as close as possible to the Shannon capacity. We use the following degree distribution found in [38] and proposed in [24] to be used in rate-compatible LDPC code construction.

$$
\begin{aligned}
\lambda^{\prime}(x)= & 0.47532 x^{2}+0.2795 x^{3}+0.0348 x^{4} \\
& +0.1088 x^{5}+0.1013 x^{15}
\end{aligned}
$$

For a code rate of $1 / 2$, and via density evolution we found that the above degree distribution has a threshold of $-2.43 \mathrm{~dB}$ for binary-input additive white Gaussian noise (BI-AWGN) 
channel. This threshold is almost $0.4 \mathrm{~dB}$ away from Shannon limit. We construct $\mathbf{H}_{M}$ according to the above degree distribution. We have used progressive edge growth (PEG) algorithm to construct $\mathbf{H}_{M}$ [38]. To get higher rate codes, puncturing is done on the mother code. The puncturing method for finite length LDPC codes proposed in [33], which is based on the concept of the recovery tree of a punctured variable node, is used here.

To get lower rate codes, the mother code's parity-check matrix is extended by adding a number of columns to its right end and an equal number of rows to its bottom. We refer to the matrix $\mathbf{H}_{E}$ as the extension matrix. Similar to the parity check matrix of the mother code, $\mathbf{H}_{M}$, the extension matrix is constructed via the PEG algorithm with the same degree distribution in (3.1). The identity matrices are used to provide correlation between the previously transmitted code bits and those of the extended codes. According to the above construction method, the $l$-extended code is of rate $a / b+l$ with a parity check matrix of size $(b-a+l) W \times(b+l) W$. The reader is referred to [37] and [24] for more details on this code structure. Although the mother code could theoretically be extended indefinitely, it is more practical to limit the maximum number of extensions to some finite value, $N_{E}$, so the maximum codeword length is $N=\left(b+N_{E}\right) W$. In [24], the parameters $W=128, a=5$, $b=13$ and $N_{E}=6$ were used.

In the proposed incremental redundancy scheme the transmission is carried out in clusters of $S$ bits, where $S$ is a divisor of $W$. There are a total of $N / S$ clusters. In the puncturing phase the same approach as in [24] is used, i.e., code bits are transmitted according to the puncturing pattern in clusters of $S$ bits. This process is repeated until all the clusters of the mother code have been transmitted. If decoding still fails the next phase starts. In this phase reliability metrics are used to transmit the code bits of the extended codes. The first cluster of the first extended code is transmitted and decoding is attempted on the Tanner graph of the first extended code. If decoding fails, the receiver calculates a "reliability metric" for each cluster in the extended code. These reliability metrics are based on the code bit log-likelihood ratios (LLRs) after decoding, and are calculated according to one of the algorithms described in Section III. The least reliable cluster is selected for transmission. This cluster may have been previously transmitted, in which case maximum ratio combining is used to combine the multiple received samples, or it may be a new cluster. After transmission of the requested cluster, decoding on the graph of the extended code is again attempted, and this process is repeated until decoding succeeds, or $\mathrm{W} / \mathrm{S}$ clusters have been transmitted. If decoding still fails, the transmission of the parity bits of the next lower rate code commences, decoding is attempted on the Tanner graph of the 


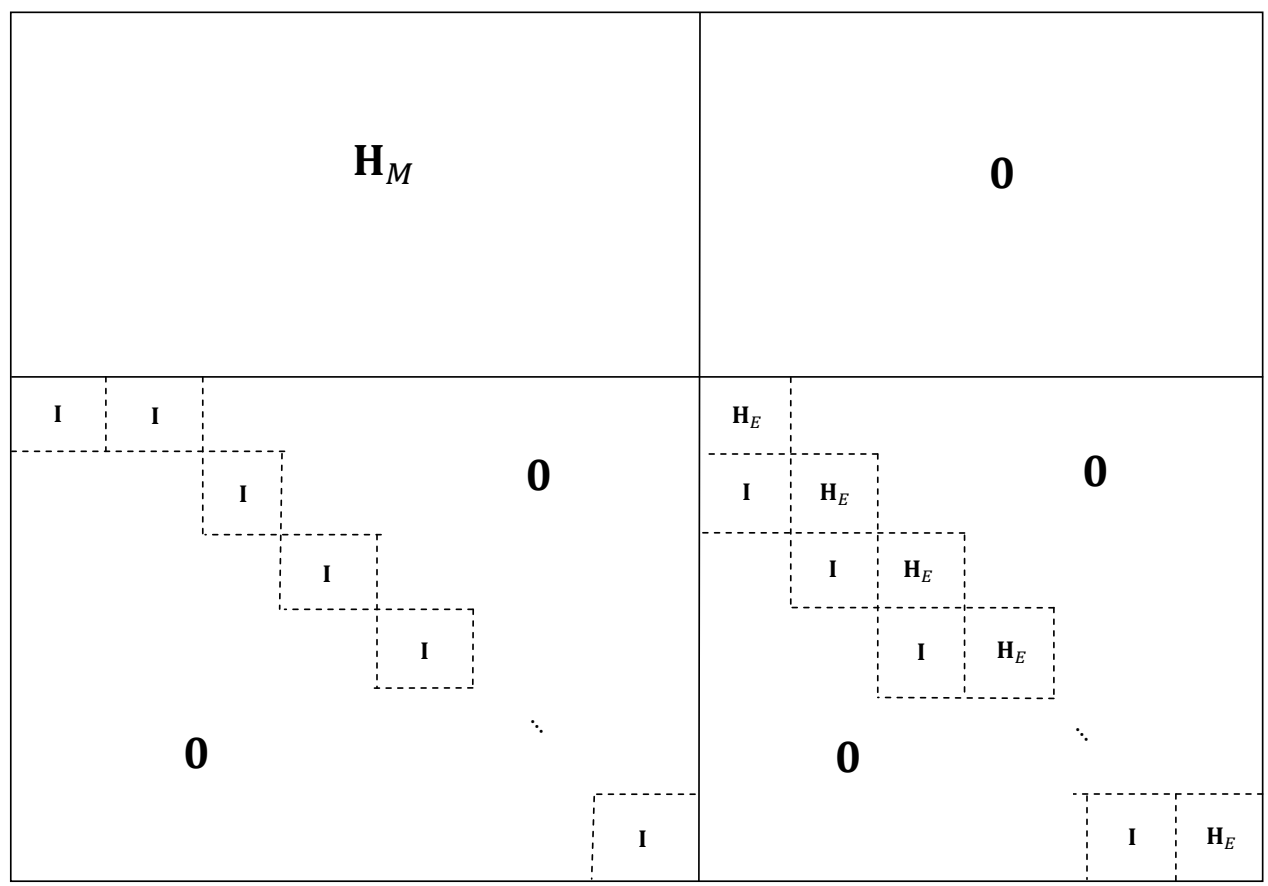

Figure 3.1: Parity check matrix structure of the extended codes.

corresponding extended code and the same procedure as the previous extended code is taken. In case of decoding failure when the longest code has been used, all decodings are attempted on the full Tanner graph.

\subsection{Reliability Metrics}

The best candidate clusters for retransmission are chosen according to one of the reliability metrics derived analytically in this section. We want to select the cluster whose retransmission will most help the decoding effort. Generally speaking we look for the cluster that is received with the least reliability. Suppose the $S$ transmitted code bits for the $k$-th cluster are denoted by $\mathbf{u}_{k}=\left[u_{k, 1}, \ldots, u_{k, S}\right]$. For the purpose of this discussion, assume that the elements of cluster $\mathbf{u}_{k}$ are i.i.d. and take values from $\{ \pm 1\}$ equiprobably. Although this assumption is not true in general, the resulting reliability metrics are nonetheless effective. For the metric derivations we consider the transmission of $\mathbf{u}_{k}$ over a BI-AWGN channel, so the received cluster $\mathbf{r}_{k}=\left[r_{k, 1}, \ldots, r_{k, S}\right]$ can be written as $\mathbf{r}_{k}=\mathbf{u}_{k}+\mathbf{n}_{k}$ where $\mathbf{n}_{k}=\left[n_{k, 1}, \ldots, n_{k, S}\right]$ is the i.i.d. AWGN noise vector with variance $\sigma^{2}=N_{0} / 2$. Define the 
LLR of the received sample $i$ as $L_{k, i}=\frac{2 r_{k, i}}{\sigma^{2}}$.

To assess the reliability of $\mathbf{r}_{k}$, we note that a maximum a posteriori (MAP) detector would detect $\mathbf{u}_{k}$ as

$$
\widehat{\mathbf{u}}_{k}=\arg \max _{\mathbf{u}_{k}} \operatorname{Pr}\left(\mathbf{u}_{k} \mid \mathbf{r}_{k}\right) .
$$

We refer to $\operatorname{Pr}\left(\mathbf{u}_{k} \mid \mathbf{r}_{k}\right)$ as the probability distribution of the cluster $k$. Now the question is what values of vector $\mathbf{r}_{k}$ make it less reliable. This question would matter when we have transmitted several clusters of the codeword and need to choose one of them for retransmission based on their corresponding received vectors. Intuitively, the desired distribution of $\operatorname{Pr}\left(\mathbf{u}_{k} \mid \mathbf{r}_{k}\right)$ is the one that makes a strong decision, i.e., has a high probability weight for $\widehat{\mathbf{u}}_{k}$ and small weights for other vectors. Ideally $\operatorname{Pr}\left(\mathbf{u}_{k} \mid \mathbf{r}_{k}\right)=\delta\left[\mathbf{u}_{k}-\widehat{\mathbf{u}}_{k}\right]$ where $\delta[n]$ is the Kronecker delta function. We define the likelihood ratio of a vector $\widehat{\mathbf{u}}_{k}$ as

$$
L\left(\widehat{\mathbf{u}}_{k}, \mathbf{u}_{k}, \mathbf{r}_{k}\right)=\frac{\operatorname{Pr}\left(\widehat{\mathbf{u}}_{k} \mid \mathbf{r}_{k}\right)}{\operatorname{Pr}\left(\mathbf{u}_{k} \mid \mathbf{r}_{k}\right)}
$$

For a given $\mathbf{r}_{k}$ to yield a strong and reliable decision, the function $L\left(\widehat{\mathbf{u}}_{k}, \mathbf{u}_{k}, \mathbf{r}_{k}\right)$ should have the property that its value for any $\mathbf{u}_{k} \neq \widehat{\mathbf{u}}_{k}$ is much bigger than for $\mathbf{u}_{k}=\widehat{\mathbf{u}}_{k}$. Note that the function has a minimum at $\mathbf{u}_{k}=\widehat{\mathbf{u}}_{k}$ at which its value is equal to 1 . We make use of this desired feature of the function $L\left(\widehat{\mathbf{u}}_{k}, \mathbf{u}_{k}, \mathbf{r}_{k}\right)$ to reflect the strength of the decision made according to it. In the following we present three ways in which making a strong decision is prevented and thus a reliability metric can be obtained to determine the best cluster candidates for retransmission.

\subsubsection{Metric A}

One case that prevents a strong decision is when all the $\mathbf{u}_{k}$ have an LLR as close to unity. This can happen when the maximum of this function is pushed to its minimum. Then the least reliable cluster, denoted by $\tilde{k}$, can be defined as

$$
\tilde{k}=\arg \min _{k} \max _{\mathbf{u}_{k} \neq \widehat{\mathbf{u}}_{k}} L\left(\widehat{\mathbf{u}}_{k}, \mathbf{u}_{k}, \mathbf{r}_{k}\right) .
$$

We have

$$
\operatorname{Pr}\left(\mathbf{u}_{k} \mid \mathbf{r}_{k}\right)=\frac{f_{\mathbf{R}_{k}}\left(\mathbf{r}_{k} \mid \mathbf{u}_{k}\right) 2^{-S}}{f_{\mathbf{R}_{k}}\left(\mathbf{r}_{k}\right)} \propto f_{\mathbf{R}_{k}}\left(\mathbf{r}_{k} \mid \mathbf{u}_{k}\right) .
$$


Since

$$
f_{\mathbf{R}_{k}}\left(\mathbf{r}_{k} \mid \mathbf{u}_{k}\right)=\frac{1}{(\sigma \sqrt{2 \pi})^{S}} \exp \left(-\frac{1}{2 \sigma^{2}}\left\|\mathbf{r}_{k}-\mathbf{u}_{k}\right\|^{2}\right)
$$

it gives

$$
\begin{aligned}
\operatorname{Pr}\left(\mathbf{u}_{k} \mid \mathbf{r}_{k}\right) & \propto \exp \left(-\frac{1}{2 \sigma^{2}}\left\|\mathbf{r}_{k}-\mathbf{u}_{k}\right\|^{2}\right) \\
& \propto \exp \left(\frac{1}{\sigma^{2}} \sum_{i=1}^{S} r_{k, i} u_{k, i}\right)
\end{aligned}
$$

So

$$
L\left(\widehat{\mathbf{u}}_{k}, \mathbf{u}_{k}, \mathbf{r}_{k}\right)=\exp \left(\frac{1}{\sigma^{2}} \sum_{i=1}^{S} r_{k, i}\left(\hat{u}_{k, i}-u_{k, i}\right)\right) .
$$

Note that $\hat{u}_{k, i}=\operatorname{sgn}\left(r_{k, i}\right)$ where sgn is the signum function, so

$$
L\left(\widehat{\mathbf{u}}_{k}, \mathbf{u}_{k}, \mathbf{r}_{k}\right)=\exp \left(\frac{1}{\sigma^{2}} \sum_{i=1}^{S}\left(\left|r_{k, i}\right|-r_{k, i} u_{k, i}\right)\right)
$$

which can be maximized by setting $\mathbf{u}_{k}=-\operatorname{sgn}\left(\mathbf{r}_{k}\right)$. Therefore the least reliable received vector is

$$
\tilde{k}=\arg \min _{k} \sum_{i=1}^{S}\left|L_{k, i}\right| .
$$

Thus with this metric, the least reliable cluster is the one with the smallest sum of the absolute LLRs. This makes sense as we know that the bits with the smallest LLR values are associated with less reliability. In particular an LLR value of zero indicates that the decision on the value of the bit is completely random with maximum uncertainty.

\subsubsection{Metric B}

Preventing a strong decision can also happen when the LLR of one of the $\mathbf{u}_{k} \mathrm{~s}$ gets as close to the minimum of the function, rather than requiring the LLR of all the $\mathbf{u}_{k}$ to be small simultaneously. In this case the $\mathbf{u}_{k}$ which has the second smallest LLR is required to have an LLR as close as possible to that of $\widehat{\mathbf{u}}_{k}$. The least reliable cluster is then 


$$
\tilde{k}=\arg \min _{k} \min _{\mathbf{u}_{k} \neq \widehat{\mathbf{u}}_{k}} L\left(\widehat{\mathbf{u}}_{k}, \mathbf{u}_{k}, \mathbf{r}_{k}\right) .
$$

To minimize (4.16), $\sum_{i=1}^{S} r_{k, i} u_{k, i}$ needs to be maximized with the constraint $\mathbf{u}_{k} \neq \widehat{\mathbf{u}}_{k}$. This linear optimization has the solution

$$
u_{k, i}= \begin{cases}\operatorname{sgn}\left(r_{k, i}\right), & i \neq m_{k} \\ -\operatorname{sgn}\left(r_{k, i}\right), & i=m_{k}\end{cases}
$$

where $m_{k}=\arg \min _{i}\left|r_{k, i}\right|$. Therefore

$$
\min _{\mathbf{u}_{k} \neq \widetilde{\mathbf{u}}_{k}} L\left(\widehat{\mathbf{u}}_{k}, \mathbf{u}_{k}, \mathbf{r}_{k}\right) \propto \exp \left(\frac{2\left|r_{k, m_{k}}\right|}{\sigma^{2}}\right)
$$

With this result, the least reliable received vector $\mathbf{r}_{k}$ is

$$
\tilde{k}=\arg \min _{k} \min _{i \in\{1, \ldots, S\}}\left|L_{k, i}\right| .
$$

Thus with this metric, the least reliable cluster is the one with the smallest absolute LLR.

\subsubsection{Metric C}

Another case that results in strong decision is when $\widehat{\mathbf{u}}_{k}$ has a large value of $\operatorname{Pr}\left(\mathbf{u}_{k}=\hat{\mathbf{u}}_{k} \mid \mathbf{r}_{k}\right)$. This causes the LLR of the $\mathbf{u}_{k}$ to get far away from 1. From an information theoretical point of view this is equivalent to measure the distance of the distribution $\operatorname{Pr}\left(\mathbf{u}_{k} \mid \mathbf{r}_{k}\right)$ to the Kronecker delta $\delta\left[\mathbf{u}_{k}-\widehat{\mathbf{u}}_{k}\right]$ via the KL divergence. Define $D\left(\mathbf{r}_{k}\right)$ as

$$
D\left(\mathbf{r}_{k}\right)=D\left(\delta\left[\mathbf{u}_{k}-\widehat{\mathbf{u}}_{k}\right] \| \operatorname{Pr}\left(\mathbf{u}_{k} \mid \mathbf{r}_{k}\right)\right)=-\log \left(\operatorname{Pr}\left(\widehat{\mathbf{u}}_{k} \mid \mathbf{r}_{k}\right)\right) .
$$

Then the least reliable cluster is the one whose $\mathbf{r}_{k}$ maximizes $D\left(\mathbf{r}_{k}\right)$. Since 


$$
\begin{aligned}
\operatorname{Pr}\left(\widehat{\mathbf{u}}_{k} \mid \mathbf{r}_{k}\right) & \propto \frac{\exp \left(-\frac{1}{2 \sigma^{2}}\left\|\mathbf{r}_{k}-\widehat{\mathbf{u}}_{k}\right\|^{2}\right)}{\sum_{\mathbf{u}_{k}} \exp \left(-\frac{1}{2 \sigma^{2}}\left\|\mathbf{r}_{k}-\mathbf{u}_{k}\right\|^{2}\right)} \\
& =\frac{\exp \left(-\frac{1}{2 \sigma^{2}}\left[\left\|\mathbf{r}_{k}\right\|^{2}+S-2 \sum_{i}\left|r_{k, i}\right|\right]\right)}{\sum_{\mathbf{u}_{k}} \exp \left(-\frac{1}{2 \sigma^{2}}\left[\left\|\mathbf{r}_{k}\right\|^{2}+S-2 \mathbf{r}_{k}^{T} \mathbf{u}_{k}\right]\right)} \\
& =\frac{\exp \left(\sum_{i} \frac{\left|r_{, i, i}\right|}{\sigma^{2}}\right)}{\sum_{\mathbf{u}_{k}} \exp \left(\frac{\mathbf{r}_{k}^{T} \mathbf{u}_{k}}{\sigma^{2}}\right)} \\
& =\frac{\exp \left(\sum_{i} \frac{\left|r_{k, i}\right|}{\sigma^{2}}\right)}{\prod_{i}\left[\exp \left(\frac{\left|r_{k, i}\right|}{\sigma^{2}}\right)+\exp \left(\frac{-\left|r_{k, i}\right|}{\sigma^{2}}\right)\right]} \\
& =\prod\left[1+\exp \left(\frac{-2\left|r_{k, i}\right|}{\sigma^{2}}\right)\right]^{-1}
\end{aligned}
$$

the least reliable cluster is

$$
\tilde{k}=\arg \max _{k} \prod_{i}\left(1+\exp \left(-\left|L_{k, i}\right|\right)\right) .
$$

We therefore propose to use the above reliability metrics, but using the LLRs of the code bits after decoding in place of $L_{k, i}$. Note that in case of measuring the reliability of bits rather than clusters (i.e. when $S=1$ ), all three metrics are equivalent, and reduce to the one used in [13].

\subsection{Simulation Results}

We consider transmission over the AWGN channel as well as a quasi-static flat Rayleigh fading channel with bipolar binary phase shift keying (BPSK) signaling. The code structure parameters are as follows. To have a fair comparison with the published work [24], the rate of the mother code is chosen as 8/13 with $a=8, b=13$ and $W=256$. We have used a total of $N_{E}=12$ extensions. In case of decoding failure after transmitting the last extended code, all decoding attempts are done on the Tanner graph of the lowest-rate code. The throughput is defined as the number of bits in a message frame divided by the average number of code bits required for successful decoding.

Fig. 3.2 shows the throughput of the system for each of the three different reliability metrics, 


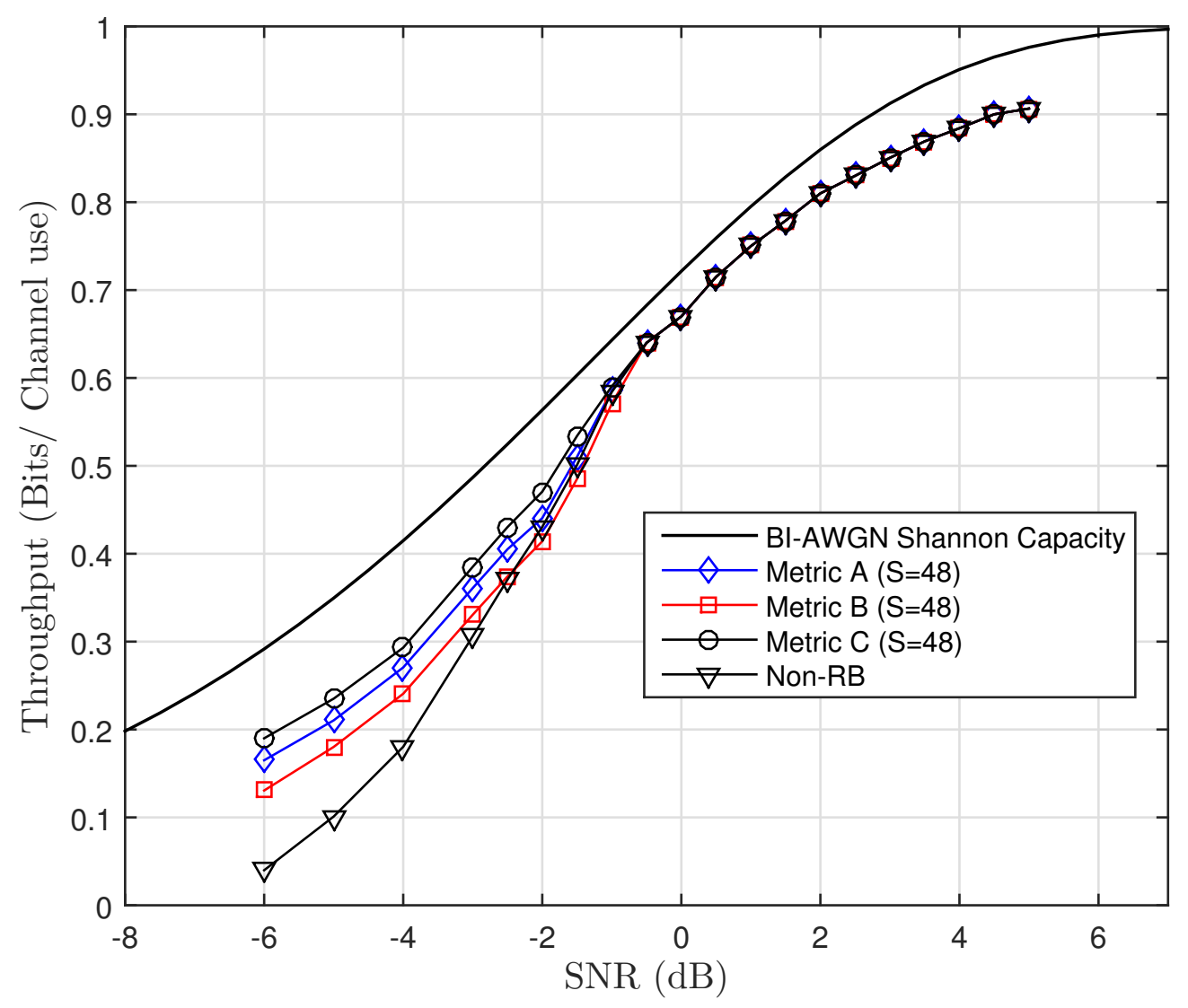

Figure 3.2: Throughput of the proposed RB-HARQ system with the three different reliability metrics.

using a cluster size of $S=48$, along with the throughput of a traditional system that does not use reliability feedback (labelled "Non-RB"). The non-RB scheme uses the same mother code as in [24], punctured with Ha's algorithm [33], but allows for up to $N_{E}=50$ extensions to avoid problems when the fully extended code is still not long enough for successful decoding. As can be seen, use of reliability feedback with any of the metrics provides better performance than the non-RB scheme, particularly at low signal-to-noise rations (SNRs). As also shown in Fig. 3.2, the metric $C$ outperforms the other two. We will therefore focus on this metric for the remainder of this work.

One important parameter of the feedback scheme is the cluster size, $S$. By using a smaller cluster size it is possible to more precisely specify which code bits should be transmitted, 


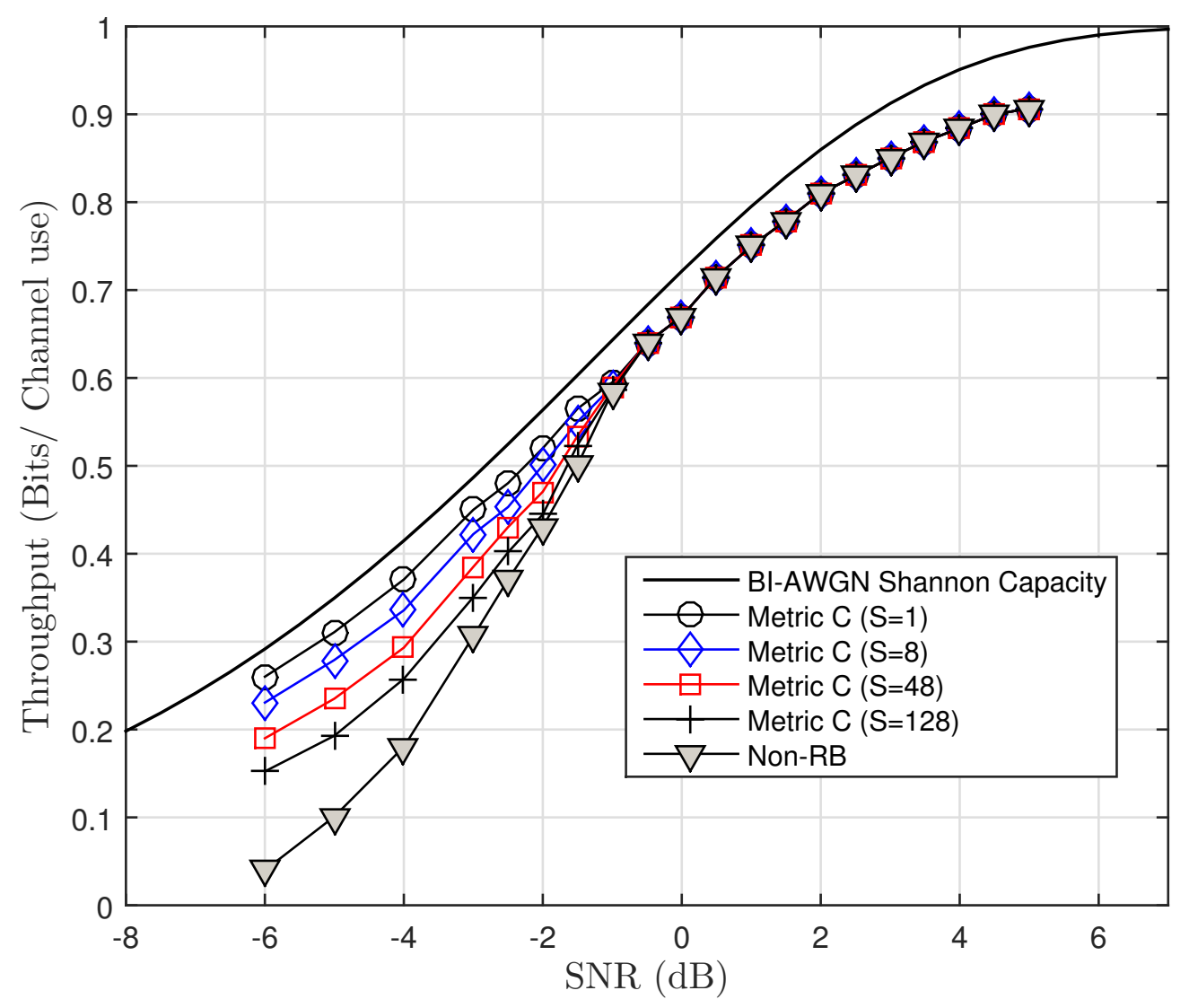

Figure 3.3: The effect of the cluster size on the throughput of the proposed RB-HARQ system.

thereby reducing the number of unnecessary code bit retransmissions, which in turn improves throughput, as shown in Fig. 3.3. We can see the advantage of using a very small ( $S=1)$ cluster size over small $(S=8)$, intermediate $(S=48)$, and large $(S=128)$ cluster sizes. However, there is a penalty with using small sizes because more bits are needed to specify the cluster, thereby increasing the amount of the feedback required. The number of bits needed to specify a cluster of size $S$ within a codeword of length $N$ is $\log _{2}(N / S)$, which should be kept small.

It is possible to adjust the throughput, taking into account the feedback. For each cluster which has been transmitted in the feedback mode of the system, the actual number of transmitted bits is $S+\log _{2}(N / S)$, while for all the other clusters this quantity is equal to $S$. 


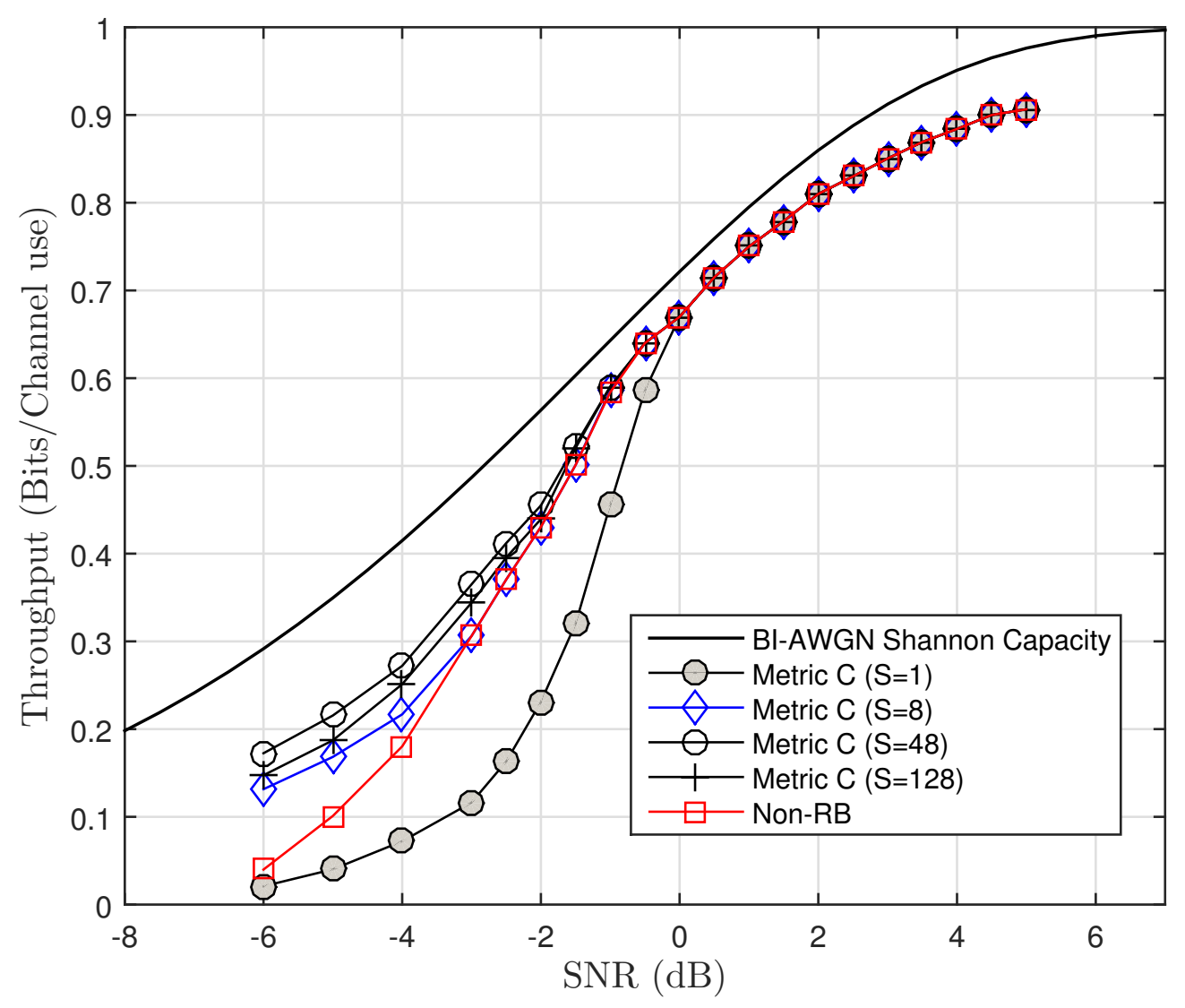

Figure 3.4: Throughput after compensating for the feedback overhead.

Fig. 3.4 shows the throughput results of the proposed method with different cluster sizes, taking into account the feedback overhead according to the above. As it can be seen even with feedback overhead taken into account, there is still up to $2 \mathrm{~dB}$ gain over the nonfeedback method, provided that the cluster size is selected appropriately. A cluster size of $S=48$ is the most efficient, which has a corresponding feedback overhead of $14 \%$. Note that metric C with a cluster size of $S=1$ is used in [13] and [18], and as can be seen, when compensated for the feedback overhead, results in significant drop-off in throughput as it requires a large overhead. 


\subsection{Conclusions}

The design of RB-HARQ with LDPC codes based on feedback is considered. The proposed scheme measures the reliability of clusters of code bits instead of individual code bits. By requesting retransmission of whole clusters, along with partial use of the feedback channel in the extended code mode, the proposed scheme is significantly less complex for practical systems compared to the previous RB-HARQ systems. The analytical derivation of different reliability metrics to choose the best candidate cluster are presented. Our simulation results show that an efficient reliability metric can result in up to $2 \mathrm{~dB}$ gain over the previous RB-HARQ schemes as well as schemes which do not use reliability metrics. 


\section{Chapter 4}

\section{Combined LDPC and LDGM Codes for IR-HARQ}

\subsection{Introduction}

As mentioned before, LDPC and LDGM codes have both been employed with IR-HARQ. In this chapter we consider designing an IR-HARQ scheme based on both of these codes together. We use the BP decoder in [52] for decoding the LDGM code on the generator matrix graph. The advantage of LDGM codes over LDPC codes is the reduction in encoding complexity. The IR-HARQ schemes that use the RC-LDGM codes show good performance at low SNRs, but suffer from the overhead problem which causes the throughput saturation at high SNRs. It is noteworthy that there are ways to overcome the throughput saturation by making the code systematic as proposed in [52] but this comes with a superfluous polynomial time encoding complexity. The throughput of IR-HARQ schemes based on LDGM codes, is significantly far from the Shannon capacity at high SNRs, while being promisingly capacity approaching at low SNRs.

To construct RC codes which could result in IR-HARQ schemes that perform close to the capacity for all SNRs, our general idea is to build a sequence of RC codes, $\left\{C_{n}\right\}$, where the nature of the codes varies from higher rates to lower rates. By nature we mean one of the two natures: LDPC and LDGM. Since the throughput of IR-HARQ schemes based on LDGM codes are weak at high SNRs we would like code $C_{n}$ to be close to the family of LDPC codes when $C_{n}$ has a high rate. Similarly, we would like lower-rate codes to be close to the family of LDGM codes to improve the throughput performance at low SNRs. To accomplish this goal, we design an LDPC mother code of fairly high rate using the PEG algorithm with an optimized degree distribution. This code is then punctured to higher 
rates, resulting in RC-LDPC codes of high rates. To have lower-rate codes be close to the LDGM codes, we propose to extend the generator matrix of the LDPC mother code by adding extending columns to it. We show how to find a generator matrix of the LDPC code that is good for extending. The extending columns are constructed according to an optimized degree distribution for the LDGM code of certain rates. As we construct lowerrate codes by extending the generator matrix of the LDPC code, the codes become more close to the family of LDGM codes.

Having constructed the RC code sequence, $\left\{C_{n}\right\}$, along with their parity check and generator graphs, with varying nature from LDPC codes to the LDGM codes, we show how this varying nature can be employed to develop a decoding protocol with a soft transition behaviour from decoding on the parity check graph to the generator graph, as the rate of $C_{n}$ decreases. In particular, we propose a combined decoding which operates on both the generator and parity check graphs and show how it can improve the throughput performance at the intermediate SNRs. To facilitate this, we also show how to construct the parity check matrices of the extended codes from their generator matrices, in such a way that is suitable for performing decoding on the parity check graph. We also show how the receiver determines whether or not to decode $C_{n}$ on its parity check graph, generator graph or to perform the combined decoding on both, depending on the rate of $C_{n}$.

The rest of this chapter is organized as follows. In Section 4.2 we present preliminaries of the LDGM codes and their belief propagation (BP) decoding. In Section 4.3 we present the proposed method to construct the sequence of RC-LDPC/LDGM codes. In Section 4.4, we explore different decoding methods for the RC-LDPC/LDGM codes. Particularly, decoding of the codes on their Tanner graphs corresponding to generator or parity check matrices is proposed and a combined decoding method is developed to work on both graphs. Section 4.5 presents the simulation results of the proposed IR-HARQ scheme. Section 4.6 concludes the chapter.

\subsection{LDGM Codes}

In this section we present some preliminaries of LDGM codes, which are linear block codes whose generator matrix is sparse, i.e., has many fewer ones than zeros. Similarly to LDPC codes it is possible to construct a Tanner graph corresponding to the generator matrix of the LDGM code. We refer to this graph as the generator graph. Consider an LDGM code with message word length $K$ and codeword length $N$. Let $\mathbf{G}=\left[g_{i, j}\right]$ be the 


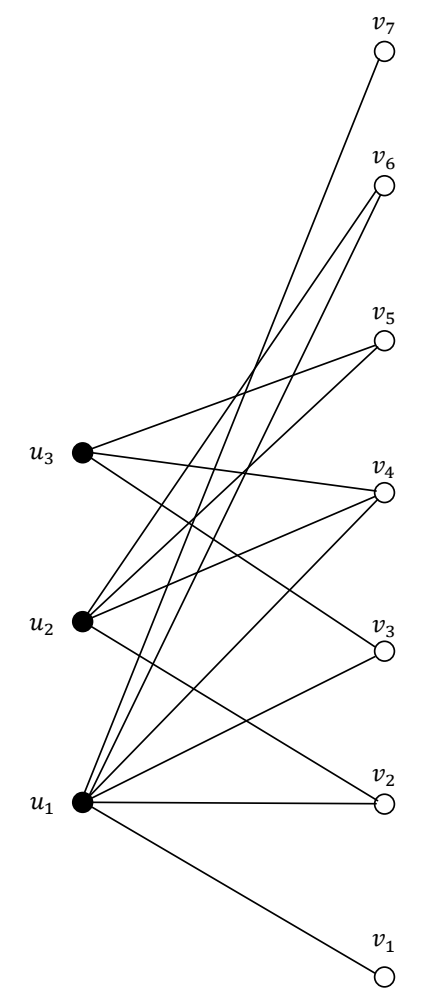

Figure 4.1: Generator graph of a sample LDGM code.

generator matrix of this code. The generator graph of the LDGM code is then a bipartite graph with two sets of nodes, the information nodes $\mathcal{U}=\left\{u_{1}, u_{2}, \ldots, u_{K}\right\}$ and the variable nodes $\mathcal{V}=\left\{v_{1}, v_{2}, \ldots, v_{N}\right\}$. Information node $u_{i}$ is connected to $v_{j}$ if and only if $g_{i, j}=1$. Fig. 4.1 depicts the generator graph corresponding to a sample LDGM code with

$$
\mathbf{G}=\left[\begin{array}{lllllll}
1 & 1 & 1 & 1 & 0 & 1 & 1 \\
0 & 1 & 0 & 1 & 1 & 1 & 0 \\
0 & 0 & 1 & 1 & 1 & 0 & 0
\end{array}\right]
$$

Encoding of a message word is simply done by applying the information bits to the information nodes of the generator graph. The value of each variable node is then equal to the summation of its neighboring information nodes. The BP decoder operates by iteratively passing messages along the edges of the generator graph. Unlike the BP decoder for LDPC codes for which the messages are estimators of the value of the variable nodes, 
the messages of the LDGM BP decoder are estimators of the value of the corresponding information nodes. Let $m_{u v}^{(l)}$ and $m_{v u}^{(l)}$ be the messages passed from information node $u$ to variable node $v$ and vice versa, at iteration number $l$. Also let $r_{v}$ be the channel output of variable node $v$ and $m_{v}^{(0)}=\log \frac{\operatorname{Pr}\left(v=0 \mid r_{v}\right)}{\operatorname{Pr}\left(v=1 \mid r_{v}\right)}$ be the log-likelihood ratio (LLR) of the a posteriori probabilities (APPs) of variable node $v$. For example, in the case of binary phase shift keying (BPSK) with mapping $0 \rightarrow+1,1 \rightarrow-1$, over the additive white Gaussian noise (AWGN) channel with one-sided noise power spectral density $N_{0}$, we have $m_{v}^{(0)}=\frac{4 r_{v}}{N_{0}}$. Let $\mathcal{U}_{v}$ denote the set of information nodes connected to variable node $v$. Similarly let $\mathcal{V}_{u}$ be the set of variable nodes connected to information node $u$. The BP decoder then iterates as follows [52]

$$
\begin{array}{ll}
m_{v u}^{(l)}=2 \tanh ^{-1}\left(\tanh \frac{m_{v}^{(0)}}{2} \prod_{u^{\prime} \in \mathcal{U}_{v} \backslash u} \tanh \frac{m_{u^{\prime} v}^{(l-1)}}{2}\right), & l \geq 1 \\
m_{u v}^{(l)}=\sum_{v^{\prime} \in \mathcal{V}_{u} \backslash v} m_{v^{\prime} u}^{(l)}, & l \geq 1
\end{array}
$$

where $\backslash$ denotes the set difference operator. The decoding starts by the information nodes sending out zero messages to all neighbor variable nodes, i.e., $m_{u v}^{(0)}=0 \forall u$ and $v \in \mathcal{V}_{u}$. Note that the above iteration updates hold true for any binary input memoryless output symmetric channel. As an important difference with LDPC codes, the LDGM BP decoder needs at least one degree-one variable node to start off the decoding process, or else all messages will remain zero through the iterations.

The number of edges connected to a node in the generator graph is referred to as the degree of that node. Let $\lambda_{i}^{g}$ be the fraction of variable nodes with degree $i$. The variable node degree distribution, $\lambda^{g}(x)$, is then defined as $\lambda^{g}(x)=\sum_{i} \lambda_{i}^{g} x^{i}$. The performance of LDGM codes under BP decoding highly depends on the degree distribution of the code [52]. Good degree distributions can be found by density evolution [52].

\subsection{Proposed RC LDPC/LDGM Code Construction}

Here we present a novel method to construct RC codes for use with IR-HARQ schemes. For a given message word length, $K$, we construct a sequence of RC codes, $\left\{C_{n}\right\}$, with rates $R_{n}=K / n$. Generally speaking, we would like the codes with rates higher than $R_{h}=K / N_{h}$ to be chosen from the family of LDPC codes and codes with rates lower than $R_{h}$ to be 
chosen from the family of LDGM codes, for some properly chosen $R_{h}$.

To construct RC codes, we begin by designing an LDPC mother code of rate $R_{h}$. The variable node degree distribution, $\lambda^{h}(x)=\sum_{i} \lambda_{i}^{h} x^{i}$, of the mother code is determined using density evolution in such a way that the threshold of the BP decoder is maximized [21]. Then a parity check matrix of size $\left(N_{h}-K\right) \times N_{h}$ is constructed with this degree distribution according to the PEG algorithm [38]. This parity check matrix defines the mother code, $C_{N_{h}}$, of rate $R_{h}$.

To construct higher rate codes, we use a puncturing algorithm to puncture this code. One of the simplest, and yet most effective, methods to puncture a finite length LDPC code is the method presented in [33]. We use this algorithm to puncture the code $C_{N_{h}}$ and obtain the codes $C_{n}, n=N_{h}-1, \ldots, K$. Having done this we have constructed the RC codes $C_{n}$, $n=K, \ldots, N_{h}$. These codes are from the class of punctured LDPC codes.

Lower-rate codes are constructed by extending the generator matrix of the mother code. To do so it is first necessary to find a good generator matrix $\mathbf{G}_{N_{h}}$ for the parity check matrix $\mathbf{H}_{N_{h}}$ of the LDPC mother code, which can be done using the algorithm we propose in Section 4.3.1. The extending procedure is given in Section 4.3.2. To ensure that the extended codes provide good performance, they are designed for an optimized LDGM degree distribution, $\lambda^{g}(x)$, at a target rate $R_{g} \leq R_{h}$. Similarly to LDPC codes, density evolution is used to determine the degree distribution [52].

\subsubsection{Construction of the generator matrix $\mathbf{G}_{N_{h}}$}

We first find a generator matrix, $\mathbf{G}_{N_{h}}$, of the LDPC $\operatorname{code} C_{N_{h}}$ with parity check matrix, $\mathbf{H}_{N_{h}}$. Let $\mathbf{G}_{N_{h}}=\left[\begin{array}{ll}\mathbf{G}_{s} & \mathbf{G}_{p}\end{array}\right]$ be a generator matrix of $\mathbf{H}_{N_{h}}$, where $\mathbf{G}_{s}$ and $\mathbf{G}_{p}$ are of sizes $K \times K$ and $K \times\left(N_{h}-K\right)$, respectively. Also, without loss of generality, assume $\mathbf{H}_{N_{h}}=\left[\begin{array}{ll}\mathbf{H}_{s} & \mathbf{H}_{p}\end{array}\right]$, where $\mathbf{H}_{s}$ and $\mathbf{H}_{p}$ are of sizes $\left(N_{h}-K\right) \times K$ and $\left(N_{h}-K\right) \times\left(N_{h}-K\right)$, respectively, and $\mathbf{H}_{p}$ is full-rank. If $\mathbf{G}_{N_{h}}$ is a generator matrix of $\mathbf{H}_{N_{h}}$ then

$$
\mathbf{G}_{N_{h}} \mathbf{H}_{N_{h}}^{T}=\mathbf{0}
$$

where $\mathbf{0}$ is a $K \times\left(N_{h}-K\right)$ all-zero matrix. Therefore

$$
\left[\begin{array}{ll}
\mathbf{G}_{s} & \mathbf{G}_{p}
\end{array}\right]\left[\begin{array}{ll}
\mathbf{H}_{s} & \mathbf{H}_{p}
\end{array}\right]^{T}=\mathbf{0}
$$


so

$$
\mathbf{G}_{s} \mathbf{H}_{s}^{T}+\mathbf{G}_{p} \mathbf{H}_{p}^{T}=\mathbf{0}
$$

or

$$
\mathbf{G}_{s} \mathbf{H}_{s}^{T}=\mathbf{G}_{p} \mathbf{H}_{p}^{T}
$$

This implies

$$
\mathbf{G}_{p}=\mathbf{G}_{s} \mathbf{H}_{s}^{T} \mathbf{H}_{p}^{-T}
$$

so

$$
\mathbf{G}_{N_{h}}=\left[\begin{array}{ll}
\mathbf{G}_{s} & \mathbf{G}_{s} \mathbf{H}_{s}^{T} \mathbf{H}_{p}^{-T}
\end{array}\right] .
$$

For $\mathbf{G}_{N_{h}}$ to be a generator matrix of $\mathbf{H}_{N_{h}}$ it also needs to be full-rank. Suppose $\mathbf{y}$ is in the range (column space) of $\mathbf{G}_{N_{h}}$, i.e.

$$
\mathbf{y}=\mathbf{G}_{N_{h}} \mathbf{x}
$$

for some binary column vector $\mathbf{x}$ of length $N_{h}$. Then

$$
\mathbf{y}=\left[\begin{array}{ll}
\mathbf{G}_{s} & \mathbf{G}_{s} \mathbf{H}_{s}^{T} \mathbf{H}_{p}^{-T}
\end{array}\right]\left[\begin{array}{c}
\mathbf{x}_{1} \\
\mathbf{x}_{2}
\end{array}\right]
$$

where $\mathbf{x}_{1}$ and $\mathbf{x}_{2}$ are column vectors containing the first $K$ and the last $N_{h}-K$ elements of $\mathbf{x}$, respectively. So

$$
\begin{aligned}
\mathbf{y} & =\mathbf{G}_{s} \mathbf{x}_{1}+\mathbf{G}_{s} \mathbf{H}_{s}^{T} \mathbf{H}_{p}^{-T} \mathbf{x}_{2} \\
& =\mathbf{G}_{s} \mathbf{x}_{1}+\mathbf{G}_{s} \mathbf{x}_{3}
\end{aligned}
$$

where $\mathbf{x}_{3}=\mathbf{H}_{s}^{T} \mathbf{H}_{p}^{-T} \mathbf{x}_{2}$. So

$$
\mathbf{y}=\mathbf{G}_{s}\left(\mathbf{x}_{1}+\mathbf{x}_{3}\right)=\mathbf{G}_{s} \mathbf{x}^{\prime}
$$

for some $\mathbf{x}^{\prime}=\mathbf{x}_{1}+\mathbf{x}_{3}$. Eq. (4.13) shows that $\mathbf{y}$ is also in the range of $\mathbf{G}_{s}$, and therefore the rank of $\mathbf{G}_{N_{h}}$ is less than or equal to the rank of $\mathbf{G}_{s}$. Since we require $\mathbf{G}_{N_{h}}$ to be full-rank then $\mathbf{G}_{s}$ must also be full-rank, i.e. of rank $K$. On the other hand if $\mathbf{G}_{s}$ is full-rank then so 
is $\mathbf{G}_{N_{h}}$ as it contains a $K \times K$ full-rank matrix $\mathbf{G}_{s}$. So (4.9) yields a generator matrix for $\mathbf{H}_{N_{h}}$, if and only if the arbitrarily chosen matrix $\mathbf{G}_{s}$ is full-rank. For any full-rank choice of $\mathbf{G}_{s},(4.9)$ gives a generator matrix.

Since there are $\prod_{i=0}^{K-1}\left(2^{K}-2^{i}\right)$ possible full-rank choices for $\mathbf{G}_{s}$, there are that many different generator matrices for $\mathbf{H}_{N_{h}}$. Among these matrices we are interested in those which have the desired degree distribution, $\lambda^{g}(x)$. We therefore take $K$ samples of this degree distribution to obtain the degree sequence $d_{1}, \ldots, d_{K}$. This degree sequence is then given to the PEG algorithm to construct a $K \times K$ Tanner graph corresponding to $\mathbf{G}_{s}$. However to ensure that this matrix is full-rank we employ a modified version of the PEG algorithm which only considers matrices that are upper-triangular with the main diagonal elements being 1 . Obviously for this PEG algorithm to work, the degree sequence $\left\{d_{i}\right\}$ should be sorted in ascending order, i.e. $d_{i} \leq d_{j}$ for $i<j$. Then, given $\mathbf{G}_{s}, \mathbf{G}_{N_{h}}$ can be calculated by (4.9).

\subsubsection{Construction of the extended generator matrices, $\mathbf{G}_{n}$}

Having constructed a suitable generator matrix $\mathbf{G}_{N_{h}}$ for the code $C_{N_{h}}$, we can construct $\left\{C_{n}\right\}$ for $n=N_{h}+1, N_{h}+2, \ldots$, recursively. Let $\mathbf{G}_{n-1}$ be the generator matrix of the code $C_{n-1}$. We construct code $C_{n}$ by extending code $C_{n-1}$, i.e. by adding one column to $\mathbf{G}_{n-1}$

$$
\mathbf{G}_{n}=\left[\begin{array}{ll}
\mathbf{G}_{n-1} & \mathbf{g}_{n}
\end{array}\right]
$$

where $\mathbf{g}_{n}$ is referred to as an extending column. The Hamming weight, $d_{n}$, of this column is chosen by sampling the degree distribution $\lambda^{g}(x)$, and then placing $d_{n}$ ones in an otherwise all-zero $K \times 1$ vector, resulting in $\mathbf{g}_{n}$. Equivalently, the Tanner graph of $\mathbf{G}_{n}$ is constructed by that of $\mathbf{G}_{n-1}$. Recall that the Tanner graph of $\mathbf{G}_{n-1}$ has $K$ information nodes and $n-1$ variable nodes. Using (4.14), to build the Tanner graph of $\mathbf{G}_{n}$, one variable node and $d_{n}$ edges are added to the graph. These edges emanate from the newly added variable node and are connected to information nodes determined by the PEG algorithm. These information nodes correspond to the position of the $1 \mathrm{~s}$ in the extending vector $\mathbf{g}_{n}$. With successive use of (4.14) all codes $\left\{C_{n}\right\}$ and their generator matrices are constructed. 


\subsection{Decoding of RC LDPC/LDGM Codes}

In this section we explore different approaches for decoding the RC codes $\left\{C_{n}\right\}$. Recall that we have constructed the codes $\left\{C_{n}\right\}$ with the goal of changing their nature from LDPC to LDGM starting from the rate $R_{h}=K / N_{h}$. Therefore one can propose a basic approach to decode the code $C_{n}$ on its parity check graph if $n \leq N_{h}$ and on its generator graph if $n>N_{h}$. Although this decoding approach is simple it is possible to achieve superior performance by decoding $C_{n}$ for $n>N_{h}$ on its parity check graph even though it is of LDGM nature.

\subsubsection{Parity-Check Decoding of the LDGM Codes $C_{n}, n>N_{h}$}

As said before, the $\operatorname{codes} C_{n}$ start to change their nature from LDPC to LDGM at $n=N_{h}+1$. However this change is not abrupt. For $C_{n}, n>N_{h}$, there are $N_{h}-K$ columns of $\mathbf{G}_{n}$ which are imposed by $\mathbf{G}_{p}$ in (4.8). Since these columns are not chosen according to the desired degree distribution $\lambda^{g}(x), \mathbf{G}_{n}$ is relatively far away from the desired degree distribution for values of $n$ slightly larger than $N_{h}$. As $n$ gets larger, more extending columns chosen from $\lambda^{g}(x)$ are used and $C_{n}$ becomes closer to LDGM in nature. Furthermore for $n$ slightly larger than $N_{h}$, as we will see, it is possible to find parity check matrices for $C_{n}$ which still can benefit from the desired parity check matrix $\mathbf{H}_{N_{h}}$. So even though the codes $C_{n}, n>N_{h}$ are of LDGM nature, it may be useful to decode them on their parity check graphs. In the following we show how to find good parity check matrices of these codes.

Remember that the code $C_{n}, n \geq N_{h}+1$ is constructed by extending the generator matrix using (4.14). We have

$$
\mathbf{G}_{n}=\left[\begin{array}{ll}
\mathbf{G}_{n-1} & \mathbf{g}_{n}
\end{array}\right] .
$$

Let $\mathbf{H}_{n}$ be a parity check matrix of this code. For low decoding complexity we are interested in those $\mathbf{H}_{n}$ which can be written in the form of

$$
\mathbf{H}_{n}=\left[\begin{array}{cc}
\mathbf{H}_{n-1} & \mathbf{a} \\
\mathbf{h}_{n} & c
\end{array}\right],
$$

where $c$ is a scalar, $\mathbf{a}$ is a column vector of length $n-K-1$ and $\mathbf{h}_{n}$ is a row vector of length $n-1$. For $\mathbf{H}_{n}$ to be a parity check matrix of $\mathbf{G}_{n}$ we must have

$$
\mathbf{G}_{n} \mathbf{H}_{n}^{T}=\mathbf{0},
$$


or

$$
\left[\begin{array}{ll}
\mathbf{G}_{n-1} & \mathbf{g}_{n}
\end{array}\right]\left[\begin{array}{cc}
\mathbf{H}_{n-1} & \mathbf{a} \\
\mathbf{h}_{n} & c
\end{array}\right]^{T}=\mathbf{0}
$$

which requires that

$$
\mathbf{G}_{n-1} \mathbf{H}_{n-1}^{T}+\mathbf{g}_{n} \mathbf{a}^{T}=\mathbf{0}
$$

and

$$
\mathbf{G}_{n-1} \mathbf{h}_{n}^{T}+\mathbf{g}_{n} c=0 .
$$

The first term in (4.19) is zero because $\mathbf{G}_{n-1}$ is a generator matrix of $\mathbf{H}_{n-1}$, and since $\mathbf{g}_{n}$ is not an all-zero vector we have $\mathbf{a}=\mathbf{0}$.

Now suppose that $c=0$ in (4.20). This results in $\mathbf{G}_{n-1} \mathbf{h}_{n}^{T}=\mathbf{0}$ which means that $\mathbf{h}_{n}^{T}$ is in the null space of $\mathbf{G}_{n-1}$ or equivalently, $\mathbf{h}_{n}$ is in the row space of $\mathbf{H}_{n-1}$. Therefore $\mathbf{h}_{n}$ can be written as a linear combination of the rows of $\mathbf{H}_{n-1}$. Since $\mathbf{a}$ and $c$ are already zero, this implies that the rank of $\mathbf{H}_{n}$ is still $n-K-1$ not $n-K$, i.e. it is not full-rank. So we must have $c=1$.

It is only left to find the vector $\mathbf{h}_{n}$. We begin by finding one such vector, say $\mathbf{h}_{n}^{(0)}$. From (4.20) we have

$$
\mathbf{G}_{n-1}\left(\mathbf{h}_{n}^{(0)}\right)^{T}=\mathbf{g}_{n}
$$

Now in (4.21) assume that $\mathbf{h}_{n}^{(0)}=\left[\begin{array}{ll}\mathbf{b}_{s} & \mathbf{0}\end{array}\right]$, where $\mathbf{b}_{s}$ is of size $1 \times K$. So (4.21) is reduced to

$$
\mathbf{G}_{s} \mathbf{b}_{s}^{T}=\mathbf{g}_{n}
$$

so

$$
\mathbf{b}_{s}=\mathbf{g}_{n}^{T} \mathbf{G}_{s}^{-T},
$$

where $\mathbf{G}_{s}$ is the submatrix of $\mathbf{G}_{n}$ containing its first $K$ columns. So

$$
\mathbf{h}_{n}^{(0)}=\left[\begin{array}{ll}
\mathbf{g}_{n}^{T} \mathbf{G}_{s}^{-T} & \mathbf{0}
\end{array}\right] .
$$


Now let $\mathbf{x}^{T}$ be an arbitrary vector in the null space of $\mathbf{G}_{n-1}$, then we have $\mathbf{G}_{n-1} \mathbf{x}^{T}=\mathbf{0}$. So $\mathbf{x}+\mathbf{h}_{n}^{(0)}$ is also a solution to $\mathbf{G}_{n-1} \mathbf{x}^{T}=\mathbf{g}_{n}$. Since $\mathbf{x}^{T}$ is in the null space of $\mathbf{G}_{n-1}, \mathbf{x}$ can be written as a linear combinations of the rows of $\mathbf{H}_{n-1}$. Let $\mathbf{h}_{n-1}^{(i)}$ be the $i$-th row of $\mathbf{H}_{n-1}$. Then the general solution for $\mathbf{h}_{n}$ is

$$
\mathbf{h}_{n}=\mathbf{h}_{n}^{(0)}+\sum_{i=1}^{n-K-1} \alpha_{i} \mathbf{h}_{n-1}^{(i)}
$$

for any choice of $\alpha_{i} \in \mathrm{GF}(2), i=1, \ldots, n-K-1$.

Eq. (4.25) gives $2^{n-K-1}$ solutions for $\mathbf{H}_{n}$, each corresponding to one specific choice of $\boldsymbol{\alpha}=\left[\alpha_{1}, \ldots, \alpha_{n-K-1}\right]$. The optimal solution for $\boldsymbol{\alpha}$ is the one which gives the lowest block error rate (BLER) if $C_{n}$ decoded on $\mathbf{H}_{n}$. The size of the search space for this optimization problem is $2^{n-K-1}$ which grows exponentially with the code length $n$, so this problem is hard to solve. Moreover, the evaluation of the BLER for each choice of $\boldsymbol{\alpha}$ is not straightforward. Therefore we take a different approach to choose $\alpha$ such that the decoding complexity is reduced as much as possible. Since the decoding complexity introduced by $\mathbf{h}_{n}$ is proportional to the number of ones in it, we would like to choose $\alpha$ such that the Hamming weight of $\mathbf{h}_{n}$ is minimized.

To minimize the weight of $\mathbf{h}_{n}$ we note that the term $\sum_{i=1}^{n-K-1} \alpha_{i} \mathbf{h}_{n-1}^{(i)}$ is a linear combination of the rows of $\mathbf{H}_{n-1}$, and so is a codeword of a hypothetical linear block code, $\widetilde{C}_{n-1}$, whose generator and parity check matrices are $\mathbf{H}_{n-1}$ and $\mathbf{G}_{n-1}$, respectively. Let $\mathbf{v}$ denote this codeword, so $\mathbf{h}_{n}^{(0)}=\mathbf{v}+\mathbf{h}_{n}$. One can think of this equation as a hypothetical communication scenario: The codeword $\mathbf{v}$ of $\widetilde{C}_{n-1}$ has been transmitted over a BSC channel, an error pattern of $\mathbf{h}_{n}$ has occurred and $\mathbf{h}_{n}^{(0)}$ has been received at the receiver. If one can find the codeword $\mathbf{v}$ with minimum Hamming distance to the received word $\mathbf{h}_{n}^{(0)}$, the resultant error pattern, $\mathbf{h}_{n}$, will be of minimum Hamming weight. Therefore, one could employ an ML decoder for $\widetilde{C}_{n-1}$, decode the received vector $\mathbf{h}_{n}^{(0)}$, to obtain the decoded codeword $\widehat{\mathbf{v}}$, and then choose $\mathbf{h}_{n}=\mathbf{h}_{n}^{(0)}+\widehat{\mathbf{v}}$. Since it is computationally infeasible to perform ML decoding for $\widetilde{C}_{n-1}$, we resort to suboptimum decoders such as the BP decoder instead. Therefore we propose the following method to choose $\mathbf{h}_{n}$ : We perform BP decoding of $\widetilde{C}_{n-1}$ for a received word of $\mathbf{h}_{n}^{(0)}$ over a BSC channel with crossover probability $\epsilon$. We perform LDGM BP decoding of $\widetilde{C}_{n-1}$ on its generator graph, $\mathbf{H}_{n-1}$, and LDPC BP decoding of $\widetilde{C}_{n-1}$ on its parity check graph, $\mathbf{G}_{n-1}$. If any of these decodings succeeds, we choose $\mathbf{h}_{n}=\mathbf{h}_{n}^{(0)}+\hat{\mathbf{v}}$, where $\hat{\mathbf{v}}$ is the decoded codeword, otherwise we choose $\mathbf{h}_{n}$ as $\mathbf{h}_{n}^{(0)}$. The crossover probability of the BSC channel should be chosen appropriately such that decoding success is achieved. Note that 


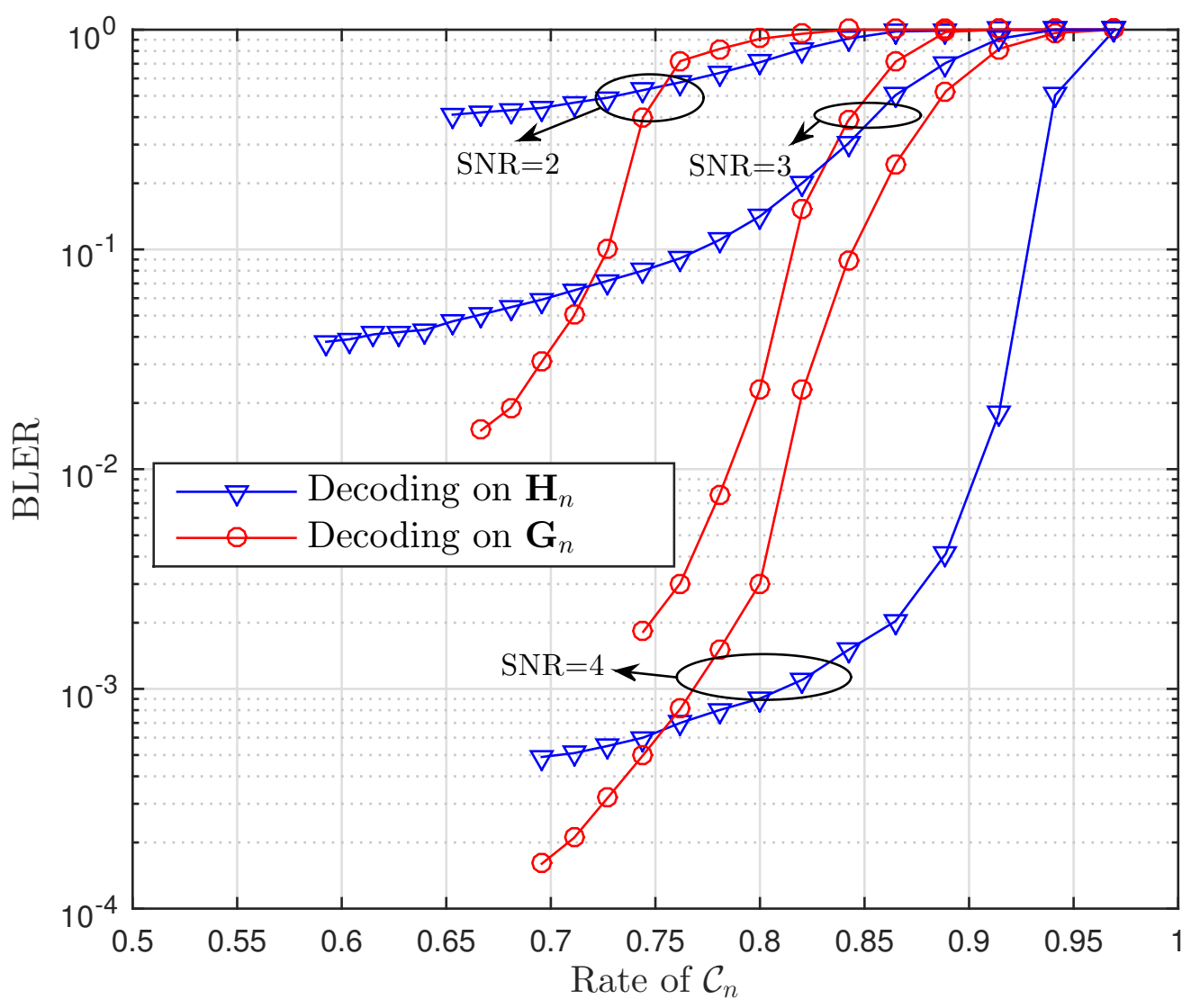

Figure 4.2: BLER of the two decoding methods for the code sequence $\left\{C_{n}\right\}$ in terms of their corresponding rates.

for a BSC channel of crossover probability the channel LLRs used for the BP decoders are calculated as $\log \frac{1-\epsilon}{\epsilon}$ for a zero received bit and $-\log \frac{1-\epsilon}{\epsilon}$ for a one.

Having constructed the parity check matrices $\mathbf{H}_{n}, n>N_{h}$, it is possible to improve the decoding performance of $C_{n}$. We can evaluate the BLER performance of $C_{n}$ under two types of decoding: Decoding on the parity check graph $\mathbf{H}_{n}$, and decoding on the generator graph $\mathbf{G}_{n}$. It is expected that for $n$ smaller than a certain value, $n_{0}$, (i.e. at high rates), decoding on $\mathbf{H}_{n}$ will be superior to decoding on $\mathbf{G}_{n}$ while the converse will be true for $n>n_{0}$, (i.e., at low rates). Fig. 4.2 depicts the BLER of a sequence of constructed codes under these two types of decoding over a binary-input AWGN (BI-AWGN) channel with BPSK. The mother code rate is $R_{h}=0.93$, and $K=4096$. The threshold values, $n_{0}$, can be determined by the crossover rates, $R_{0}$, in the graph. For example, at an SNR of $3 \mathrm{~dB}$, the 
crossover rate is around .83 , so $n_{0}$ is around $K / .83$, while at an SNR of $4 \mathrm{~dB}$ the crossover threshold is $K / .76$. Having determined $n_{0}$ at each SNR, the decoder should decode on $\mathbf{H}_{n}$ for $n<n_{0}$ and decode on $\mathbf{G}_{n}$ otherwise. As can be seen, even though the code of rate .85 is of an LDGM nature, at a SNR of $3 \mathrm{~dB}$ is is better to decode it on $\mathbf{H}_{n}$ than $\mathbf{G}_{n}$. With this decoding method it is possible to achieve the minimum of the two curves.

\subsubsection{Combined Decoding}

We have seen that it is possible to decode the code $C_{n}$ on either of its two Tanner graphs: The generator graph or the parity check graph. At any given SNR, one of these two graphs will result in a smaller BLER, so it is preferred to perform the decoding on that graph. However, for any specific channel realization, it is possible that decoding will fail on one graph but succeed on the other. Therefore, it is even better to attempt decoding on both graphs. If either of these attempts succeeds then an overall decoding success is achieved. Only if both attempts fail does the overall decoding fail.

Fig. 4.3 depicts the BLERs of the same sequence of constructed codes used in Fig. 4.2, but with combined decoding. As can be seen, performing the combined decoding on both graphs can result in significant gain over the individual graphs as well as the superior one. This is because the two decoders complement each other, with one correcting the errors in cases where the other has not been able to, thereby resulting in a superior combined decoding BLER.

It is possible to partially mitigate the additional decoding complexity of the combined decoding method due to decoding on both graphs rather than just one. To this end, note that for any rate of $C_{n}$, the receiver knows which of $\mathbf{G}_{n}$ or $\mathbf{H}_{n}$ has superior performance. So it can decode on the one with superior performance first, avoiding an unnecessary decoding attempt on the other graph if the decoding on the superior graph succeeds. We can also see that at very high rates, combined decoding gives negligible gain over decoding on $\mathbf{H}_{n}$, and at very low rates decoding only on $\mathbf{G}_{n}$ is sufficient. For example, from Fig. 4.2, at an SNR of $3 \mathrm{~dB}$, if rate of $C_{n}$ is greater than 0.89 we only need to decode on $\mathbf{H}_{n}$, if rate of $C_{n}$ is less than 0.76 we only need to decode on $\mathbf{G}_{n}$, and otherwise we should decode on both. In practice, to further limit decoding complexity, it is better to pick up an intermediate rate, $R_{i}$, and only perform combined decoding when rate of $C_{n}$ is greater than $R_{i}$. By selecting $R_{i}$ to be large there will be a degradation in performance while small values of $R_{i}$ will result in fully benefiting from the combined decoding and thereby improving performance. 


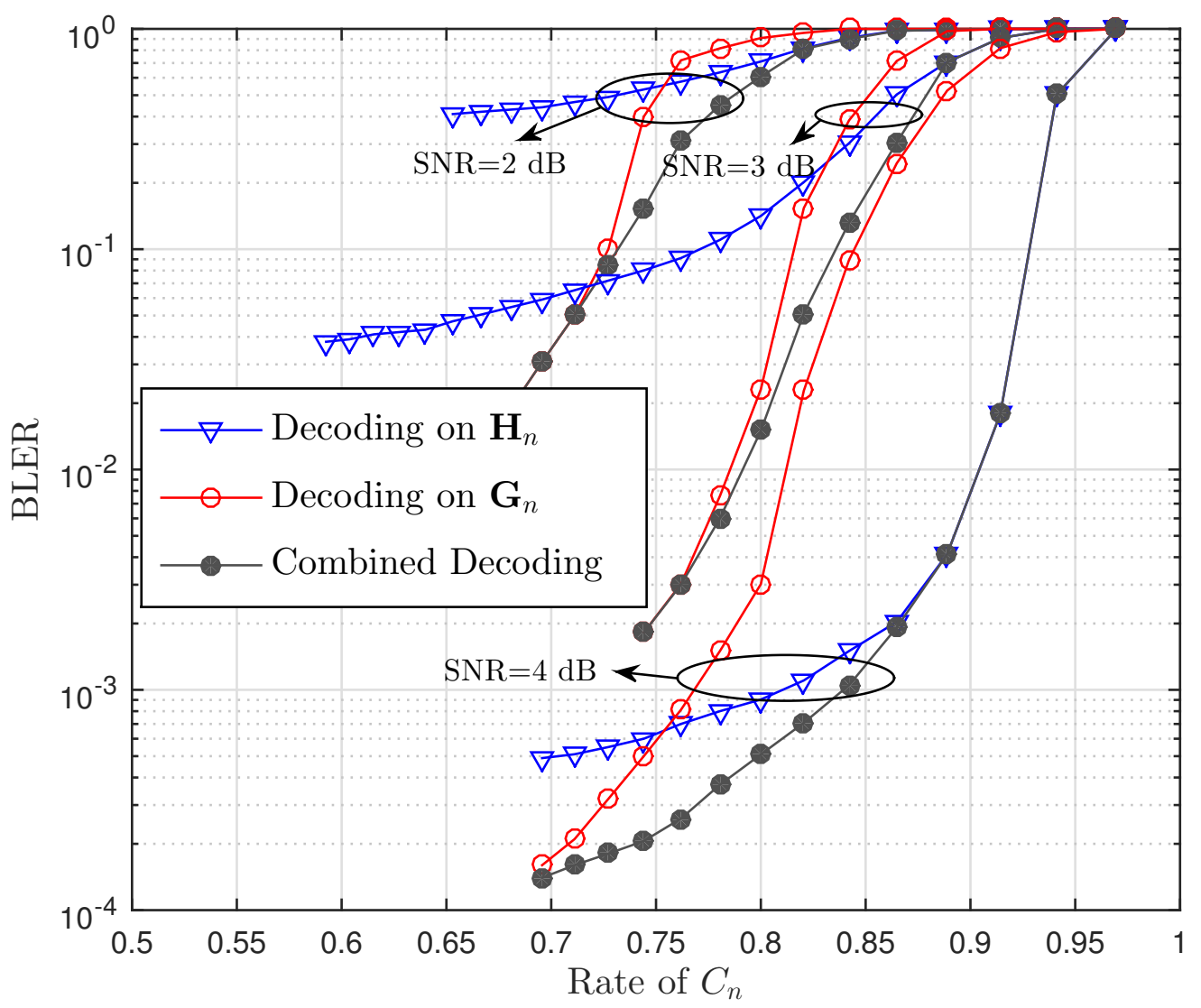

Figure 4.3: BLER of the three decoding methods for the code sequence $\left\{C_{n}\right\}$ in terms of their corresponding rates.

\subsubsection{IR-HARQ scheme}

Once the codes $C_{n}$ and their generator and parity check matrices $\mathbf{G}_{n}$ and $\mathbf{H}_{n}$ have been constructed for a given specific rates $R_{h}$ and $R_{g}$, we employ them in an IR-HARQ scheme as follows. A message word of length $K$ is to be transmitted to the receiver. This message word is encoded with the code $C_{K}$ and the code word is transmitted over the channel. At the receiver side, the decoding is attempted. If decoding fails, additional code bits corresponding the codes $C_{n}, n=K+1, \ldots$ are transmitted, one at a time, with decoding attempted after each bit, until decoding is successful ${ }^{1}$. To investigate the effect of combined decoding on the performance of the system, we choose an intermediate rate, $R_{i}, R_{g} \leq R_{i} \leq$

\footnotetext{
${ }^{1} \mathrm{~A}$ more practical method is to send additional code bits in small clusters, with decoding attempted after each cluster. This increased granularity will result in a degradation in throughput performance which will be negligible if the cluster size is sufficiently small relative to $K$
} 
$R_{h}$ and determine $n_{i}$ as $n_{i}=K / R_{i}$. For the code lengths $n>n_{i}, C_{n}$ is only decoded on $\mathbf{G}_{n}$. For $n<n_{i}$ the combined decoding on both $\mathbf{G}_{n}$ and $\mathbf{H}_{n}$ is used. The IR-HARQ scheme is then described by the three rates $R_{h}, R_{i}, R_{g}$ and is denoted by IR-HARQ $\left(R_{h}, R_{i}, R_{g}\right)$.

\subsection{Simulation Results}

In this section we present the simulation results for the proposed IR-HARQ scheme. The underlying channel is BI-AWGN with binary $\{ \pm 1\}$ phase shift keying (PSK). SNR is defined as $10 \log _{10} \frac{1}{N_{0}}$, where $N_{0}$ is one-sided power spectral density of the AWGN channel. The throughput is defined as $T=\frac{K}{\bar{N}}$ where $\bar{N}$ is the average number of code bits required for successful decoding. We have designed the degree distribution of the LDPC code of rate $R_{h}$ by Gaussian approximation (GA) [51], trying to maximize the decoding threshold. The degree distribution of the LDGM code of rate $R_{g}$ is also designed by the Gaussian approximation method similar to that of [52]. The message word length is considered to be $K=4096$.

We begin with an investigation into the effects of the rate of LDPC mother code, $R_{h}$, on the throughput performance of the IR-HARQ system. Fig. 4.4 shows the throughput results of the IR-HARQ $\left(R_{h}, R_{i}, 0.5\right)$ for different values of $R_{h}$, with $R_{i}$ chosen properly according to the performances of the three decoding methods. The IR-HARQ scheme with $R_{h}=1$ is equivalent to a rate-1 LDPC code which is as if there was no LDPC code actually being used. In this case, the decoder always decodes on the generator graph, so $R_{i}=1$, and the throughput degrades at high SNRs due to the overhead issue of LDGM codes. As the rate of the LDPC mother code decreases, there are two different factors that affect the throughput. Firstly, if the rate of the LDPC code is too small, the throughput performance degrades at high SNRs due to the performance degradation of the highly-punctured LDPC codes. Secondly, the lower the rate of the LDPC code, the more degradation it causes to the generator matrix of the extended LDGM code. To see this, note that according to (4.9) only the first $K$ columns of $\mathbf{G}_{N_{h}}$ can be generated according to the desired degree distribution of the LDGM code, $\lambda^{g}(x)$, while the next $N_{h}-K$ columns of the generator matrix are deterministic and can not follow a desired degree distribution. These columns appear in all generator matrices $\mathbf{G}_{n}, n \geq N_{h}$. As the rate $R_{h}$ decreases the number of these columns increases, and therefore the throughput drops off at lower SNRs. For example for $K=4096$ and $R_{h}=0.6$ there are 2730 of these columns imposed to the generator matrices, $\mathbf{G}_{n}$. However, at a rate of $R_{h}=0.93$ it seems that the LDPC code can be punctured to higher 


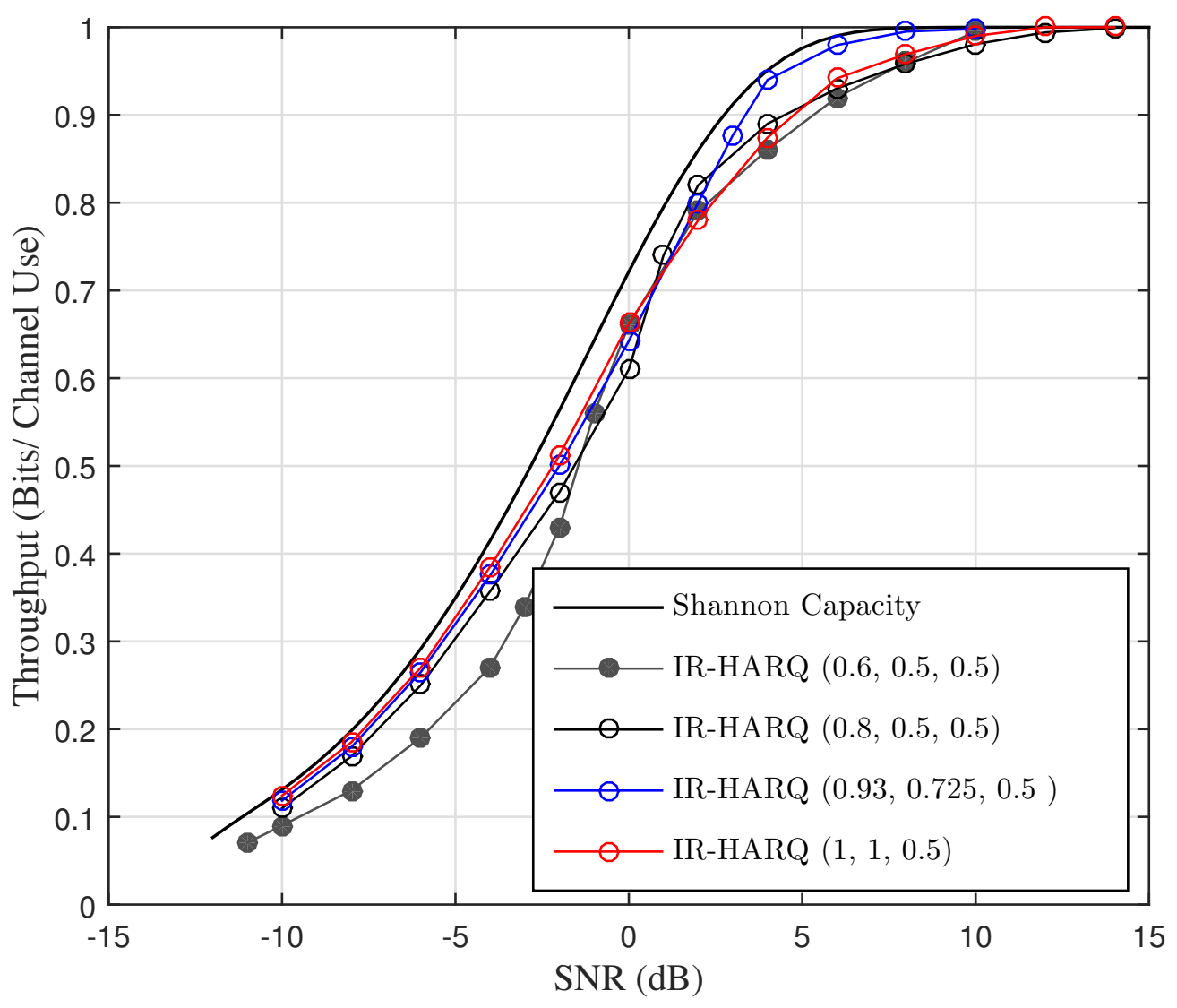

Figure 4.4: The effect of the rate, $R_{h}$, of the LDPC mother code on the throughput performance of an IR-HARQ $\left(R_{h}, R_{i}, R_{g}\right)$ system.

rates while having only a small effect on the degree distribution of the generator matrices by only imposing 308 columns. Therefore it is advisory to choose a fairly high $R_{h}$ for the $\operatorname{IR-HARQ}\left(R_{h}, R_{i}, R_{g}\right)$.

It is also useful to see what kind of effects the rate, $R_{g}$, for which the degree distribution of the LDGM code is optimized, will have on the throughput performance. Fig. 4.5 shows an IR-HARQ $\left(0.93, R_{i}, R_{g}\right)$ for different values of $R_{g}$, with $R_{i}$ chosen as mentioned. As can be seen in Fig. 4.5, the performance degrades at intermediate SNRs as $R_{g}$ decreases. This is because codes designed for low rates tend to not perform well at intermediate to high rates. Fig. 4.6 shows the effect of the rate $R_{i}$ on the throughput performance. The throughput results for an IR-HARQ $\left(0.93, R_{i}, 0.5\right)$ is shown for different choices of $R_{i}$. Remember that 


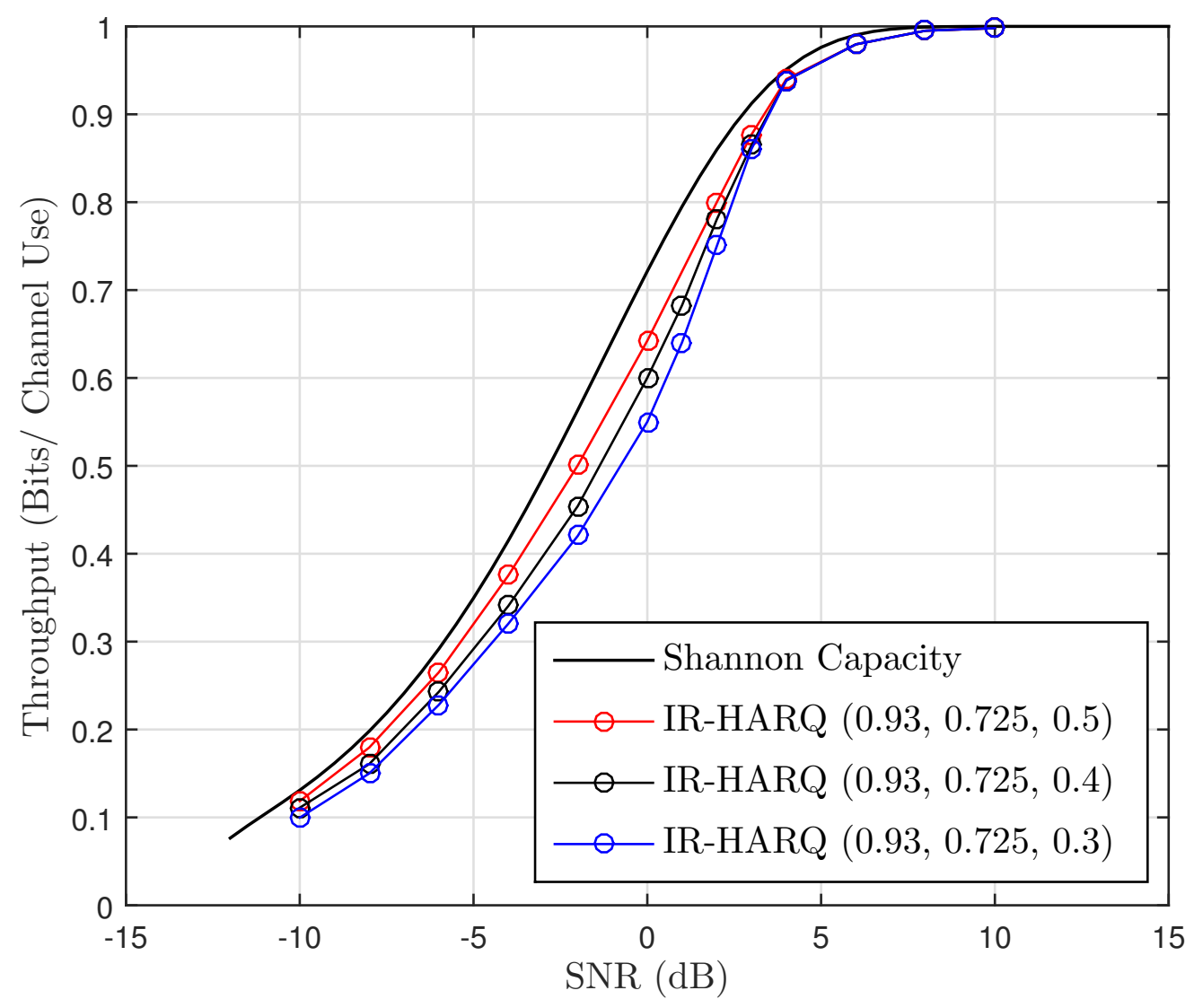

Figure 4.5: The effect of the rate, $R_{g}$, of the LDGM code on the the throughput performance of an IR-HARQ $\left(R_{h}, R_{i}, R_{g}\right)$ system.

for the rates of $C_{n}$ larger than $R_{i}$ the combined decoding is used at the receiver. A choice of $R_{i}=0.93$ is equivalent to decoding only on $\mathbf{G}_{n}$ immediately after the decoding of the LDPC mother code has failed. As it can be seen by decreasing $R_{i}$ improvements on the throughput can be observed due to the combined decoding. At a rate of $R_{i}=0.725$ the gain over $R_{i}=0.93$ is almost $1.5 \mathrm{~dB}$ at a throughput of 0.94 . Decreasing $R_{i}$ more does not result in additional throughput improvements. It also increases the decoding complexity because the combined decoding is used more often.

We compare the proposed IR-HARQ scheme, with appropriately chosen rates $\left(R_{h}, R_{i}, R_{g}\right)$, with related IR-HARQ works in Fig. 4.7. The proposed IR-HARQ scheme uses the following optimized degree distributions for the LDPC and LDGM codes chosen for rates 


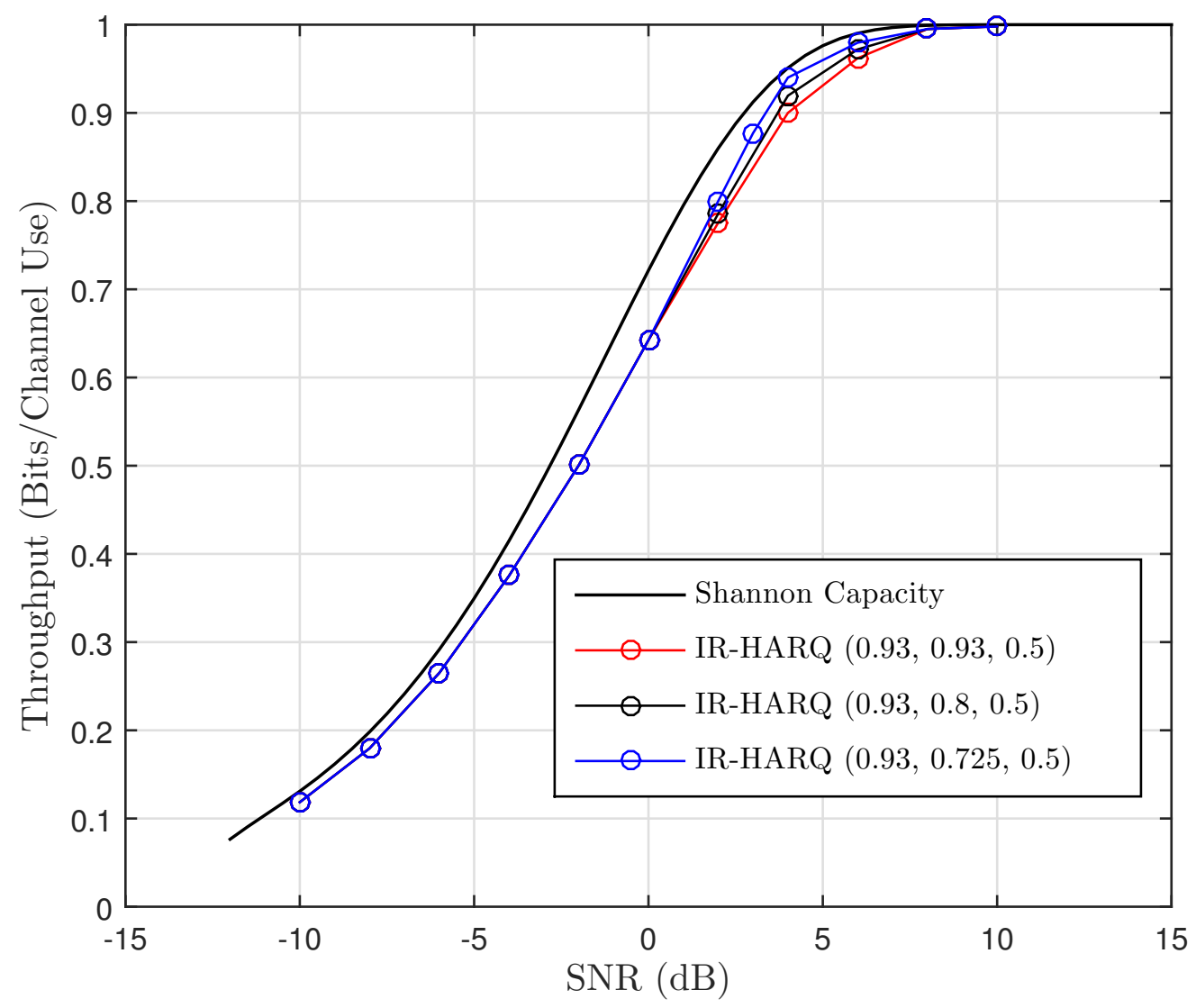

Figure 4.6: The effect of the rate $R_{i}$ on the the throughput performance of an IR$\operatorname{HARQ}\left(R_{h}, R_{i}, R_{g}\right)$ system.

$R_{h}=0.93$ and $R_{g}=0.5$ :

$$
\begin{aligned}
\lambda^{h}(x) & =0.196 x^{2}+0.382 x^{3}+0.141 x^{4}+0.029 x^{5}+0.013 x^{6} \\
& +0.076 x^{7}+0.008 x^{8}+0.026 x^{9}+0.016 x^{10}+0.003 x^{11} \\
& +0.022 x^{12}+0.009 x^{13}+0.016 x^{15}+0.018 x^{16} \\
& +0.025 x^{18}+0.002 x^{19}+0.018 x^{20}
\end{aligned}
$$




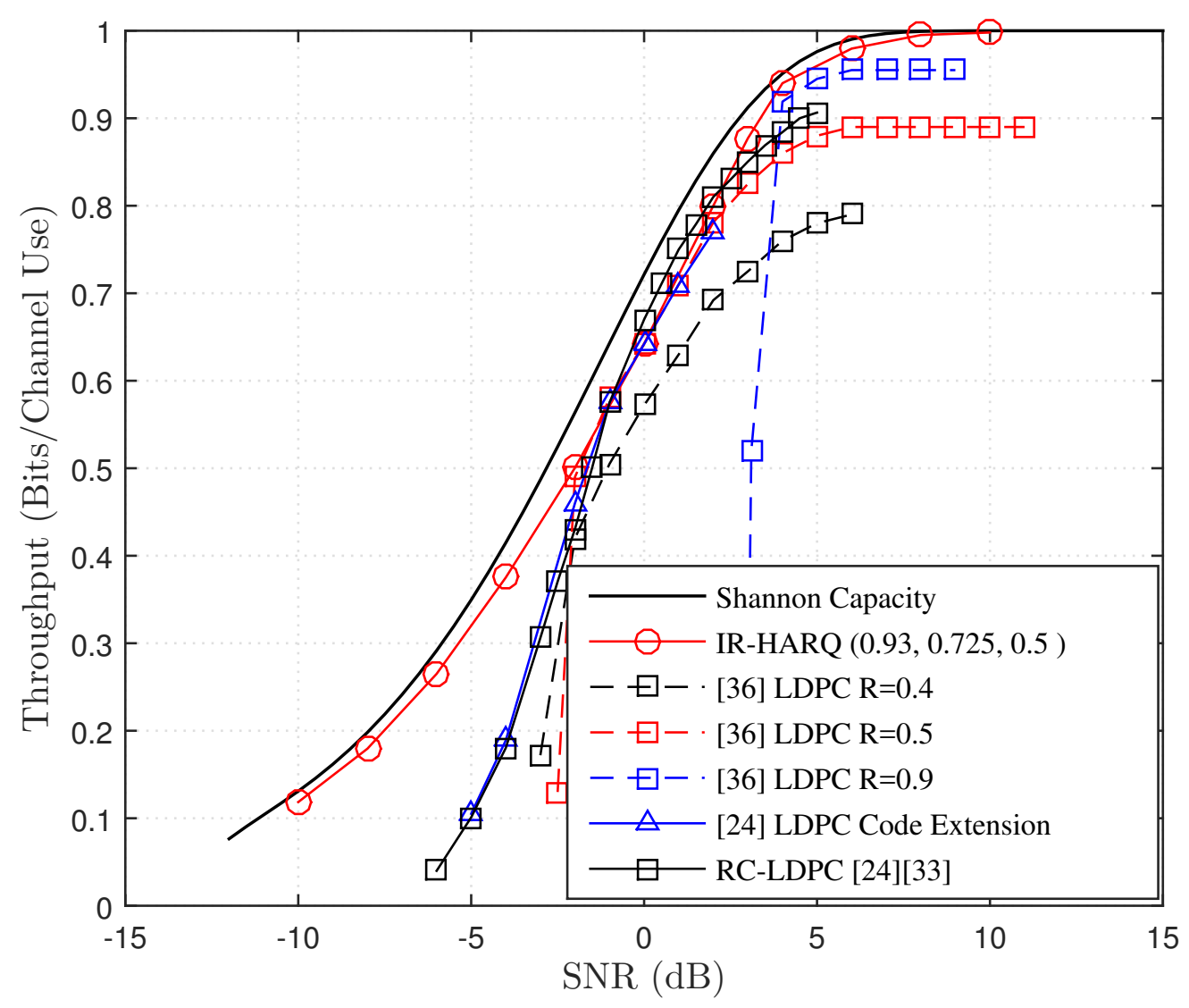

Figure 4.7: Throughput result of different IR-HARQ schemes.

and

$$
\begin{aligned}
\lambda^{g}(x) & =0.0059 x+0.4877 x^{2}+0.0336 x^{3}+0.2382 x^{4} \\
& +0.0059 x^{5}+0.0942 x^{8}+0.0486 x^{14} \\
& +0.0178 x^{30}+0.0353 x^{33}+0.0328 x^{200}
\end{aligned}
$$

The scheme in [36] which uses random puncturing suffers from a throughput drop-off issue at lower SNRs making the scheme hard for universal capacity approaching IR-HARQ applications. The performance of the scheme proposed in [24], which uses puncturing and extending of an LDPC mother code, is also shown. This schemes works well at intermediate SNRs but is less attractive at high and low SNRs. We also modified the scheme 


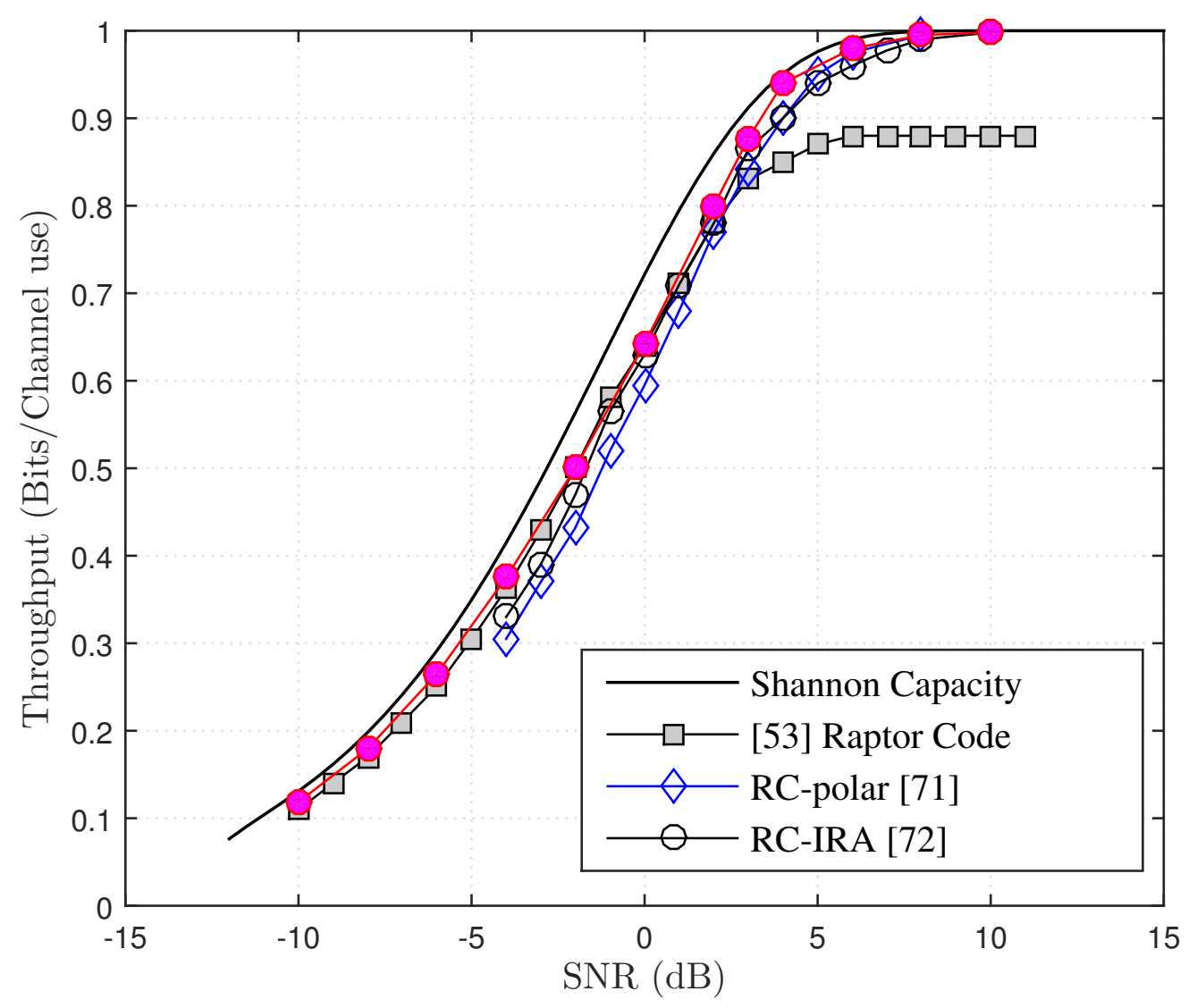

Figure 4.8: Throughput result of different IR-HARQ schemes.

proposed in [24] to use Ha's puncturing algorithm for finite length LDPC codes with an optimized degree distribution. The result, labeled as RC-LDPC [24] [33], outperforms the result in [24] at intermediate SNRs, and even slightly outperforms our scheme. However, the performance is still poor at low and high SNRs.

Fig. 4.8 compares the performance of the proposed IR-HARQ scheme to a scheme based on Raptor codes. The Raptor code corresponds to a concatenation of a high rate LDPC code and the LT code [53]. As can be seen, the Raptor code performs quite well at lower SNRs while leaning away from the capacity at higher rates. We have also compared the throughput performance of the proposed method with that of RC-polar codes [71], as well as RC irregular repeat accumulate (RC-IRA) codes [72]. In case of the RC-polar codes, the low complexity successive cancellation decoder is used, which is an advantage of that 
scheme over the proposed work but the performance is inferior. As can be seen, the proposed IR-HARQ scheme can get as close $0.3 \mathrm{~dB}$ to the capacity, while not getting more than $1 \mathrm{~dB}$ away from it for any SNRs.

\subsection{Conclusion}

A new framework for designing IR-HARQ schemes is proposed by designing a sequence of RC codes $\left\{C_{n}\right\}$ whose nature changes from LDPC to LDGM codes as their rate decreases. Therefore, the proposed RC codes, referred to as RC-LDPC/LDGM codes, can be used for IR-HARQ schemes that can benefit from the advantages of both IR-HARQ schemes based on LDPC and LDGM codes. It is shown that the combined decoding of the codes on both their generator and parity check graphs can result in significant coding gain compared to decodings on individual graphs. The proposed IR-HARQ shows promising capacityapproaching performance for a very wide range of SNRs. This comes at the expense of an increased decoding complexity and a small performance loss at the intermediate SNRs. 


\section{Chapter 5}

\section{Polar Codes for IR-HARQ}

\subsection{Introduction}

Recently proposed by Arikan [54], polar codes are the first class of structured channel codes which can provably achieve the capacity of binary input memoryless output symmetric (BIMOS) channels under successive cancellation (SC) decoding. Although the capacity achieving property of polar codes is an asymptotic attribute, their performance and code design for finite lengths have been studied as well (e.g. [57], [73]). Motivated by the theoretical fixed-rate capacity-achieving property, it is natural to apply polar codes to IR-HARQ. Puncturing schemes to produce RC polar codes are proposed in [67], [68]. The authors in [71] used these methods and designed a IR-HARQ scheme based on polar codes. Their proposed scheme uses puncturing and a selective repetition of the message bits. The reported results suggest that despite the non-promising fixed rate performance of finitelength polar codes, the throughput performance can remain close to the Shannon capacity, when used with IR-HARQ.

In this chapter we focus on designing polar codes for IR-HARQ schemes. Our proposed scheme uses both puncturing and extending. We propose a novel puncturing algorithm and we develop an algorithm to extend the polar codes in such a way that its goal is to maximize the throughput of the system. We extend the polar code by selecting code bits chosen from a finite set of linear combinations of message bits; each combination corresponds to each node in the PC graph (to be defined shortly), while [71] allows only a subset of this set, i.e. the message bits. We also show how the proposed extending can be applied to a polar code which is punctured to an arbitrary rate.

The rest of the chapter is organized as follows. In Section 5.2 we present preliminaries of polar codes, their encoding and decoding, and code design. We present our heuristic 
algorithm for puncturing polar codes in Section 5.3. In Section 5.4 we present an algorithm for extending polar codes for IR-HARQ schemes. Section 5.5 presents the simulation results for different types of puncturing and extending methods. It also compares the best IR-HARQ code with the previous IR-HARQ schemes based on polar codes. Conclusions and topics for future research are provided in Section 5.6.

\subsection{Polar codes}

A polar code with length $N=2^{n}$ is a linear block code whose generator matrix is $\mathbf{G}_{N}=\mathbf{F}^{\otimes n}$, where $\mathbf{F}=\left[\begin{array}{ll}1 & 0 \\ 1 & 1\end{array}\right]$ and $\otimes$ denotes the Kronecker product. ${ }^{1}$ For example, when $N=8$,

$$
\mathbf{G}_{8}=\left[\begin{array}{llllllll}
1 & 0 & 0 & 0 & 0 & 0 & 0 & 0 \\
1 & 1 & 0 & 0 & 0 & 0 & 0 & 0 \\
1 & 0 & 1 & 0 & 0 & 0 & 0 & 0 \\
1 & 1 & 1 & 1 & 0 & 0 & 0 & 0 \\
1 & 0 & 0 & 0 & 1 & 0 & 0 & 0 \\
1 & 1 & 0 & 0 & 1 & 1 & 0 & 0 \\
1 & 0 & 1 & 0 & 1 & 0 & 1 & 0 \\
1 & 1 & 1 & 1 & 1 & 1 & 1 & 1
\end{array}\right] .
$$

Let $\mathcal{A}$ be a set of cardinality $K$ containing a subset of the row indices of $\mathbf{G}_{N}$. We refer to this set as the information set. The complementary set $\mathcal{A}^{c}$ is referred to as the frozen set. Let $\mathbf{u}=\left[u_{1}, \ldots, u_{N}\right]$ be a binary vector of size $1 \times N$. The $K$ information bits are placed in those elements of $\mathbf{u}$ corresponding to the set $\mathcal{A}$, and deterministic values (typically zeros) are placed in the other $N-K$ elements. The codeword corresponding to an information

\footnotetext{
${ }^{1}$ In [54] the generator matrix of the polar code is defined as $\mathbf{G}_{N}=\mathbf{B}_{N} \mathbf{F}^{\otimes n}$, where $\mathbf{B}_{N}$ is a permutation matrix known as the bit reversal permutation matrix. However $\mathbf{B}_{N}$ is just a permutation matrix, and thus, for simplicity we can assume that the message vector is already permutated and the generator matrix can be considered as $\mathbf{G}_{N}=\mathbf{F}^{\otimes n}$. This representation of the generator matrix is also useful in developing the extending algorithms using the $\mathrm{PC}$ graph.
} 
vector $u_{\mathcal{A}}$ is then calculated as

$$
\mathbf{c}=\mathbf{u G}_{N}
$$

We consider a BIMOS channel $\mathcal{W}$ with binary input alphabet, output alphabet $\mathcal{Y}$ and the transition probabilities $f_{W}(y \mid 0)$ and $f_{W}(y \mid 1)$ for $y \in \mathcal{Y}$. The codeword $\mathbf{c}$ is transmitted over $\mathcal{W}$ and the channel output vector $\mathbf{y}=\left[y_{1}, \ldots, y_{N}\right]$ is received. Note that the term "polar code" refers to a specific instance of the set $\mathcal{A}$, chosen according to the polar rule which chooses the indices of the bit-channels (suitably defined in [54]) with the largest capacities (for more details see [54]). A polar code is completely described by $(N, K, \mathcal{A})$. After describing the encoding and decoding algorithms for polar codes in Section 5.2.1, a method for determining $\mathcal{A}$ is presented in Section 5.2.2.

\subsubsection{Encoding and Decoding}

Encoding and decoding of polar codes is most usefully described in terms of a graphical representation of the generator matrix, using what we refer to as the polar code (PC) graph. Fig. 5.1 depicts the PC graph for the polar code of length $N=8$, corresponding to $\mathbf{G}_{8}$ given in (5.1). We represent each node in the PC graph of polar code of length $N=2^{n}$ by $(i, j)$, where $i \in\{1, \ldots, N\}$ and $j \in\{0, \ldots, n\}$ are the row and column indices of the node in the graph. The rows are numbered from top to bottom, and the columns are numbered from left to right. Numbering of the columns starts with zero rather than one for simplicity in describing the extending algorithm in Section 5.4. Starting with labeling the nodes in column 0 by the message bits $u_{1}, \ldots, u_{N}$, and the nodes in column $n$ by the code bits $c_{1}, \ldots, c_{N}$, each node in the graph corresponds to a linear combination of the message bits. Let $v_{i, j}$ denote the value of this combination at node $(i, j)$, with $v_{i, 0}=u_{i}$ and $v_{i, n}=c_{i}$. Encoding is carried out on the PC graph by applying the message bits to the nodes in the leftmost column, so $v_{i, 0}=u_{i}$. These bits are then sent to the next column. Calculation of the bit values are calculated according to the butterfly shapes in the graph. In each butterfly the right-hand top node is equal to the summation of the bits in the two nodes on the left-hand side, and the right-hand bottom node is equal to the left-hand bottom node. The bit values are propagated to the right until the code bits $c_{i}=v_{i, n}$ are calculated. Note that during the encoding process no values are passed along the edges that slope down to the right on the PC graph (indicated by dashed lines in Fig. 5.1). 


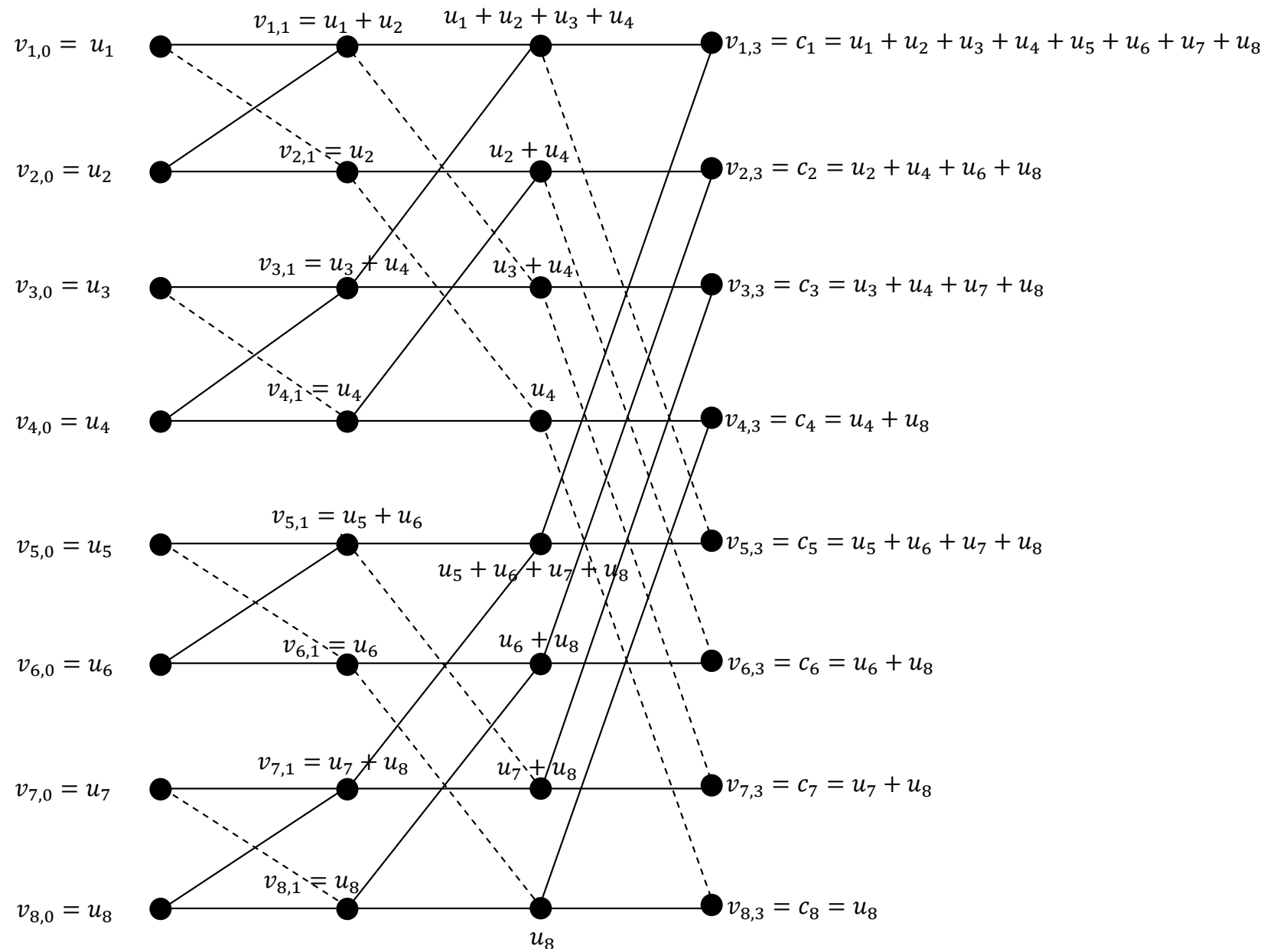

Figure 5.1: The PC graph of a polar code with length $N=8$. 
Arikan proposed a SC decoding algorithm for polar codes [54] that can be described by the PC graph. The channel log-likelihood ratios (LLRs), $\lambda\left(y_{i}\right)=\ln \frac{f_{W}\left(y_{i} \mid 0\right)}{f_{W}\left(y_{i} \mid 1\right)}$ for code bit $c_{i}, i=1, \ldots, N$, are calculated and applied to the rightmost column, so $\lambda_{i, n}=\lambda\left(y_{i}\right)$. These LLRs propagate leftwards through the graph. We say that a node $(i, j), j<n$, is of type I if it is in the upper-left corner of a butterfly, and type II if it is in the lower-left corner. Thus

$$
\operatorname{type}(i, j)= \begin{cases}\mathrm{I} & \text { if }\left\lfloor\frac{i-1}{2^{j}}\right\rfloor \equiv 0 \bmod 2 \\ \text { II } & \text { if }\left\lfloor\frac{i-1}{2^{j}}\right\rfloor \equiv 1 \bmod 2\end{cases}
$$

where $\lfloor x\rfloor$ is the largest integer not greater than $x$. The LLR of the type-I node $(i, j)$ is calculated according to

$$
\begin{aligned}
\lambda_{i, j} & =\ln \frac{e^{\lambda_{i, j+1}} e^{\lambda_{i^{\prime}, j+1}}+1}{e^{\lambda_{i, j+1}}+e^{\lambda_{i^{\prime}, j+1}}} \\
& =2 \tanh ^{-1}\left(\tanh \left(\frac{\lambda_{i, j+1}}{2}\right) \tanh \left(\frac{\lambda_{i^{\prime}, j+1}}{2}\right)\right)
\end{aligned}
$$

where $i^{\prime}$ is the row at the lower edge of the butterfly containing $(i, j)$ and $(i, j+1)$ as the upper edge, and the LLR of the type-II node $\left(i^{\prime}, j\right)$ is given by

$$
\lambda_{i^{\prime}, j}=\lambda_{i^{\prime}, j+1}+\left(1-2 \hat{v}_{i, j}\right) \lambda_{i, j+1}
$$

where $\hat{v}_{i, j}$ is a hard estimate of $v_{i, j}$. To calculate $\hat{v}_{i, j}$ it is necessary to process nodes in a specific order when decoding. In particular, LLRs are only passed upward to the left at first, until $\lambda_{1,0}$ is calculated, and a hard decision for $\hat{v}_{1,0}=\hat{u}_{1}$ is made using $\lambda_{1,0}\left(\hat{v}_{1,0}\right.$ is 0 if $\lambda_{1,0}>0$ and 1 otherwise). Only then can $\lambda_{2,0}$ be calculated by using (5.5) along with $\hat{v}_{1,0}$. Then $\hat{v}_{1,0}$ and $\hat{v}_{2,0}$ can be passed back through the graph so that $\hat{v}_{1,1}$ and $\hat{v}_{2,1}$ can be determined using the same procedure as the encoder. With $\hat{v}_{1,1}$ and $\hat{v}_{2,1}$ known, the decoder can use (5.5) to calculate $\lambda_{3,1}$ and $\lambda_{4,1}$, allowing $\hat{v}_{3,0}$ and $\hat{v}_{4,0}$ to be eventually calculated. By continuing this procedure the message bits can be successfully decoded in the order of $\hat{u}_{1}, \hat{u}_{2}, \ldots$, and, under the assumption that correct decisions are made, their effects can be cancelled, enabling reliable estimation of as-of-yet undecoded message bits. Note that since the values of the frozen bits, $u_{i} i \in \mathcal{A}^{c}$, are deterministic and known to the receiver, the decoder simply chooses $\hat{v}_{i, 0}=\hat{u}_{i}=u_{i}$ for $i \in \mathcal{A}^{c}$. 


\subsubsection{Code Design}

To design the $(N, K, \mathcal{A})$ polar code it is necessary to find the set of indices for the information bits, $\mathcal{A}$. Although the construction is explicit, it is only efficient for the special case of BEC. There have been research on efficient construction of polar codes for arbitrary channels. The authors in [73]- [74] proposed a density evolution method to approximate the bit-channels from which the information set can be determined in linear time. An efficient algorithm to approximate the bit-channels by having other channels with manageable alphabet size is proposed in [75]. There are other methods to approximate the bit-channels based on Gaussian approximation and min-sum density [76]- [77]. For a comparative study on different polar code construction methods see [78]. In this thesis we use the method in [73], which is based on density evolution, assuming that the all-zero codeword is transmitted. Every node $(i, j)$ in the graph is assigned a probability density denoted by $f_{i, j}(\lambda)$ which is the density of $\lambda_{i, j}$, the LLR calculated at that node. All nodes in the rightmost column are assigned the channel LLR density $f_{W}^{(0)}(\lambda)$, i.e. $f_{i, n}(\lambda)=f_{W}^{(0)}(\lambda)$ for $i=1, \ldots, N$. The density $f_{W}^{(0)}(\lambda)$ is defined as follows. For a channel with transition probabilities $f_{W}(y \mid 0)$ and $f_{W}(y \mid 1)$, define $\lambda(y)=\ln \frac{f_{W}(y \mid 0)}{f_{W}(y \mid 1)}$. The channel LLR density, $f_{W}^{(0)}(\lambda)$, is defined as the probability density function of $\lambda(y)$, when $y$ has the density $f_{W}(y \mid 0)$. For a binary input AWGN channel with antipodal signaling $\{ \pm 1\}$ and noise variance $\sigma^{2}$, we have $\lambda(y)=2 y / \sigma^{2}$ and

$$
f_{W}^{(0)}(\lambda)=\frac{1}{\sqrt{8 \pi / \sigma^{2}}} \exp \left(-\frac{\left(\lambda-2 / \sigma^{2}\right)^{2}}{8 / \sigma^{2}}\right)
$$

The algorithm calculates the densities at all the other nodes, from right to left, according to

$$
f_{i, j}(\lambda)= \begin{cases}f_{i, j+1}(\lambda) \circledast f_{i^{\prime}, j+1}(\lambda) & \text { if type }(i, j)=\mathrm{I} \\ f_{i, j+1}(\lambda) \circledast f_{i^{\prime}, j+1}(\lambda) & \text { if } \operatorname{type}(i, j)=\mathrm{II}\end{cases}
$$

where $(i, j+1)$ and $\left(i^{\prime}, j+1\right)$ are the two right neighbor nodes of $(i, j)$, $\circledast$ denotes the convolution of the two densities, and $⿴ 囗 6)$ denotes the operator for calculation of the density at the output of a check node [73]. In particular, if $X$ and $Y$ are two random variables with densities $f_{X}(x)$ and $f_{Y}(y)$, and $Z=2 \tanh ^{-1}\left(\tanh \frac{X}{2} \tanh \frac{Y}{2}\right)$, then the density of $Z$ is written as $f_{Z}(z)=f_{X}(x)$ 困 $f_{Y}(y)$. Each density calculated in the leftmost column, $f_{i, 0}(\lambda)$ is the density of the LLR of bit $u_{i}, \lambda_{i, 0}$, under the assumption that the all-zero codeword was transmitted 
and that $u_{1}, \ldots, u_{i-1}$ were decoded correctly.

The first-error event probability, which is the probability that $u_{i}$ is the first bit to be incorrectly decoded given that all previously decoded bits are correct, is defined as

$$
\begin{aligned}
P_{e}(i) & :=\operatorname{Pr}\left(\hat{u}_{i} \neq u_{i} \mid \hat{u}_{1}=u_{1}, \ldots, \hat{u}_{i-1}=u_{i-1}\right) \\
& =\int_{-\infty}^{0^{-}} f_{i, 0}(\lambda) d \lambda+\frac{1}{2} \int_{0^{-}}^{0^{+}} f_{i, 0}(\lambda) d \lambda
\end{aligned}
$$

These error probabilities can be sorted in ascending order, and the indices of the $K$ bits with the smallest error probabilities can be used for $\mathcal{A}$.

As an aside, it is worth noting that a rate-compatible polar code can be found by fixing $N$ and varying $K$, with $\mathcal{A}_{K}$ the information set for rate $K / N$, chosen as above. In this case $\mathcal{A}_{K}$ will contain all elements of $\mathcal{A}_{K-1}$ plus one additional index. The transmitter and receiver only need to know the order of the indices as determined above to produce codes with any rate that is an integer multiple of $1 / N$. However, this type of RC polar code is not useful for IR-HARQ schemes, where $K$ is normally fixed and $N$ varied. In this case puncturing and extending can be used to produce RC polar codes.

\subsection{Puncturing algorithms}

By transmitting only a subset of the code bits of a rate $K / N$ mother code, puncturing allows for the creation of higher-rate codes. Decoding of punctured polar codes can be accomplished by applying LLR values of zero to the nodes in the rightmost column of the PC graph that correspond to the punctured code bits. The more difficult challenge is determining which bits to puncture to least degrade the performance of the code.

In [68] the authors proposed a stopping tree puncturing algorithm to determine which code bits to puncture to achieve a desired rate. Code bits which depend on the least number of message bits are punctured first. This algorithm is designed for use with a belief propagation (BP) decoder [66], so may not be applicable to SC decoding. The authors in [67] proposed a quasi-uniform puncturing (QUP) algorithm which punctures the code bits in such a way that it tries to keep the distance between any two adjacent punctured code bits as uniform as possible. QUP is shown to possess good properties reflecting the minimum Hamming distance of the punctured code. Neither of these heuristic schemes are necessarily useful for SC decoding in IR-HARQ systems. 
In the context of IR-HARQ, puncturing is better framed as determining in which order to transmit the code bits, with the idea that more "important" code bits should be transmitted first, to increase the likelihood of early successful decoding. However, there are no precise metrics for ranking the relative importance of the code bits that are computationally feasible, so instead we propose another heuristic algorithm. We note that since the generator matrices of the polar codes are involutory, the code bits and message bits are calculated from each other with the same type of linear combination. For example $c_{1}$ is the summation of all message bits while $u_{1}$ is the summation of all code bits, $c_{2}=u_{2}+u_{4}+u_{6}+u_{8}$ while $u_{2}=c_{2}+c_{4}+c_{6}+c_{8}$, and so on. So it seems that there could be a similarity between the reliability of any message bit $u_{i}$ and the importance of the corresponding code bit with the same index $c_{i}$. We therefore conjecture without proof that, just as more reliable message bits are those with indices with smallest first-error probabilities, more important code bits might be those with the same indices. As a result we propose to transmit the code bits in the same order in which the message bit first-error probabilities increase. So if $u_{i}$ has smaller first-error probability than $u_{j}$ then code bit $c_{i}$ is transmitted before code bit $c_{j}$. Note that the problem of designing an optimal puncturing algorithm for finite length polar codes still remains open. The proposed method, as we see in Section V, is nonetheless effective when combined with the extending algorithm, regardless of whether or not the above conjecture could be proved, or is even relevant.

Implementation of this algorithm is straightforward since, as part of the code design process for constructing polar codes using density evolution [73], the first-error event probability of each message bit has already been calculated according to (5.8). Whereas for code construction we were only interested in selecting the $K$ bits with the lowest probabilities, here we are interested in the order of all $N$ bits, ordered from lowest to highest probability. Let $P_{e}(i)$ be the first-error probability of the message bit $u_{i}$. We make an ordered set $\Omega=$ $\left\{\omega_{1}, \ldots, \omega_{N}\right\}$ from $\{1,2, \ldots, N\}$ in such a way that

$$
P_{e}\left(\omega_{i}\right) \leq P_{e}\left(\omega_{i+1}\right) .
$$

The code bits are then transmitted in the order of $c_{\omega_{1}}, c_{\omega_{2}}, c_{\omega_{3}}, \ldots .{ }^{2}$ As an example, suppose we have a polar code of length $N=8$ and we wish to only transmit four code bits. Our

\footnotetext{
${ }^{2}$ It is important to note that, if we were designing a fixed-rate code, it would be prudent to re-select the information set, $\mathcal{A}$, once the puncturing pattern has been determined, but that is not possible if ratecompatibility is required, so is not done here. The originally selected information set of the mother code remains fixed.
} 
algorithm would transmit code bits $c_{8}, c_{7}, c_{6}$ and $c_{4}$, as their corresponding message bits have the smallest first-error probabilities, whereas QUP would transmit $c_{8}, c_{4}, c_{6}$ and $c_{2}$, and stopping tree puncturing would transmit $c_{1}, c_{2}, c_{3}$ and $c_{5}$.

\subsection{Extending algorithms}

Whereas puncturing is a useful tool for constructing higher-rate codes from a lower-rate mother code, extending allows us to generate lower-rate codes from a higher-rate mother code. For linear block codes this is typically achieved by either retransmitting previously transmitted code bits or by adding additional columns to the generator matrix to allow for the creation of new code bits with different parity check equations. Generally speaking, generation of new parity bits gives a more powerful extended code than relying on retransmissions, but comes at the expense of higher decoding complexity. In particular, adding arbitrary columns to the generator matrix would require adding additional nodes and edges to the PC graph. With an increase to the number of edges connected to a node would come an increase of the complexity of the operations that need to be carried out at the nodes over the relative simplicity of (5.4) and (5.5). Furthermore, the repetitive symmetry of the butterfly shapes in the PC graph would also be lost, leading to higher decoding and scheduling complexity.

In [71] the authors proposed a simple extending scheme for polar codes that involves selective transmission and retransmission of the code bits along with the transmission of the uncoded message bits. This simple scheme does not require any significant decoding complexity since decoding is performed on the unaltered PC graph of the mother polar code. The LLR for a retransmitted code bit is added to the LLRs from previous transmissions of that code bit prior to decoding, and the LLR for a transmitted message bit is added to the corresponding LLR produced by the decoder immediately prior to making a hard decision on that bit. This simple scheme is quite effective at high code rates, but performance suffers with low rate extended codes.

In this section we propose a new extending algorithm for polar codes that, like the method in [71], allows for decoding on the unaltered PC graph, while also providing good codes over a wide range of rates. Motivating this technique is the observation that every node in the PC graph corresponds to a linear combination of the message bits and could therefore be considered as a code bit for a linear block code. We therefore propose to extend the polar codes by transmitting the bit values $\left(v_{i, j}\right)$ associated with an appropriately selected subset of 
all of the nodes in the graph, not just those associated with the message bits or the code bits of the polar code. By sending only these bits (as opposed to arbitrary linear combinations of the message bits), SC decoding is still straightforward. Each node $(i, j)$ in the graph must be modified to accept a channel LLR corresponding to transmission of $v_{i, j}$, and this LLR should be added to the LLR computed according to (5.4) or (5.5) before passing it to the neighbors to the left. If $v_{i, j}$ has not been transmitted then a channel LLR of 0 should be used. With this technique it is possible to produce extended polar codes with nearly any rate from $K / N$ down to $K /\left(K+N \log _{2} N\right)$ without having to resort to retransmitting bits.

The problem of designing a good extended polar code of rate $K / N^{\prime}$ that can still be decoded with the low-complexity SC decoder, then, becomes one of choosing which $N^{\prime}$ out of the $N\left(1+\log _{2} N\right)$ potential "code bits" corresponding to all of the nodes in the graph, to transmit to achieve the best performance. For an IR-HARQ scheme, the problem is better formulated as deciding which order to transmit these code bits in so that the throughput is maximized. We refer to this order as the extending sequence.

\subsubsection{Proposed Algorithm}

Suppose we have a mother code of rate $R_{M}=K / N_{M}, N_{M}=2^{n}$, that has been punctured to rate $R_{I}=K / N_{I} \geq R_{M}$. Equivalently, suppose we have a mother code of length $N_{M}$ and we have transmitted a subset of $N_{I}$ of its code bits. We then wish to transmit additional bits until decoding succeeds. The additional bits may be, but are not necessarily, code bits of the mother code, but must correspond to nodes of the PC graph (from any column). The extending sequence $C=\left\{\left(\omega_{1}, d_{1}\right),\left(\omega_{2}, d_{2}\right),\left(\omega_{3}, d_{3}\right), \ldots\right\}$ defines the order in which the additional bits should be transmitted. That is $v_{\omega_{1}, d_{1}}$ should be transmitted first, followed by $v_{\omega_{2}, d_{2}}$, and so on.

As our goal is to produce codes suitable for incremental redundancy schemes, we want to choose the extending sequence that maximizes the throughput. The throughput, $T$, is defined as $T=\frac{K}{\bar{N}}$, where $K$ is the length of the message word and $\bar{N}$ is the average number of code bit transmissions for successful decoding. If we let $\mathcal{E}_{i}$ denote the event that the decoding fails after $i$ code bits (from any column of the PC graph) have been transmitted, 
then

$$
\begin{aligned}
\bar{N} & =\sum_{m=K}^{\infty} m \operatorname{Pr}\left(\left[\bigcap_{i=K}^{m-1} \mathcal{E}_{i}\right] \cap \mathcal{E}_{m}^{c}\right) \\
& =\sum_{m=K}^{\infty} m\left[\operatorname{Pr}\left(\bigcap_{i=K}^{m-1} \mathcal{E}_{i}\right)-\operatorname{Pr}\left(\bigcap_{i=K}^{m} \mathcal{E}_{i}\right)\right] .
\end{aligned}
$$

To increase throughput, we need to decrease $\bar{N}$. Defining $\alpha_{m}=\operatorname{Pr}\left(\bigcap_{i=K}^{m-1} \mathcal{E}_{i}\right)-\operatorname{Pr}\left(\bigcap_{i=K}^{m} \mathcal{E}_{i}\right)$, we would like to choose the extending nodes so that $\sum_{m=K}^{\infty} m \alpha_{m}$ is minimized. However, for an extending sequence of length $N_{E}$ there are $\left(K+N_{M} \log _{2} N_{M}\right)^{N_{E}}$ possible different ways to choose the extending sequence and this grows exponentially with its length, so this optimization problem is hard to solve. Furthermore, even if we could find the optimized extending sequence that maximized the throughput at one signal-to-noise ratio (SNR), it may not be rate compatible with the optimized sequence at another SNR.

We therefore propose a greedy algorithm for extending the code by one bit at a time. Suppose that we have transmitted exactly $m-1$ code bits corresponding to $m-1$ nodes in the PC graph. We want to know which one node to transmit next to help the throughput the most. Given all previous $m-1$ transmitted bits, all $\alpha_{i} i=K, \ldots, m-1$ are determined. We would like to choose the next extending node in such a way that it minimizes $\bar{N}=\sum_{i=K}^{\infty} i \alpha_{i}$. Since $\alpha_{i}$ are fixed for $i<m$, this is equivalent to minimizing $\sum_{i=m}^{\infty} i \alpha_{i}$ subject to $\sum_{i=m}^{\infty} \alpha_{i}=1-\sum_{i=K}^{m-1} \alpha_{i}$. Observing that

$$
\begin{aligned}
& \sum_{i=m}^{\infty} i \alpha_{i} \geq \sum_{i=m}^{\infty} m \alpha_{i} \\
& =m \sum_{i=m}^{\infty} \alpha_{i}=m\left(1-\sum_{i=K}^{m-1} \alpha_{i}\right)
\end{aligned}
$$

this lower bound can be reached by choosing $\alpha_{i}=0 \forall i>m$, and $\alpha_{m}=1-\sum_{i=K}^{m-1} \alpha_{i}$. Since

$$
1-\sum_{i=K}^{m-1} \alpha_{i}=\operatorname{Pr}\left(\bigcap_{i=K}^{m-1} \mathcal{E}_{i}\right)
$$

and 


$$
\alpha_{m}=\operatorname{Pr}\left(\bigcap_{i=K}^{m-1} \mathcal{E}_{i}\right)-\operatorname{Pr}\left(\bigcap_{i=K}^{m} \mathcal{E}_{i}\right)
$$

this implies the next node should be chosen in such a way that it makes $\operatorname{Pr}\left(\bigcap_{i=K}^{m} \mathcal{E}_{i}\right)$ zero. Since we cannot make this probability zero in practice, we try to minimize it by minimizing $\operatorname{Pr}\left(\bigcap_{i=K}^{m} \mathcal{E}_{i}\right)$.

For a general linear block code there is no relationship between the events $\mathcal{E}_{i}$ and $\mathcal{E}_{i-1}$. In other words, mathematically it is possible that decoding the code of length $i-1$ succeeds and that of length $i$ does not, and vice versa. However, in an IR-HARQ scheme, where the decoder decodes after it has received each code bit, the decoder does not start decoding the code of length $i$, unless it has already attempted decoding the code of length $i-1$ and has failed. So for the decoder of an IR-HARQ scheme, we have $\mathcal{E}_{i} \subset \mathcal{E}_{i-1}$, which implies

$$
\operatorname{Pr}\left(\bigcap_{i=K}^{m} \mathcal{E}_{i}\right)=\operatorname{Pr}\left(\mathcal{E}_{m}\right)
$$

Therefore, minimizing $\operatorname{Pr}\left(\bigcap_{i=K}^{m} \mathcal{E}_{i}\right)$ can be done by minimizing $\operatorname{Pr}\left(\mathcal{E}_{m}\right)$, which is simply the block error rate (BLER) after $m$ code bit transmissions. ${ }^{3}$ So we propose to find the extending node in such a way that it reduces the BLER of the extended polar code as much as possible. The BLER of a polar code can be estimated by $1-\prod_{i}\left(1-P_{e}(i)\right)$, where $P_{e}(i)$ is the first-error probability of bit $u_{i}$, corresponding to the LLR calculated at the node $(i, 0)$, and is given by (5.8). This implies that we should choose the extending node such that it will minimize $1-\prod_{i}\left(1-P_{e}(i)\right)$.

Density evolution is used to evaluate the first-error probabilities, using a technique similar to the approach used for polar code design as described in Section II. Associated with each node $(i, j)$ in the PC graph are two densities: the channel LLR density, $f_{i, j}^{(0)}(\lambda)$, and the calculated LLR density, $f_{i, j}(\lambda)$. The channel LLR densities are

\footnotetext{
${ }^{3}$ It is noteworthy that if we did not have an IR-HARQ decoding scheme, (5.14) would not hold mathematically. But we could still make use of it as $\operatorname{Pr}\left(\bigcap_{i=K}^{m} \mathcal{E}_{i}\right) \leq \operatorname{Pr}\left(\mathcal{E}_{m}\right)$, and minimizing $\operatorname{Pr}\left(\bigcap_{i=K}^{m} \mathcal{E}_{i}\right)$ can be turned into minimizing its upper bound, $\operatorname{Pr}\left(\mathcal{E}_{m}\right)$.
} 


$$
f_{i, j}^{(0)}(\lambda)= \begin{cases}f_{W}^{(0)}(\lambda) & \text { if bit } v_{i, j} \text { has been transmitted } \\ \delta(\lambda) & \text { otherwise }\end{cases}
$$

where $f_{W}^{(0)}(\lambda)$ is given by (5.6). The calculated LLR densities in the rightmost column are initialized with $f_{i, n}(\lambda)=f_{i, n}^{(0)}(\lambda)$, and then the other densities are calculated from right to left using (5.7), except that $f_{i, j}(\lambda)$ is also convolved with $f_{i, j}^{(0)}(\lambda)$. Once $f_{i, 0}(\lambda)$ has been calculated, $P_{e}(i)$ can be calculated according to (5.8), except for frozen message bits, where $P_{e}(i)=0 \forall i \in \mathcal{A}^{c}$.

To find the best extending node we could perform an exhaustive search. Each node in the graph can be considered as candidate for the best node, so we consider each node one at a time. For each candidate node we calculate the LLR densities of the message bits, $f_{i, 0}(\lambda)$, the first-error probabilities, and the metric $1-\prod_{i}\left(1-P_{e}(i)\right)$ which is used to represent the BLER under the hypothesis that the candidate node is transmitted next. The candidate node with the smallest metric is chosen as the next extending node.

Because the complexity of this extending search is quite high, it is necessary to reduce the complexity. To this end, we define the depth- $l$ left-neighborhood graph of node $(i, j)$, denoted by $\mathcal{N}_{i, j}^{\overleftarrow{l}}$, as the subgraph of the PC graph consisting of all nodes and edges that can be reached from $(i, j)$ by walking at most $l$ edges only to the left. A similar definition holds for the depth- $l$ right-neighborhood graph of a node, denoted by $\mathcal{N}_{i, j}^{\vec{l}}$. For example $\mathcal{N}_{1,0}^{0}=\{(1,0)\}, \mathcal{N}_{1,0}^{2}=\{(1,0),(1,1),(2,1),(1,2),(2,2),(3,2),(4,2)\}, \mathcal{N}_{3,3}^{\overleftarrow{0}}=\{(3,3)\}$, and $\mathcal{N}_{3,3}^{\overleftarrow{2}}=\{(3,3),(3,2),(7,2),(1,1),(3,1),(5,1),(7,1)\}$.

It is possible to reduce the complexity of calculating the metrics. Once the densities of the current extending sequence have been determined, to update the densities under the hypothesis that candidate node $(\omega, d)$ is transmitted next only requires updates to the densities in the depth- $d$ left neighborhood graph of $(\omega, d)$. Thus instead of calculating all $N\left(1+\log _{2} N\right)$ densities for each hypothesis, we only need to calculate $2^{d+1}-1$.

Even with this modification, however, the complexity of the exhaustive search remains prohibitively large because of the size of the search space, particularly for large $N$. We therefore propose a suboptimal yet effective search algorithm. The algorithm starts by calculating the first-error probability, $P_{e}(i)$, for each message node $(i, 0)$ based on the current densities, and selects the node with the largest probability. This node, referred to as the target node $(\tilde{i}, 0)$, contributes the most to the BLER of code. To reduce the search space, the 


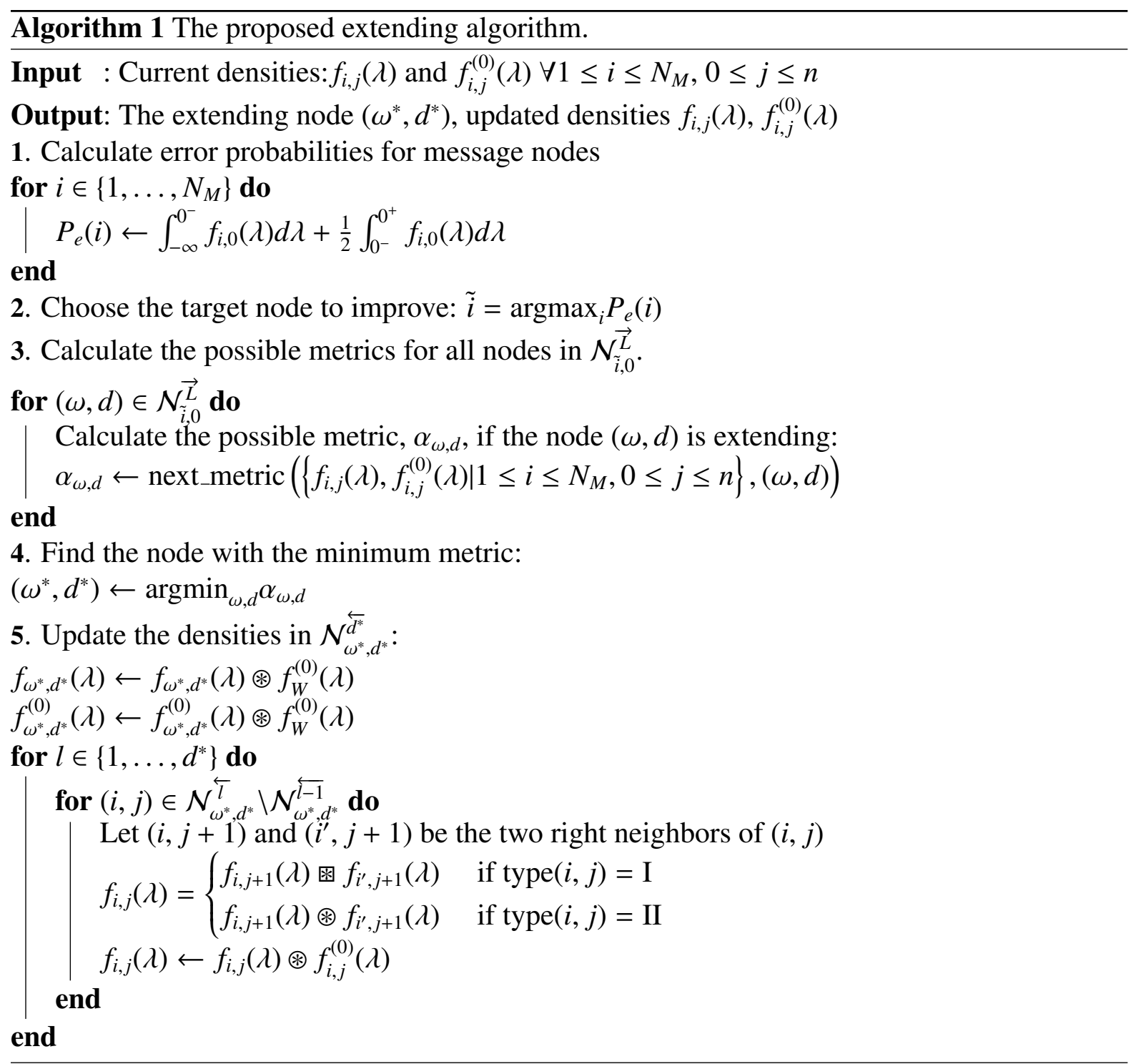

algorithm only searches for the best extending node from within the depth- $L$ right neighborhood graph of the target node (i.e. $\mathcal{N}_{\tilde{i}, 0}^{\vec{L}}$ ), where $L$ is a parameter that limits the scope of the search. Since choosing the extending node from outside of $\mathcal{N}_{\tilde{i}, 0}^{\vec{L}}$ will not have any significant effect on $P_{e}(\tilde{i})$, we can reduce the search space to $2^{L+1}$ nodes instead of $K+N \log _{2} N$.

In summary, the extending sequence is created one node at a time, by searching for the node that would most reduce the BLER of the code. Given an extending sequence of length $m-1$, and the LLR densities $f_{i, j}^{(0)}(\lambda)$ and $f_{i, j}(\lambda)$ corresponding to that sequence, algorithm 1 finds the best node to transmit next, yielding an extending sequence of length $m$ along with the associated updated LLR densities. 


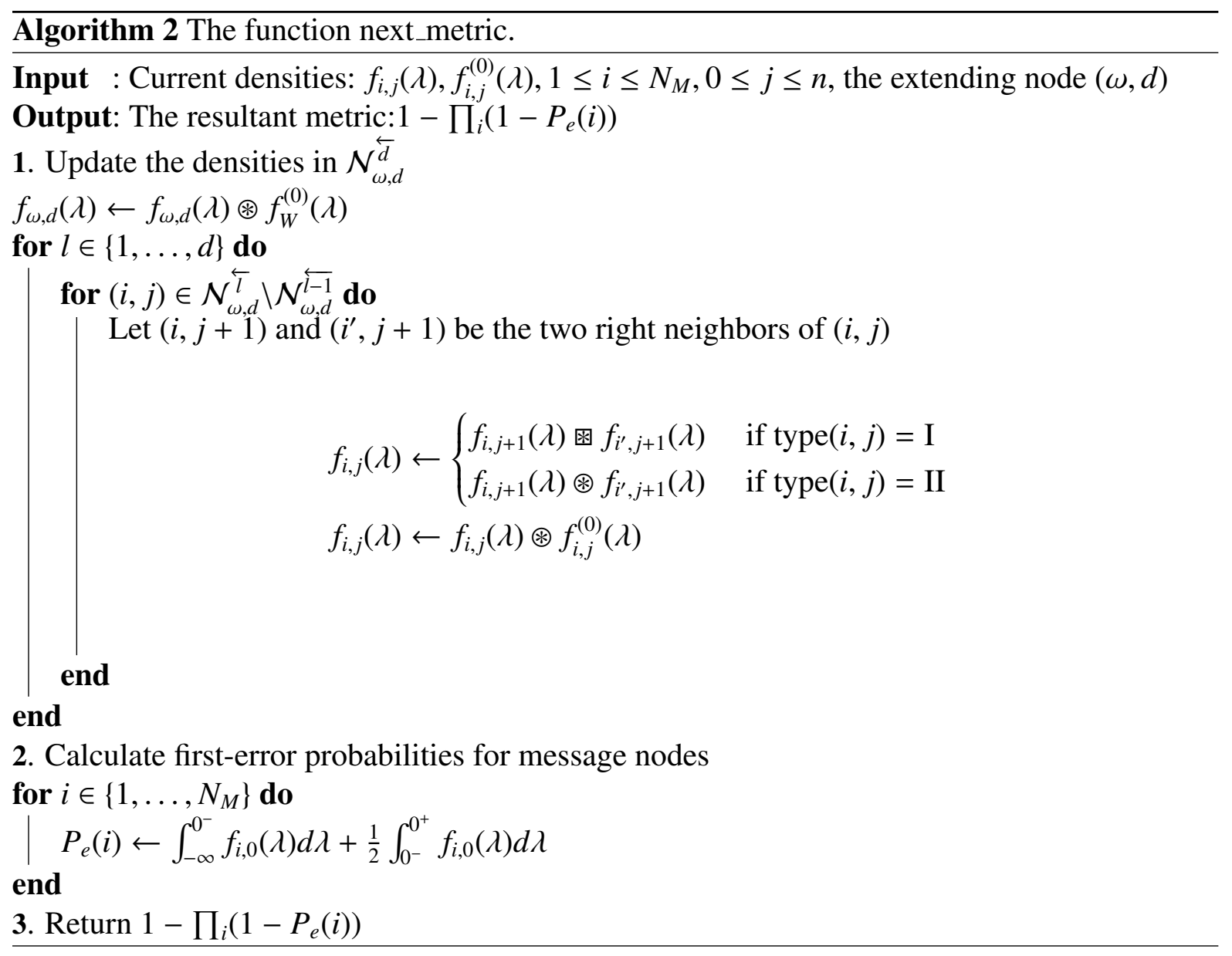

The function next_metric, takes the current densities at the nodes of the graph (corresponding to the as-of-yet constructed extended code) and a node to be considered as the extending node and returns the metric representing the BLER of the extended code for this extending node was used to extend the code. To calculate the resultant metric, it needs to find the updated densities the transmission of the extending node would result in. It updates the densities in the same way as the algorithm does when it chooses the extending node using successive use of (5.7), taking into accounts all the extending nodes in the left neighborhood graph of the chosen node. When the densities are updated, the function returns $1-\prod_{i}\left(1-P_{e}(i)\right)$ as the metric representing the BLER of the extended code.

\subsubsection{A Less Greedy Extending Algorithm}

Algorithm 1 is greedy in the sense that at each step of the algorithm when it makes a decision on the next extending node, it chooses the best one, according to the metric. However, 
this greediness may not result in the best final extending sequence. It may be interesting to see how a less greedy algorithm performs. We propose to introduce a small perturbation in the algorithm: at each step, when it decides on the extending node, it allows a small compromise so as to make it possible to choose nodes other than the best one. To make this work, we choose the extending node randomly from all possible extending nodes with a probability reflecting their possible contribution to improve the next metric. In particular, the next possible metrics for all nodes in the depth- $L$ right neighborhood graph of the target message node are calculated. Then a probability is assigned to each of these nodes such that it is proportional to the inverse of its corresponding next metric. That is, after the algorithm calculates the next metric $\alpha_{\omega, d}$ for each node $(\omega, d)$ in $\mathcal{N}_{\tilde{i}, 0}^{\vec{L}}$, the node is assigned a selecting probability $\rho_{\omega, d}=\frac{\alpha_{\omega, d}^{-1}}{\sum_{j, l} \alpha_{j, l}^{-1}}$. The algorithm chooses the extending node to be $(\omega, d)$ with the probability $\rho_{\omega, d}$. Obviously, the node which Algorithm 1 would choose, i.e., that of the best metric, will have the largest chance of being chosen.

\subsubsection{The proposed IR-HARQ scheme}

In this section we describe how an IR-HARQ scheme can be implemented based on the proposed puncturing and extending algorithms. The proposed IR-HARQ scheme transmits the code bits, each corresponding to one node in the PC graph of the mother polar code. The choice of the code bits to transmit is made in two successive phases. In the first phase, which is the puncturing phase, the code bits are transmitted in the transmission order given by the proposed puncturing pattern in Section III. In the second phase, which is the extending phase, the code bits are transmitted according to the extending sequence given by the extending algorithm in this section. The transition time between the puncturing phase and the extending one is determined by the intermediate rate $R_{I}$.

For a given mother code of rate $R_{M}=K / N_{M}$, a puncturing algorithm is used to determine the transmission order of the $N_{M}$ code bits. Transmission begins with the transmission of the first $K$ code bits (in the specified order), and decoding is attempted at the receiver. If decoding fails, additional code bits are transmitted, one at a time, with decoding attempted after each bit, until decoding is successful. ${ }^{4}$ After $N_{I}$ of the code bits of the polar code have been transmitted, for some $N_{I} \leq N_{M}$, the transmitter begins transmitting bits according to the extending sequence. Let $R_{I}=K / N_{I} \geq R_{M}$ be the intermediate rate after which code bits

\footnotetext{
${ }^{4}$ A more practical method is to send additional code bits in small clusters, with decoding attempted after each cluster. The resulting degradation in throughput arising from this increased granularity will be negligible if the cluster size is sufficiently small relative to $K$.
} 
are selected from the extending sequence instead of according to the puncturing pattern. The transmitter continues to send code bits over the channel until decoding succeeds at the receiver and an acknowledgement is received at the transmitter. ${ }^{5}$ The extending algorithm is run to give a long enough extending sequence to consider the case that the SNR is low and a lot of redundancy code bits may be required for successful decoding. As we will show in the next section, the throughput of the system depends on the chosen mother code rate $R_{M}$, and the intermediate rate $R_{I} \geq R_{M}$, to a significant amount. In the following section we present the simulation results for the proposed IR-HARQ scheme and find a good intermediate rate $R_{I}$ for a given mother code rate $R_{M}$.

\subsection{Simulation Results}

In this section we present Monte-Carlo simulation results to evaluate IR-HARQ schemes which use the different combinations of puncturing and extending methods presented in Sections III and IV. We assume a BIAWGN channel with antipodal signaling $\{ \pm 1\}$. SNR is defined as $10 \log _{10}\left(1 / N_{0}\right)$, where $N_{0}$ is the one-sided power spectral density of the Gaussian noise. Let $K$ be the length of the message word. All mother codes are designed according to $[73]$.

We begin with an investigation into the effects of the puncturing algorithms. Figure 5.2 shows the BLER performance of a polar code of length $N_{M}=2048$ and rate 0.5 that has been punctured to rate 0.7 using each of the three puncturing algorithms described in Section III. While the proposed method outperforms the stopping tree algorithm, the QUP algorithm is even better. In fact, the code produced with QUP is almost as good as a directly generated rate 0.7 Arikan polar code with $N_{M}=2048$ (also shown in Figure 5.2), despite having a shorter codeword length (1462 vs 2048 code bits). Codes produced with QUP also have the advantage over Arikan's codes in that the codeword length is not limited to a power of two. Unfortunately, with QUP the set of frozen bits is determined after the puncturing pattern has been identified. As a result, codes generated with different puncturing lengths are not rate-compatible, and so QUP is not applicable to our system

\footnotetext{
${ }^{5}$ Note that unlike LDPC codes, the SC polar decoder will always produce a valid codeword. In order for it to know if a decoding failure has occurred, one could use an error-detecting code like a CRC code to declare the decoding failure. This would lead to a certain rate loss in the throughput performance, but the rate loss is negligible as the length of the message word increases.
} 


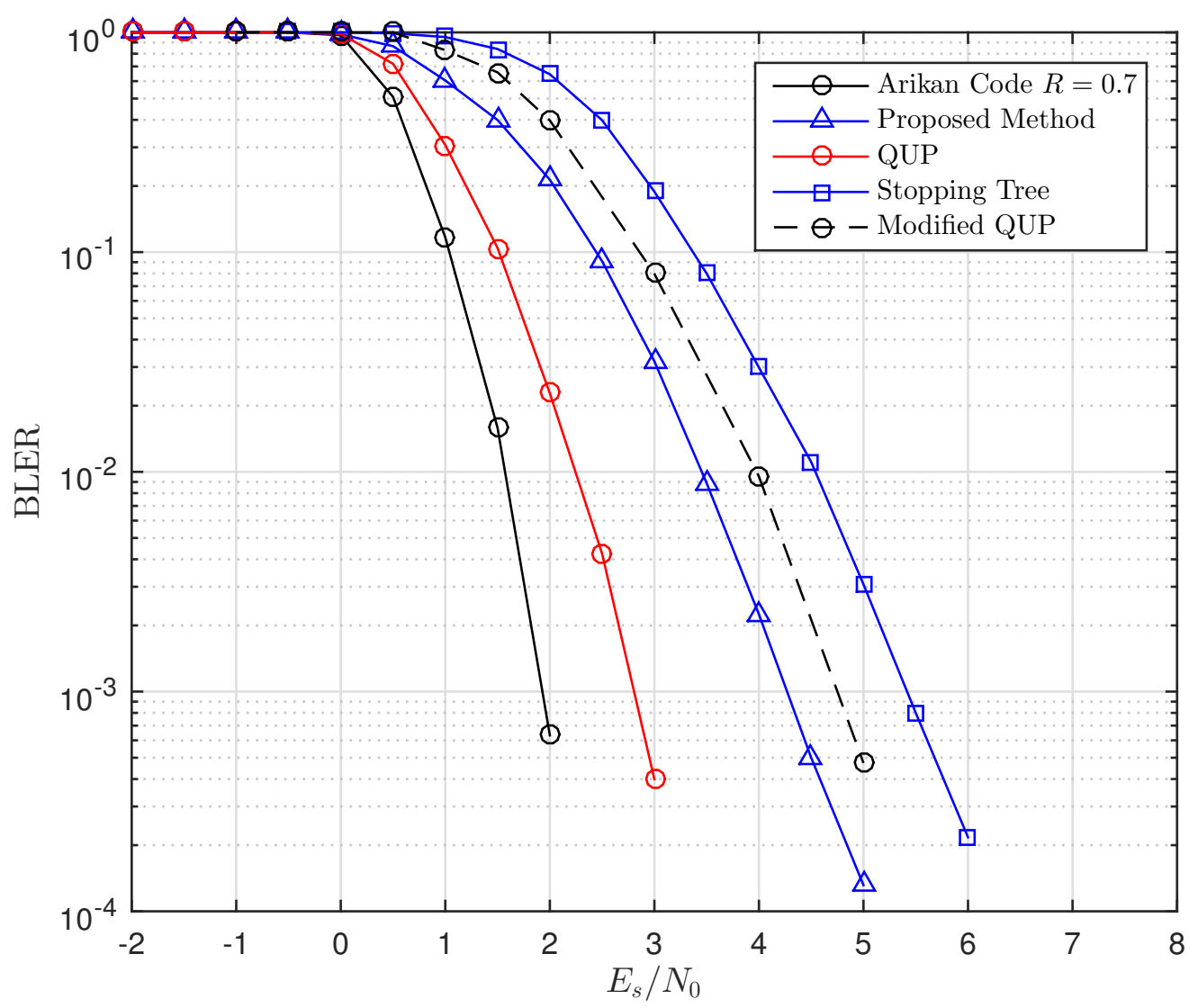

Figure 5.2: The BLERs of punctured polar codes with different puncturing algorithms.

where a mother code with a fixed frozen set is to be punctured and extended to different rates without changing the frozen set. In an attempt to find a good puncturing scheme for our system, we considered a modified QUP scheme where the transmission order of the punctured bits is determined according to QUP, but the frozen set is fixed and determined prior to puncturing. Unfortunately, as can be seen in Figure 5.2, the performance of the modified QUP is not satisfactory, so we employ our proposed puncturing algorithm in our system.

From Fig. 5.3 we can see the impact of the choice of the mother code rate on the throughput of the IR-HARQ system. Using a high-rate mother code gives superior performance at high SNRs but inferior performance at low SNRs while the converse is true for low-rate codes. To realize reasonable throughput over a wide range of SNRs it therefore seems advisable to choose $R_{M}$ to be a moderate value, such as $R_{M}=0.5$. 


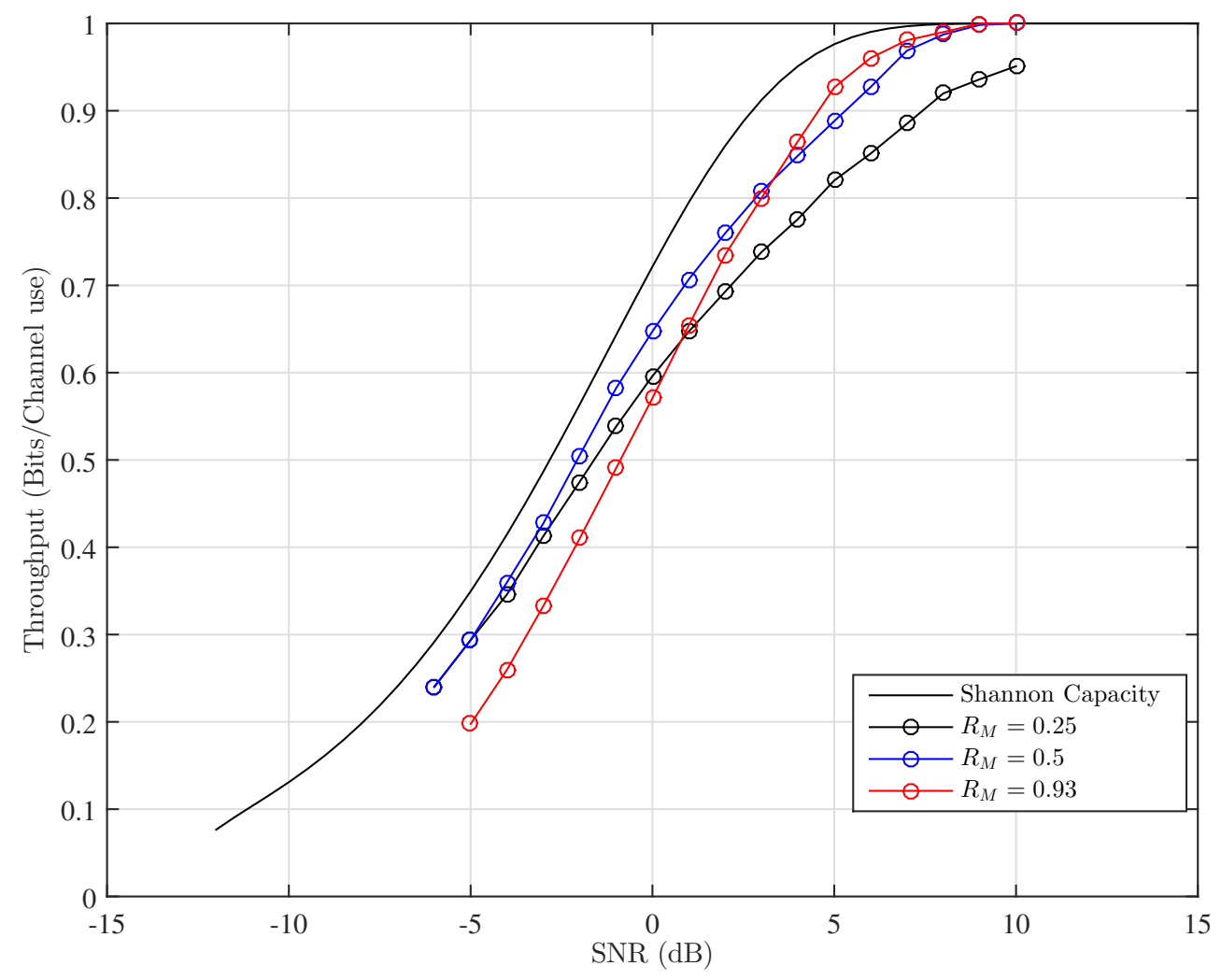

Figure 5.3: Throughput of the proposed IR-HARQ scheme with the proposed puncturing algorithm for different $R_{M}$, all with $R_{I}=R_{M}$.

It is possible to start extending before all the code bits of the mother code have been transmitted, i.e. $R_{I}>R_{M}$. Fig. 5.4 shows the effect of rate $R_{I}$ for a mother code rate of $R_{M}=0.5$. As illustrated, increasing $R_{I}$ (i.e. reducing the number of transmitted code bits before commencing extending) leads to a significant gain in throughput at high SNRs, while leading to only a very slight reduction at low SNRs. We therefore recommend using $R_{I}=1$, as arguably this gives the best overall performance. In this case the puncturing phase involves transmitting only $N_{I}=K$ code bits, and those $K$ code bits have the same indices as the $K$ information bits, $\mathcal{A}$. Once these $K$ code bits have been transmitted, the extending algorithm is used to determine the extending sequence. In theory one could start the extending phase even earlier (i.e. $R_{I}>1$ ), but this was found to be ineffective, because the code was not rate-1 decodable (which limits the throughput at very high SNRs), or degrades the 


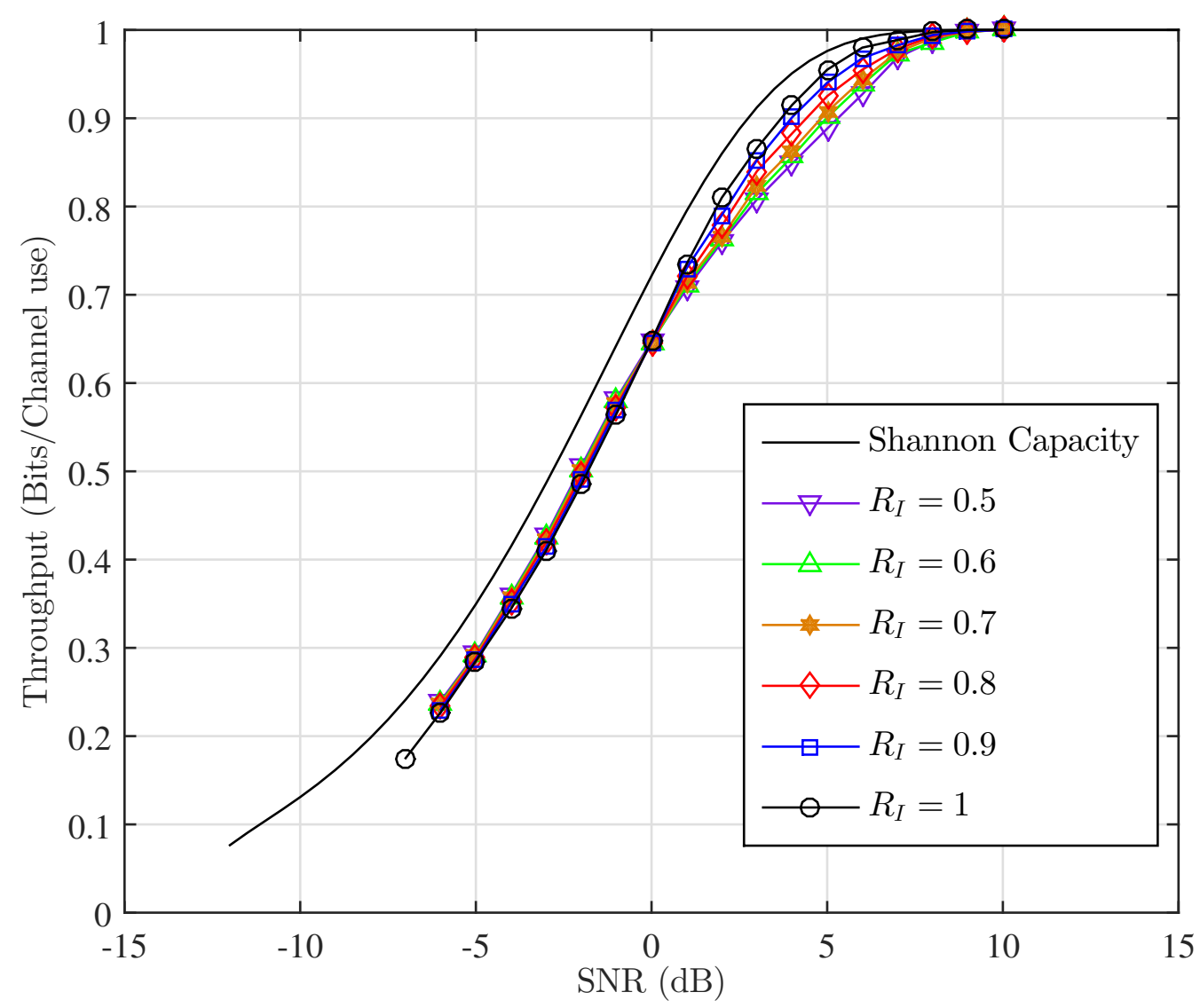

Figure 5.4: Throughput of the proposed IR-HARQ scheme with the proposed puncturing and extending algorithm with different $R_{I} \mathrm{~s}$, with $R_{M}=0.5$.

performance at low SNRs because of the greedy nature of the algorithm.

Fig. 5.5 compares the throughput of the proposed IR-HARQ scheme with parameters $\left(R_{M}, R_{I}\right)=(0.5,1)$ to the throughput of IR-HARQ schemes based on Raptor and LDPC codes. As can be seen the scheme based on Raptor codes can perform very close to the capacity at low SNRs, while leading to significant throughput drop-off at high SNRs due to non-zero overhead of the mother code. The scheme based on LDPC code, uses both puncturing and extending of an LDPC mother code of rate 8/13, similar to [24]. The mother code is punctured using the algorithm in [33] to get higher-rate codes, and is extended by adding a number of columns to the right corner of the parity check matrix of the mother code and an equal number of rows to its bottom (for more details see [24]), to get lowerrate codes. It can be seen that this scheme can get close to the capacity at intermediate SNRs. However throughput degradation occurs at both high and low SNRs. In comparison to these schemes, the proposed method can remain close to the capacity for a wider range 


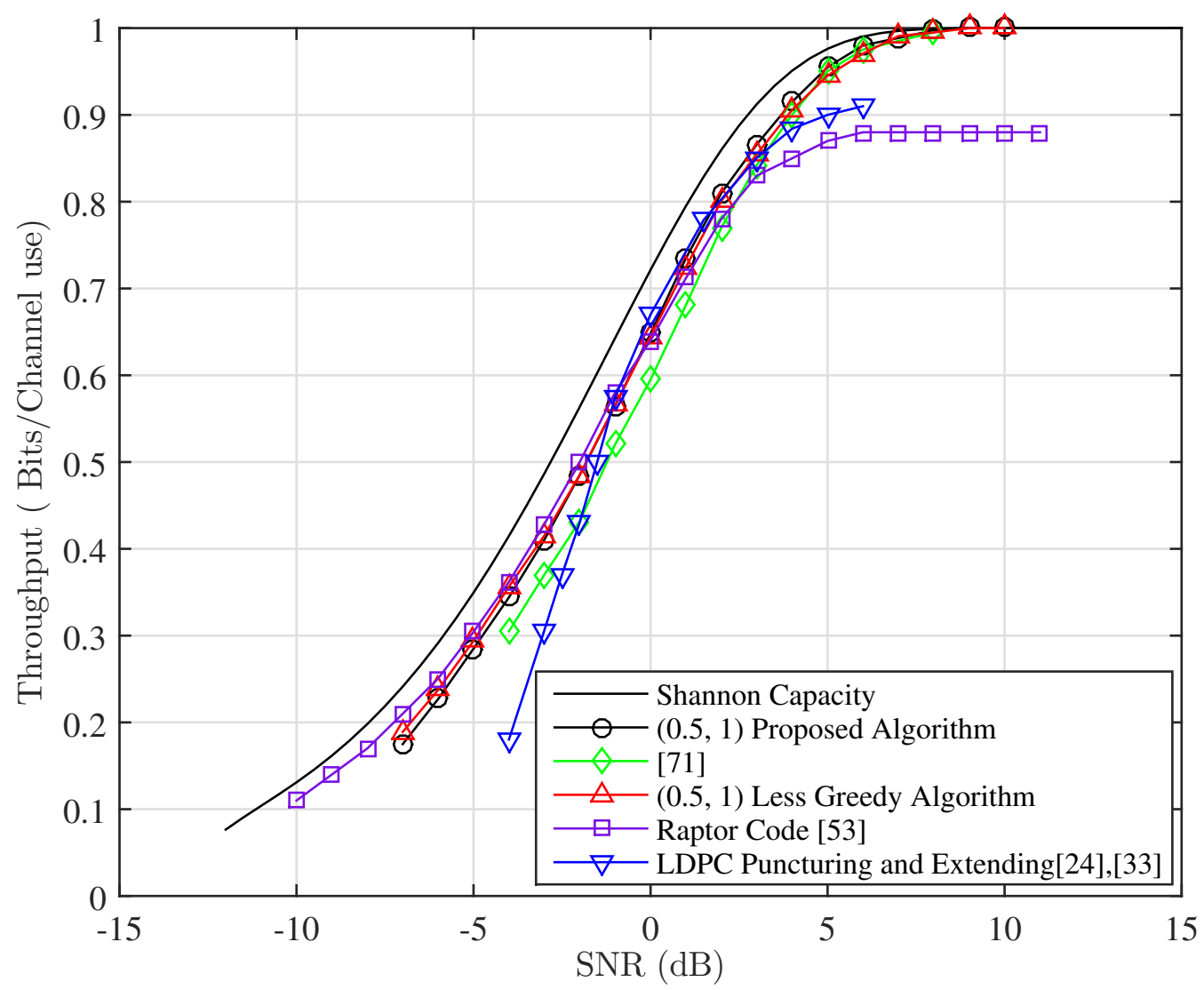

Figure 5.5: Throughput comparison of the proposed IR-HARQ scheme with other alternatives.

of SNR.

We also simulated the less greedy version of the extending algorithm. Because this algorithm is random, to measure the performance of IR-HARQ schemes constructed with this algorithm, we took 20 different realizations of the algorithm output. The average of the 20 curves was a bit worse than the proposed greedy algorithm and is not shown here. The performance of the best realization provided by the less greedy algorithm is shown in Fig. 5.5. It shows slightly lower throughput compared to the greedy algorithm at high SNRs, and a small improvement at low SNRs. Because the greedy algorithm tries to maximize the throughput with each transmitted code bit, it is very effective at high SNRs, but those decisions made early in constructing the extending sequence can lead to weaker low-rate codes. The less greedy algorithm sacrifices the high-rate performance for better low-rate performance. Which algorithm is preferable depends on the application.

Fig. 5.5 also provides a comparison of the proposed scheme to the scheme proposed in [71]. 
In the approach proposed therein, the transmitted code bits are either the code bits or the message bits. In other word, they are chosen to be either from column 0 or $n$, which makes it a special case of our proposed scheme where the code bits can be possibly chosen from all columns of the graph. As can be seen, allowing the extending nodes to be chosen from any nodes of the graph, if chosen appropriately, can result in improvements in the throughput. However, because of the differences in the choice of some system parameters between our scheme and the results in [71], a more detailed comparison is needed.

In [71], transmission is carried out in clusters of $S$ bits at a time, with decoding attempted after each cluster has been received, whereas the results for our scheme use a cluster size of $S=1$. Furthermore, in [71] the maximum number of transmitted code bits is limited to some value, $N_{\max }$, and if the message is not recovered after receiving $N_{\max }$ code bits, a transmission failure is declared and the message is retransmitted from the beginning. In our scheme no such limit is necessary, but can be implemented. The effect of $S$ and $N_{\text {max }}$ on the throughput are shown in Fig. 5.6. As can be seen, increasing $S$ from 1 to 32, while keeping $N_{\max }$ unlimited, causes a degradation in the throughput at high SNRs. This degradation is caused by the increased granularity between decoding attempts, whereby a whole cluster is transmitted even through only a few additional bits may have been needed. At low SNRs the cluster size is less important, but limiting $N_{\max }$ does have an effect. As shown in Fig. 5.6 decreasing $N_{\text {max }}$ leads to a decrease in throughput. In general, it is better to continue transmitting additional code bits than to drop a packet and restart, although practical system-level considerations generally impose some limit on $N_{\text {max }}$ eventually.

It is also worth seeing the effects of increasing the length of the mother code on the performance of the extending algorithms since polar codes are theoretically optimum only in the asymptotic sense. Using the technique in [73], we have designed mother polar codes of three different lengths: $N_{M}=1024,2048$, and 65536. Fig. 5.7 depicts the throughput of the three different codes where used with the proposed puncturing and extending algorithms. As can be seen, the $N_{M}=2048$ code is up to $0.3 \mathrm{~dB}$ better than $N_{M}=1024$ at intermediate SNRs, while this gain becomes smaller at lower SNRs. This improvement in the throughput brought by the increase in length seems to be limited as a length of 65536 does not yield significant gain over the length $N_{M}=2048$. 


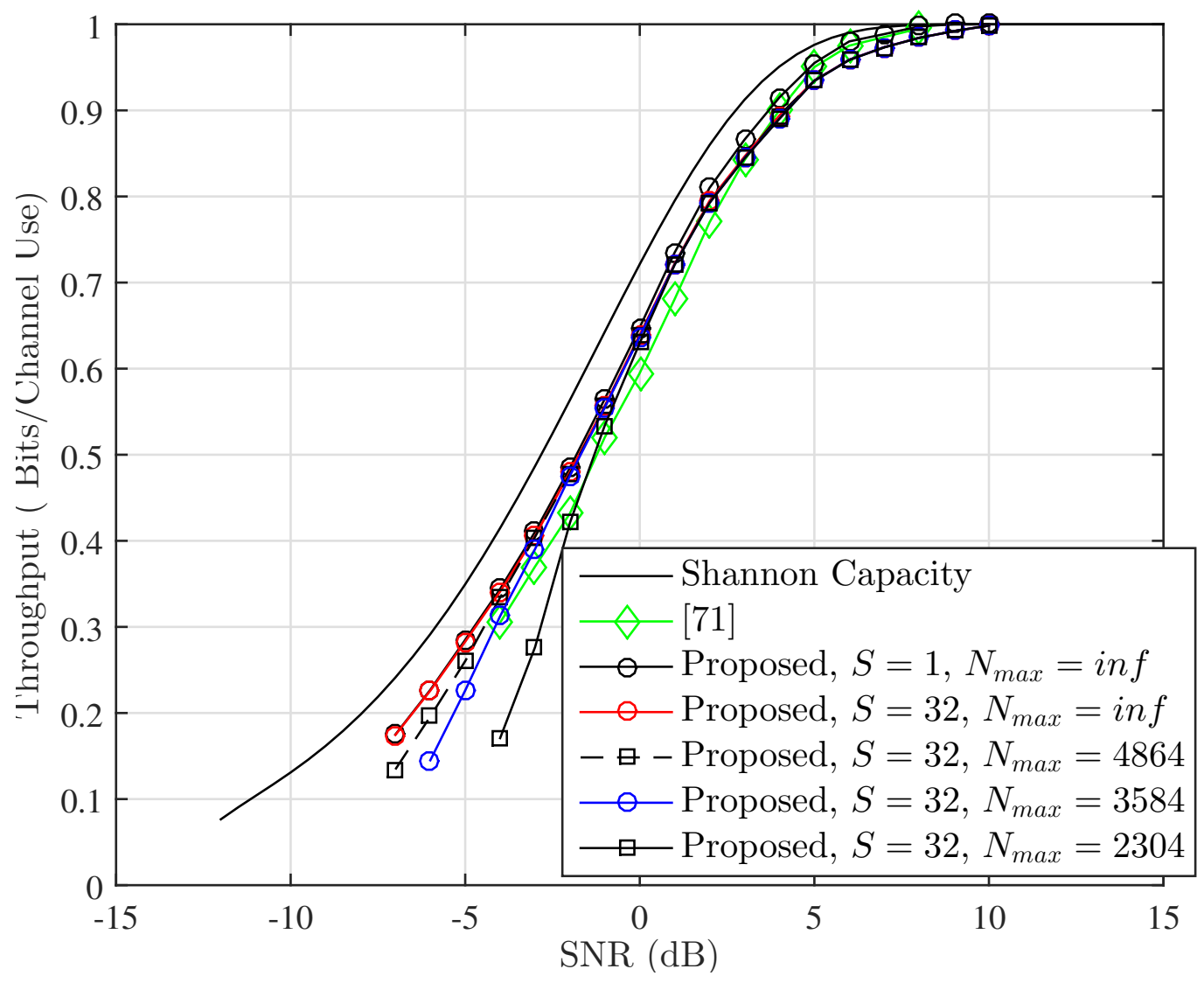

Figure 5.6: Throughput of the proposed system for different number of decodings with a cluster size of $S=32$.

\subsection{Conclusion}

We have introduced the concept of polar code extending as an effective method to construct polar codes for IR-HARQ schemes. The framework of polar code extending is quite general and includes the schemes which retransmit the code bits or the message bits and perform maximum ratio combining as a special case. It allows a possibly repeated transmission of code bits from a set of code bits of size $N_{M} \log _{2} N_{M}+K$, for a mother polar code of length $N_{M}$ with message size $K$. We proposed a throughput-specific extending algorithm to extend a possibly punctured polar code. The proposed extending method, in conjunction with the proposed puncturing method can result in a universal capacity-approaching IRHARQ scheme that can be implemented with the same encoding and decoding complexity as the original polar mother code. 


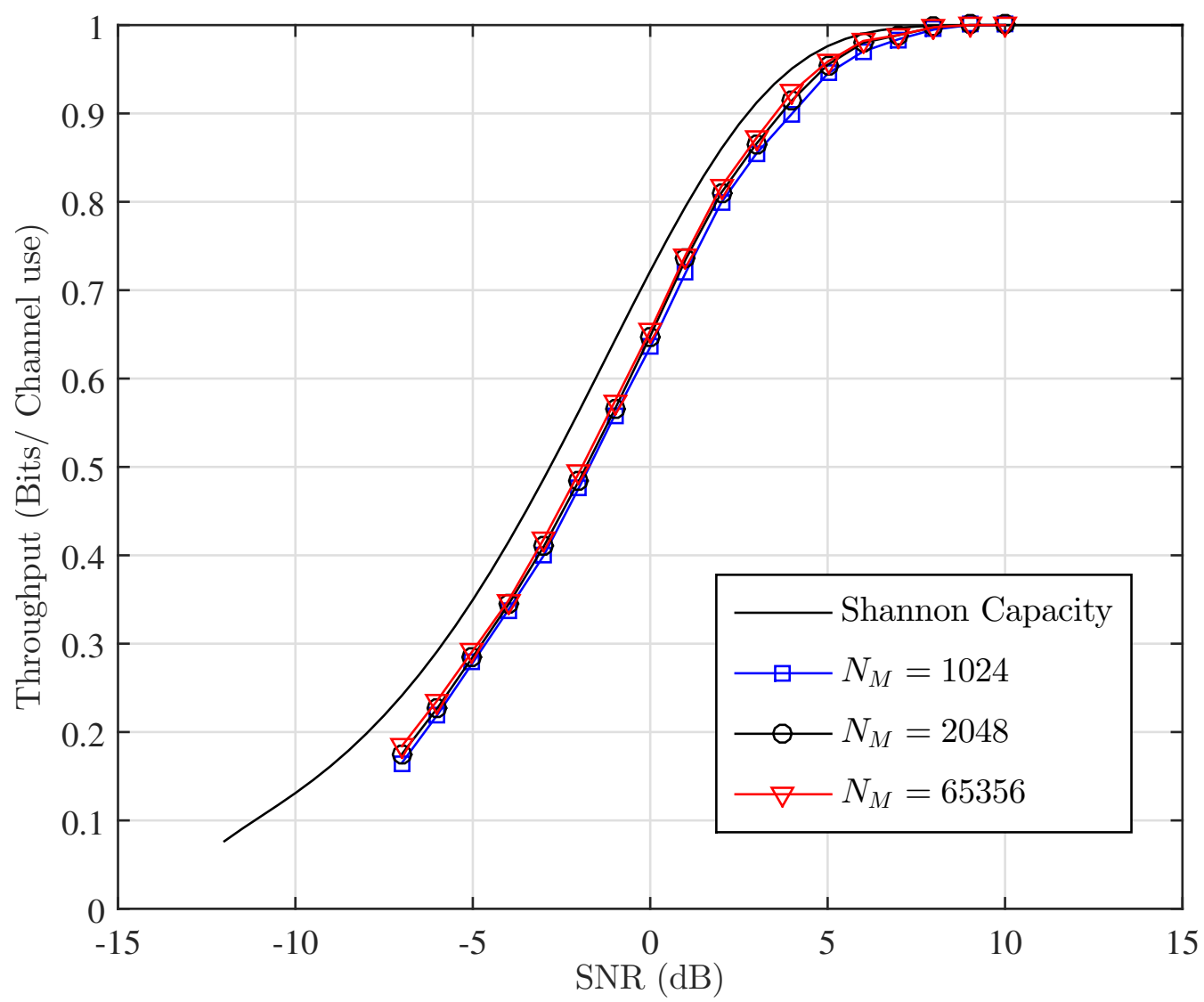

Figure 5.7: Throughput of the proposed IR-HARQ scheme for different lengths of the mother polar code, with $R_{M}=0.5$ and $R_{I}=1$. 


\section{Chapter 6}

\section{Design of Generalized Concatenated Polar Codes}

Since the performance of IR-HARQ schemes is closely related to the performance of the mother code, it is prudent to design better mother codes to be employed in the IR-HARQ scheme. Research has also been done on improving the performance of polar codes in the finite-length regime. One approach to improve the performance of polar codes is to construct polar codes with arbitrary kernel sizes which can result in a larger error exponent [80]- [81]. There have also been studies on constructing concatenated polar codes. In particular the authors in [82] proposed a concatenated code which employs polar codes and interleaved Reed-Solomon codes and reported an improvement on the performance of the conventional polar codes. A different concatenated scheme using polar and LDPC codes as the outer and inner codes is proposed in [68]. The authors in [83] proposed a modified SC decoder for polar codes by applying a short linear block code on a certain number of the least reliable information bits and a certain number of frozen bits and modified the SC decoder accordingly. Performance improvements were reported with the modified SC decoder. As another approach to improve the performance of polar codes at finite length, a different concatenation method has been proposed which is based on the multilevel nature of polar codes. In particular, it is shown that the polar codes can be considered as a special class of generalized concatenated codes (GCC) with the outer and inner codes being polar codes themselves. GCC codes based on polar codes (GCC-polar codes) have been proposed by replacing the outer codes of the polar codes with some arbitrary outer codes under decoders other than SC decoding [84]- [86]. In [86], the authors proposed a GA method to design the GCC-polar codes. They used GA to design the outer codes for an ML decoder. Once the outer codes were designed, they proposed a rate allocation algorithm to design the GCC-polar codes. Performance improvement over the conventional polar codes were reported with an increase in the decoding complexity of the outer codes from $O(L \log L)$ 
to $O\left(L^{t+1}\right)$, where $L$ is the length of the outer codes, and $t$ is some reprocessing order for decoding the outer codes. In this chapter we will follow this line of work. Similar to [86], our work focuses on designing GCC-polar codes. However, this work is different from [86] in the following ways:

a) In [86] the outer codes are designed for transmission over an AWGN channel while the equivalent channel seen by each outer code is not AWGN. In this paper we design the outer codes for the actual channel seen by them. The channel for each outer code is described by a transition LLR density obtained via density evolution. An upper bound for the block-wise ML decoding of the outer code over an arbitrary BIMOS channel is used to design the outer codes.

b) Once a set of outer codes with different dimensions are obtained, the GCC-polar code design invokes a rate allocation algorithm to determine the rates of the outer codes to use. The author in [86] proposed a heuristic equal error probability rule algorithm to allocate rates to the outer codes with an attempt to make their resultant decoding error probabilities as equal as possible. In this work we propose a rate allocation algorithm with the goal of minimizing the BLER of the GCC-polar code. The overall BLER of the GCC-polar code is described in terms of the BLERs of the outer codes and the rates of the outer codes are chosen to minimize the overall BLER.

c) Unlike [86] we aim to decrease the overall decoding complexity of the GCC codes. To this end we only design very short outer codes $(L \leq 8)$. The overall decoding complexity analysis for a given GCC-polar code is presented. The complexity is shown to be a function of the outer code rate distribution of the GCC code. Rate distributions with lower overall complexity and superior performance are found.

The rest of the chapter is organized as follows. In Section 6.1 we define the outer codes of a polar code. In Section 6.2 we present the proposed method for designing GCC-polar codes. We present a channel-specific method to design the outer codes based on DE to minimize an upper bound on their BLER under ML decoding. The output of this step is a bank of outer codes of different rates. We then present a rate allocation algorithm to determine the rates of the outer codes to be used for the GCC-polar code. A complexity analysis is also given in this section. Section 6.3 presents performance evaluation results based on computer simulation, comparing some designed GCC-polar code with the conventional one. The chapter is concluded in Section 6.4. 


\subsection{Outer codes of a Polar Code}

The PC graph makes it possible to visualize smaller outer codes within the polar code. Consider a fixed polar code of length $N=2^{n}$ with information set $\mathcal{A}$ of cardinality $K$. The outer codes of length $L=2^{l}$ for $l \in\{0, \ldots, n\}$ of this polar code can be described as follows. The set $\mathcal{A}$ induces a set of frozen and information bits at the first column of the PC graph. The values of all frozen bits are assumed to be zero. For $k \in\{1, \ldots, N / L\}$ let $\mathcal{S}_{k}=\{k L-L+1, \ldots, k L\}$ and $\mathcal{A}_{k}$ be the set containing the information bit indices from $\mathcal{S}_{k}$, i.e. $\mathcal{A}_{k}=\mathcal{A} \cap \mathcal{S}_{k}$. Also let $\omega_{k}=\left|\mathcal{A}_{k}\right|$ for $k=1, \ldots, N / L$. The $k$-th length- $L$ outer code of the polar code, labelled $C_{L, k}$ in Fig. 6.1, is then an $\left(L, \omega_{k}\right)$ linear block code which is defined as the mapping between the message bits $u_{i}, i \in \mathcal{A}_{k}$ and the code bits $v_{i, l}, i \in \mathcal{S}_{k}$. An outer code with $\omega_{k}=0$ is a code whose code bits are always zeros.

As an example consider the length $N=8$ polar code of rate $1 / 2$ with the information set $\mathcal{A}=\{4,6,7,8\}$ whose PC graph is depicted in Fig. 6.1. We have $u_{i}=0$ for $i \in\{1,2,3,5\}$. The outer codes of length $L=1,2,4$ are specified by the dashed squares in the graph. For outer code length $L=1$, the parts of the graph corresponding to these codes are specified by the dashed squares including just one row of the graph. These eight outer codes are the mappings between $u_{k}$ and $v_{k, 0}$ for $k=1, \ldots, 8$ and are of rates $0,0,0,1,0,1,1$ and 1 respectively. For $L=2$, the outer codes are specified by the dashed squares including two rows of the graph. There are four of these codes which are of rates $0,1 / 2,1 / 2$ and $2 / 2$, respectively. The first outer code is of rate 0 , which maps $\left(u_{1}=0, u_{2}=0\right)$ to the code bits $\left(v_{1,1}=0, v_{2,1}=0\right)$. This code always generates the all-zero codeword. The second outer code is of rate $1 / 2$, mapping $\left(u_{3}=0, u_{4}\right)$ to $\left(v_{3,1}, v_{4,1}\right)$. The third one is also of rate $1 / 2$, mapping $\left(u_{5}=0, u_{6}\right)$ to $\left(v_{5,1}, v_{6,1}\right)$. Finally the fourth outer code is of rate $2 / 2$ and maps $\left(u_{7}, u_{8}\right)$ to $\left(v_{7,1}, v_{8,1}\right)$. For $L=4$, there are two outer codes, specified by the dashed squares containing 4 rows of the graph. The first code is of rate $1 / 4$ and maps $\left(u_{1}=0, u_{2}=0, u_{3}=0, u_{4}\right)$ to $\left(v_{1,2}, v_{2,2}, v_{3,2}, v_{4,2}\right)$. The second code is of rate $3 / 4$ and maps $\left(u_{5}=0, u_{6}, u_{7}, u_{8}\right)$ to $\left(v_{5,2}, v_{6,2}, v_{7,2}, v_{8,2}\right)$. For $L=8$, there is only one outer code which is in fact the polar code itself, mapping $\left(u_{1}=0, u_{2}=0, u_{3}=0, u_{4}, u_{5}=0, u_{6}, u_{7}, u_{8}\right)$ to $\left(v_{1,3}, \ldots, v_{8,3}\right)$.

For a given polar code of length $N$ with an associated outer code length $L=2^{l}$, number the outer codes from top to bottom of the PC graph as 1 to $N / L$. The SC decoder calculates the LLRs $\lambda_{i, l}$ for $i \in \mathcal{S}_{k}$, and uses them to perform SC decoding of the $k$-th outer code to recover its $\omega_{k}$ message bits. Once these message bits are decoded the estimated code bits 


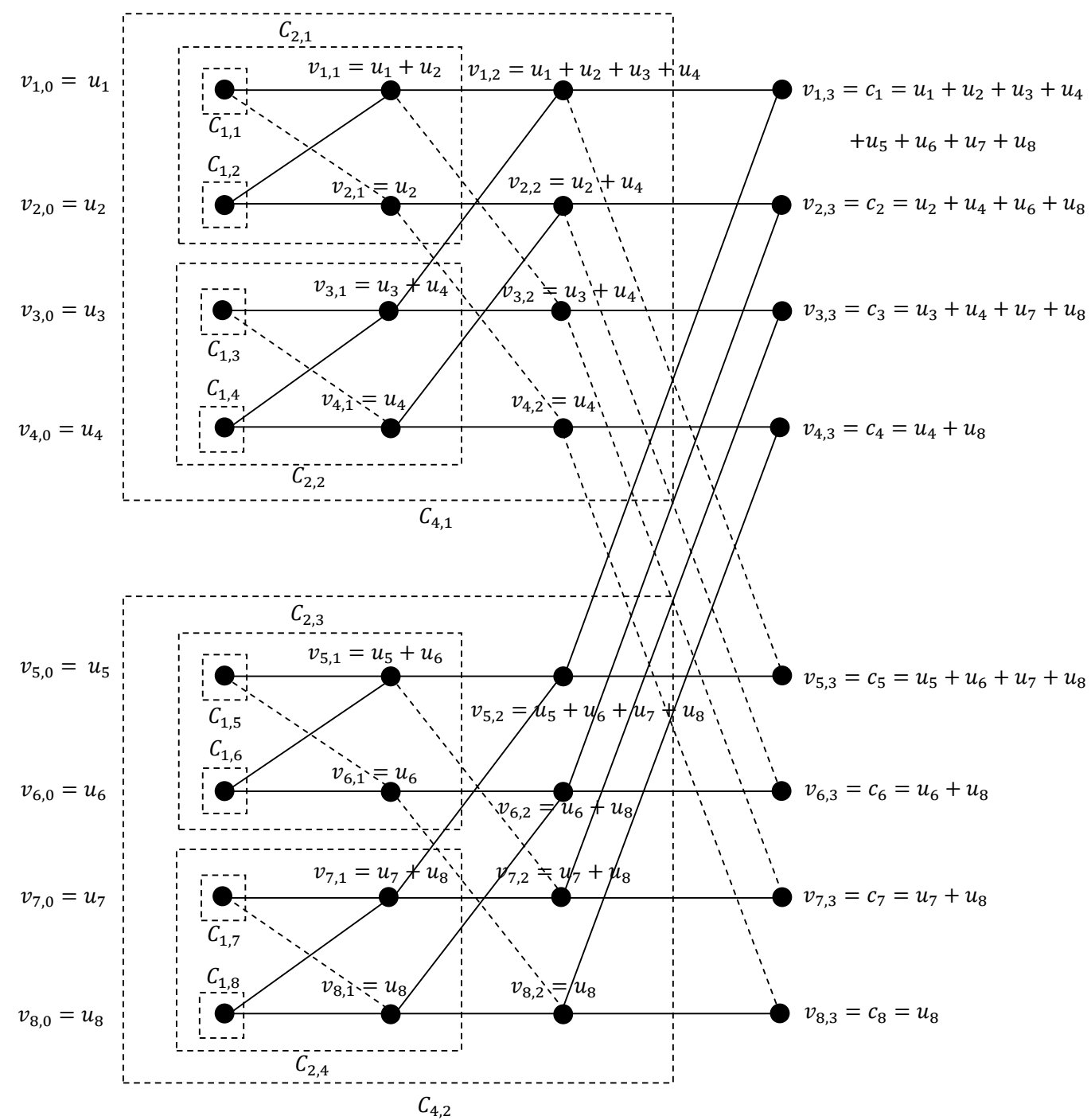

Figure 6.1: The PC graph of the polar code of length $N=8$ and its outer codes of lengths $L=1,2$, and 4 .

$\hat{v}_{i, l}$ for $i \in \mathcal{S}_{k}$ are calculated using the same procedure as the encoder and the SC decoding process continues. As we will see later the performance of a polar code depends on the performance of its outer codes to a significant extent.

\subsection{Proposed Method for Designing GCC-Polar Codes}

To improve the performance of polar codes, the GCC-polar code is proposed by modifying the PC graph of the polar code of length $N=2^{n}$ by replacing the outer codes of length 

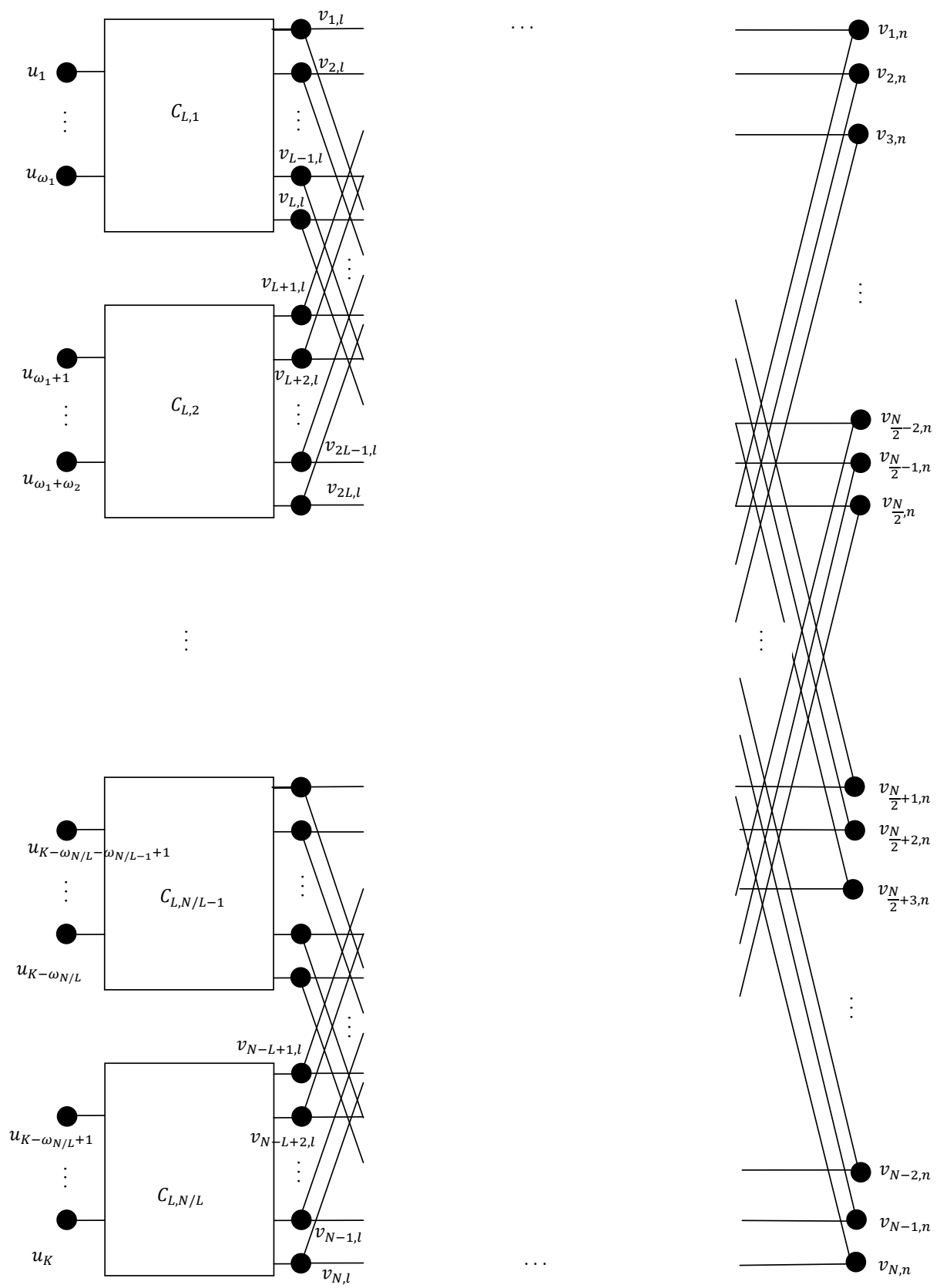

Figure 6.2: The encoding and decoding graph of a GCC-polar code of length $N=2^{n}$ with a set of $\left(L=2^{l}, \omega_{k}\right)$ outer codes $C_{L, k}$.

$L=2^{l}$ with better linear block codes [86]. Fig. 6.2 depicts a graphical representation of the GCC-polar code. The outer codes $C_{L, k}$ can be arbitrary linear block codes of rates $\omega_{k} / L$ for $k=1, \ldots, N / L$. The GCC-polar code is then an $(N, K)$ code where $K=\sum_{k=1}^{N / L} \omega_{k}$. The part 
of the graph that includes the nodes $v_{i, l}, i=1, \ldots, N$ and the nodes and edges connected to them from the right is identical to the PC graph of a polar code of length $N$ obtained by removing all the nodes at columns 0 to $l-1$ and the edges connected to them.

Encoding is carried out on this modified graph by applying the $K$ message bits to the graph in Fig. 6.2. Each outer code $C_{L, k}$ takes its $\omega_{k}$ input message bits and calculates the code bits $v_{i, l}, i \in \mathcal{S}_{k}$. Note that in the case of rate-0 outer codes, the output code bits will be all zeros. Once the values of the code bits $v_{i, l}$ are calculated, the rest of the encoding process is exactly the same as for the conventional polar codes because the graph to the right of the outer codes is the same as the PC graph including columns $l$ to $n$. Using this procedure the values of all the nodes $v_{i, j}, i=1, \ldots, N, j=l, \ldots, n$ are calculated which includes the code bits $c_{i}=v_{i, n}$ to transmit over the channel. These code bits are then transmitted over the channel $\mathcal{W}$ and the values $y_{i}$ are received for each code bit $c_{i}$.

The decoding of the GCC-polar code is carried out as follows. The channel LLRs, $\lambda\left(y_{i}\right)=$ $\ln \frac{f_{W}\left(y_{i} \mid 0\right)}{f_{W}\left(y_{i} \mid 1\right)}$ for code bit $c_{i}, i=1, \ldots, N$, are calculated and applied to the rightmost column, so $\lambda_{i, n}=\lambda\left(y_{i}\right)$. These LLRs propagate leftwards through the graph in the same way as for the SC decoder until the LLRs for the code bits of outer code $C_{L, 1}$ (i.e. the LLRs $\lambda_{i, l}, i \in \mathcal{S}_{1}$ ) are calculated. At this point the decoding process is taken over by a separate decoder for outer code $C_{L, 1}$. The outer code decoder performs the decoding and recovers its message and code bits. Once $\hat{v}_{i, l}, i \in \mathcal{S}_{1}$ are calculated, the decoding process continues in the same way as the SC decoder until the LLRs for the code bits of the next outer code are calculated, when the decoder for that outer code is invoked. This process continues until all the outer codes are decoded and thus all the message bits are recovered. In this paper we consider block-wise ML decoding for the outer codes. An ML decoding rule for a linear block code using the LLRs of its code bits is presented in the Appendix 8.0.1.

\subsubsection{Design of outer codes}

Similar to conventional polar codes where the BLER can be expressed in terms of the firsterror event probabilities of the bit-channels $P_{e}(i)$, the BLER, $P_{B}$, of the GCC-polar code can be estimated as a function of the BLERs of the outer codes given that the previous outer codes have been decoded correctly. Let $P_{L, k}$ be the first-block-error probability of outer code $C_{L, k}$ (i.e., the BLER of $C_{L, k}$ given that all the codes $C_{L, j}, j=1, \ldots, k-1$ have been decoded correctly). Also let $\mathcal{E}_{k}$ be the event that the decoding of outer code code $C_{L, k}$ fails, so 


$$
P_{L, k}=\operatorname{Pr}\left(\mathcal{E}_{k} \mid \bigcap_{j=1}^{k-1} \overline{\mathcal{E}_{j}}\right) .
$$

The BLER of the GCC-polar code is equal to the probability that at least one of the outer codes will have a decoding failure, so

$$
\begin{aligned}
P_{B} & =\operatorname{Pr}\left(\bigcup_{k=1}^{N / L} \mathcal{E}_{k}\right)=1-\operatorname{Pr}\left(\bigcap_{k=1}^{N / L} \overline{\mathcal{E}_{k}}\right) \\
& =1-\prod_{k=1}^{N / L} \operatorname{Pr}\left(\overline{\mathcal{E}_{k}} \mid \bigcap_{j=1}^{k-1} \overline{\mathcal{E}_{j}}\right) \\
& =1-\prod_{k=1}^{N / L}\left(1-P_{L, k}\right)
\end{aligned}
$$

As can be seen from (6.2) the first-block-error probability $P_{L, k}$ of outer code $C_{L, k}$ plays an important role in the overall performance of the GCC-polar code. For a given choice of $\omega_{k}, C_{L, k}$ should be designed to minimize its BLER. That is, for each $k \in\{1, \ldots, N / L\}$, we need to find the best $\left(L, \omega_{k}\right)$ linear block code to use for $C_{L, k}$ in Fig. 6.2. However, as will be discussed in Section 6.2.2, it is more useful to find the best $(L, \omega)$ codes for all $\omega \in$ $\{1, \ldots, L\}$ instead of just for one given $\omega=\omega_{k}$. Assuming that the all-zero codeword was transmitted and the codewords of the previous outer codes are known to the decoder, the density of the LLR $\lambda_{i, l}$ for $i \in \mathcal{S}_{k}$ calculated at output node $(i, l)$ of this outer code is $f_{i, l}(\lambda)$, which can be obtained by DE. According to the way that these densities are calculated they are all identical, so $f_{i, l}(\lambda)=f_{k L, l}(\lambda)$ for all $i \in \mathcal{S}_{k}$. We therefore design $C_{L, k}$ for transmission over a BIMOS channel whose LLR-transition density for a zero input bit is $f_{k L, l}(\lambda)$. Since we are performing block-wise ML decoding of $C_{L, k}$, its decoding performance does not depend on the mapping between the message words and the codewords but only on its code space. So without loss of generality we can assume that the generator matrix $\mathbf{G}_{\omega}^{(k)}$ is in systematic form, so $\mathbf{G}_{\omega}^{(k)}=\left[\begin{array}{ll}\mathbf{I}_{\omega} & \mathbf{P}_{\omega}^{(k)}\end{array}\right]$ where $\mathbf{P}_{\omega}^{(k)}$ is a $\omega \times(L-\omega)$ matrix. The ML decoding performance of this code is mainly determined by the weight distribution of the code. Let $A_{d}$ be the weight distribution of this outer code, where $A_{d}$ denotes the number of codewords of weight $d$. The BLER of $C_{L, k}$ over the considered BIMOS channel can then 
be upper bounded by

$$
P_{L, k} \leq \sum_{d=1}^{L} A_{d} \gamma_{d}^{(k)}
$$

where

$$
\gamma_{d}^{(k)}=\int_{-\infty}^{0} f_{k L, l}^{\otimes d}(\lambda) d \lambda
$$

where $f_{k L, l}^{\otimes d}(\lambda)$ is the convolution of $f_{k L, l}(\lambda)$ with itself $d$ times. A proof of (6.3) is given in the Appendix.

To design an $(L, \omega)$ code for $C_{L, k}, \mathrm{DE}$ is performed to calculate the density $f_{k L, l}(\lambda)$. Then, the parameters $\gamma_{d}^{(k)}$ for $d=1$ to $L$ are calculated from (6.4). We then look for $\mathbf{P}_{\omega}^{(k)}$ such that the resultant $A_{d}$ minimizes the upper bound in (6.3). The search space for this optimization problem has a size of $2^{\omega .(L-\omega)}$. For very short outer code lengths, $L$, one can perform an exhaustive search. However as $L$ gets larger the search space grows exponentially and an exhaustive search becomes infeasible. In this case one may resort to sub-optimum methods to construct the outer codes. It is noteworthy that in our work for the purpose of decoding complexity we have considered very short outer codes $(L \leq 8)$ for which both the search space is small and ML decoding can be performed with low complexity. As we will see the resultant short outer codes are nonetheless effective and provide noticeable coding gain over the conventional polar codes. At these code lengths it is possible to perform an exhaustive search rather than a partial suboptimum optimization as proposed in [87]- [88]. Nevertheless it should be noted that the advantage of the methods from [87]- [88] is that unlike our proposed optimization it can be used to optimize larger outer codes for use with a low-complexity decoder. However since the purpose of this work is to improve the performance of the Arikan code while reducing the complexity, only very short outer codes have been considered for which the exhaustive search for the optimized generator matrices is computationally possible. Moreover, unlike [87]- [88], the exhaustive search optimizes the generator matrices for the specific channel seen by the outer code, which is an arbitrary BIMOS channel described by the LLR density at the nodes at column $l$ of the PC graph.

As an example of an application of this design process, we found the optimum codes for all $\omega \in\{1, \ldots, L\}$ and $k \in\{1,2, \ldots, N / L\}$ for the case of $L=8$ and $N=1024$. The underlying channel $\mathcal{W}$ is a BI-AWGN channel with binary phase shift keying (BPSK) at $E_{s} / N_{0}=0 \mathrm{~dB}$. Although each outer code will experience a different equivalent BIMOS 
channel depending on its position in the PC graph, we found that the optimum code for a given $\omega$ did not depend on $k$. In general this may not be the case but it does seem likely that a code that is optimal for some $k$ will be near-optimal for all $k$, allowing us to implement only $L$ different codes, instead of $L \times N / L=N$. We stress that the optimal design of the outer codes will consist of $L$ possibly different generator matrices of different rates for each outer code $C_{L, 1}, \ldots, C_{L, N / L}$. The generator matrices that we found for $L=8$ to minimize (6.3) are $\mathbf{G}_{\omega}^{(k)}=\left[\begin{array}{ll}\mathbf{I}_{\omega} & \mathbf{P}_{\omega}^{(k)}\end{array}\right]$ where

$$
\begin{aligned}
& \mathbf{P}_{8}^{(k)}=\emptyset, \mathbf{P}_{7}^{(k)}=\left[\begin{array}{lllllll}
1 & 1 & 1 & 1 & 1 & 1 & 1
\end{array}\right]^{T} \\
& \mathbf{P}_{6}^{(k)}=\left[\begin{array}{llllll}
0 & 1 & 1 & 1 & 0 & 1 \\
1 & 0 & 1 & 1 & 1 & 1
\end{array}\right]^{T} \\
& \mathbf{P}_{5}^{(k)}=\left[\begin{array}{lll}
1 & 1 & 1 \\
1 & 1 & 0 \\
0 & 1 & 1 \\
0 & 1 & 0 \\
1 & 0 & 1
\end{array}\right], \mathbf{P}_{4}^{(k)}=\left[\begin{array}{llll}
0 & 1 & 1 & 1 \\
1 & 0 & 1 & 1 \\
1 & 1 & 0 & 1 \\
1 & 1 & 1 & 0
\end{array}\right] \\
& \mathbf{P}_{3}^{(k)}=\left[\begin{array}{lllll}
0 & 1 & 1 & 1 & 1 \\
1 & 0 & 1 & 1 & 1 \\
1 & 1 & 0 & 1 & 0
\end{array}\right], \mathbf{P}_{2}^{(k)}=\left[\begin{array}{llllll}
1 & 0 & 1 & 0 & 1 & 1 \\
0 & 1 & 0 & 1 & 1 & 1
\end{array}\right] \\
& \mathbf{P}_{1}^{(k)}=\left[\begin{array}{lllll}
1 & 1 & 1 & 1 & 1 \\
1 & 1
\end{array}\right]
\end{aligned}
$$

where $\varnothing$ is the null matrix (i.e. $\mathbf{G}_{8}^{(k)}$ is the identity matrix).

Fig. 6.3 shows the BLER performance of these codes over a BI-AWGN channel, for rates from $1 / 8$ to $8 / 8$. For comparison, we have also shown the performance of Arikan's $L=8$ outer codes. The generator matrices of Arikan's outer codes are constructed by removing rows in the order of $1,2,3,5,4,6,7,8$. That is, the outer codes of rate $8 / 8$ uses the full $\mathbf{G}_{8}$, that of rate $7 / 8$ uses $\mathbf{G}_{8}$ with row 1 removed, rate $6 / 8$ with rows 1 and 2 removed, 5/8 with 


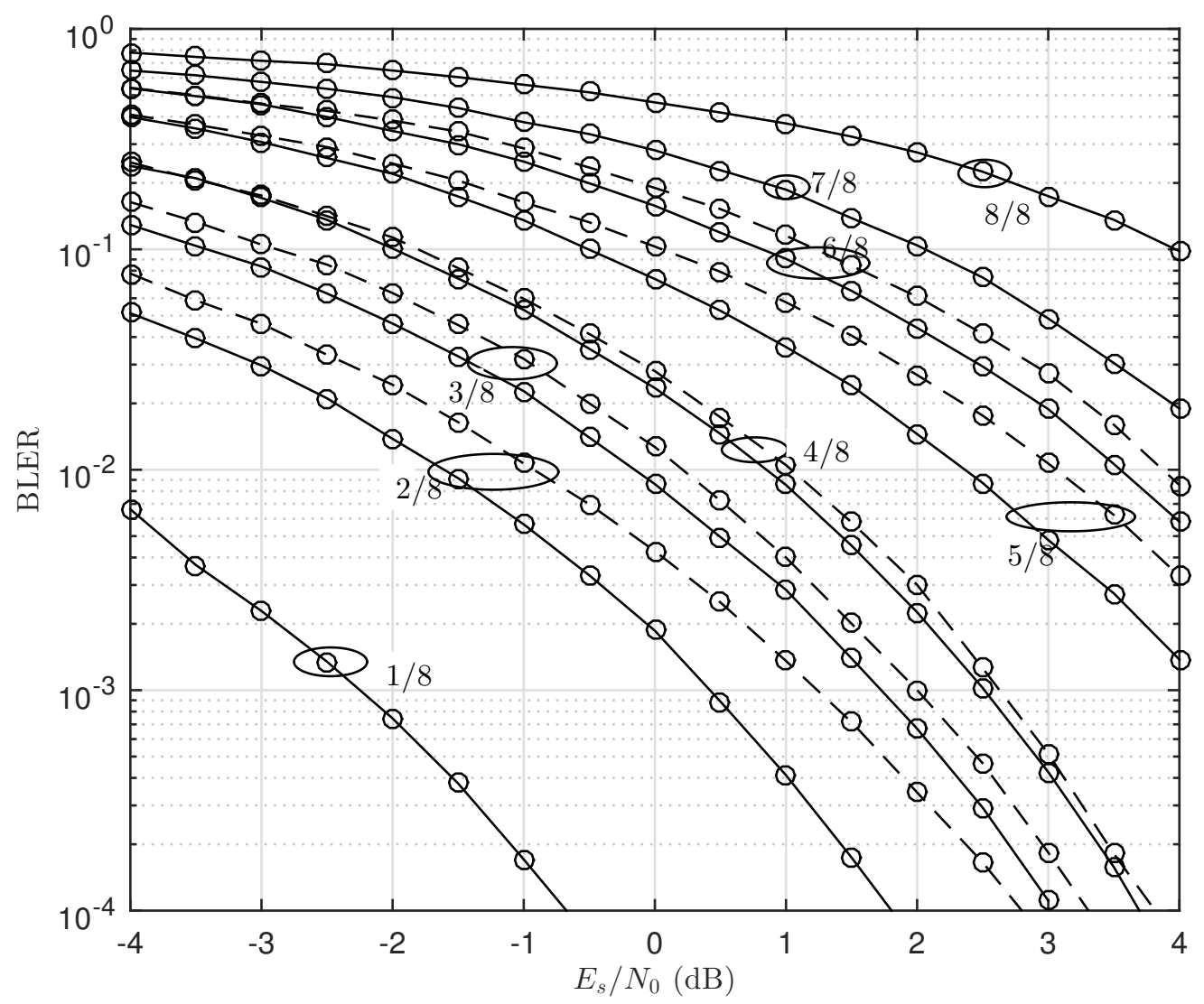

Figure 6.3: The performance of the designed outer codes (solid lines) versus Arikan's (dashed lines) over the BI-AWGN channel, for different code rates.

rows 1, 2 and 3 removed, 4/8 with rows 1,2,3 and 5 removed and so on. As can be seen in Fig. 6.3, the designed outer codes outperform Arikan's at all rates except for 1/8, 7/8 and $8 / 8$, where they tie. The reason the results tie for these rates is that the designed outer codes and the Arikan's codes are the same codes. For example at rates $1 / 8,7 / 8$ and $8 / 8$ both codes are the repetition code, the single-parity check code and the rate- 1 code, respectively. Although they use different mappings between the message words and the codewords, they will have the same BLERs. Since the designed outer codes generally outperform Arikan's codes, we expect the GCC-polar code with these outer codes to potentially outperform Arikan's polar code.

Once a "bank" of generator matrices $\mathbf{G}_{\omega}^{(k)}$ are found for $k \in\{1, \ldots, N / L\}$ and $\omega \in\{1, \ldots, L\}$, and given a choice of $\omega_{1}, \ldots, \omega_{N / L}$, the GCC-polar code in Fig. 6.2 is completely determined by placing a code with the generator matrix $\mathbf{G}_{\omega_{k}}^{(k)}$ in place of $C_{L, k}$. The performance 
of the GCC-polar code depends on the specific choices of $\omega_{k}$. In the following we focus on finding good choices of $\omega_{k}$ for a given bank of generator matrices. We refer to this process as the rate allocation process.

\subsubsection{Rate allocation algorithm}

The goal of rate allocation is to find the dimensions of the outer codes, i.e $\left(\omega_{1}, \ldots, \omega_{N / L}\right)$, to design an $(N, K)$ GCC-polar code. Let $P_{L, k}(\omega)$ be the first-block-error probability of $C_{L, k}$ if it has the generator matrix $\mathbf{G}_{\omega}^{(k)}$. A good estimation of $P_{L, k}(\omega)$ can be found by finding $\gamma_{d}^{(k)}$ using (6.4) and then calculating the upper bound in (6.3) with the weight distribution of $\mathbf{G}_{\omega}^{(k)}$ in place of $A_{d}$. Obviously, $P_{L, k}(0)=0$ for an outer code code of rate 0 . Once the first-block-error probabilities are found for $k \in\{1, \ldots, N / L\}$ and $\omega \in\{0, \ldots, L\}$, we choose $\left(\omega_{1}, \ldots, \omega_{N / L}\right)$ in such a way that (6.2) is minimized. The optimal solution to the rate allocation problem is

$$
\left(\omega_{1}^{*}, \ldots, \omega_{N / L}^{*}\right)=\underset{\left(\omega_{1}, \ldots, \omega_{N / L}\right)}{\operatorname{argmin}}\left(1-\prod_{k=1}^{N / L}\left[1-P_{L, k}\left(\omega_{k}\right)\right]\right)
$$

subject to $\sum_{k} \omega_{k}=K$ and $\omega_{k} \in\{0, \ldots, L\}$ for $k=1, \ldots, N / L$.

The search space for this optimization problem grows exponentially with $N$ and thus cannot be tackled by a brute force search. Instead we propose a computationally feasible but still optimal solution for the problem. We will prove the optimality of our solution by induction. Particularly, if one shows that if the proposed algorithm is given the optimal rate allocation for all the outer codes of a certain length, it gives the optimal rate allocation for all the outer codes twice as long, then one can use induction to prove the optimality of the algorithm. Since the optimal rate-allocation for all the outer codes of length 1 is trivial, it remains to prove that the algorithm can give the optimal rate allocation as the outer code lengths are doubled. Assume that optimal rate allocation has been performed for all outer codes of length $L^{\prime}$ for some $L^{\prime} \geq L$. For $\omega \in\left\{0, \ldots, L^{\prime}\right\}$ and $k \in\left\{1, \ldots, N / L^{\prime}\right\}, C_{L^{\prime}, k}(\omega)$, which is the $k^{\text {th }}$ outer code of length $L^{\prime}$ and rate $\omega / L^{\prime}$, is defined as optimal if it is constructed from outer codes $C_{L, j}\left(\omega_{j}^{*}\right)$ for $j \in\left\{(k-1) L^{\prime} / L+1, \ldots, k L^{\prime} / L\right\}$ when $\left\{\omega_{j}^{*}\right\}$ is chosen to minimize the first-block-error probability of $C_{L^{\prime}, k}(\omega)$. Let $\alpha_{L^{\prime}, k}(\omega)$ be a vector of length $L^{\prime} / L$ containing the optimal rates of the outer codes of length $L$ of $C_{L^{\prime}, k}(\omega)$, and let $P_{L^{\prime}, k}(\omega)$ be the first-block-error probability of $C_{L^{\prime}, k}(\omega)$.

Now consider the outer codes of length $2 L^{\prime}$ constructed by combining the codewords 
of $C_{L^{\prime}, k}\left(\omega_{k}\right)$ and $C_{L^{\prime}, k+1}\left(\omega_{k+1}\right)$ for any odd $k \in\left\{1, \ldots, N / L^{\prime}-1\right\}$. This outer code, $C_{2 L^{\prime},(k+1) / 2}\left(\omega_{k}+\omega_{k+1}\right)$, will have rate $\left(\omega_{k}+\omega_{k+1}\right) / 2 L^{\prime}$ and a first-block-error probability of $1-\left[1-P_{L^{\prime}, k}\left(\omega_{k}\right)\right]\left[1-P_{L^{\prime}, k+1}\left(\omega_{k+1}\right)\right]$. For a given $\omega_{k}$ and $\omega_{k+1}$, it will be optimal because no other rate allocations for $C_{L^{\prime}, k}\left(\omega_{k}\right)$ and $C_{L^{\prime}, k+1}\left(\omega_{k+1}\right)$ can give smaller values of $P_{L^{\prime}, k}\left(\omega_{k}\right)$ and $P_{L^{\prime}, k+1}\left(\omega_{k+1}\right)$. To optimize $C_{2 L^{\prime},(k+1) / 2}(\omega)$, then, it is sufficient to find $\omega_{k}$ and $\omega_{k+1}$, subject to $\omega_{k}+\omega_{k+1}=\omega$ to minimize the first-block-error probability. There are precisely $L^{\prime}-\left|L^{\prime}-\omega\right|+1$ different combinations of $\omega_{k}$ and $\omega_{k+1}$ that add to $\omega$. In particular, we have $\left(\omega_{k}, \omega_{k+1}\right) \in\{(0, \omega),(1, \omega-1), \ldots,(\omega, 0)\}$ for $0 \leq \omega \leq L^{\prime}$ and $\left(\omega_{k}, \omega_{k+1}\right) \in\left\{\left(\omega-L^{\prime}, L^{\prime}\right),\left(\omega-L^{\prime}+1, L^{\prime}-1\right), \ldots,\left(L^{\prime}, \omega-L^{\prime}\right)\right\}$ for $L^{\prime} \leq \omega \leq 2 L^{\prime}$. By examining all combinations, the optimal solution is

$$
\left(\omega_{k}^{*}, \omega_{k+1}^{*}\right)=\underset{\substack{\left(\omega_{k}, \omega_{k+1}\right) \\ \omega_{k}+\omega_{k+1}=\omega}}{\operatorname{argmin}}\left(1-\left[1-P_{L^{\prime}, k}\left(\omega_{k}\right)\right]\left[1-P_{L^{\prime}, k+1}\left(\omega_{k+1}\right)\right]\right)
$$

and the minimum first-block-error probability is

$$
P_{2 L^{\prime},(k+1) / 2}(\omega)=1-\left[1-P_{L^{\prime}, k}\left(\omega_{k}^{*}\right)\right]\left[1-P_{L^{\prime}, k+1}\left(\omega_{k+1}^{*}\right)\right] .
$$

The optimal rate allocation for $C_{2 L^{\prime},(k+1) / 2}(\omega)$ is

$$
\boldsymbol{\alpha}_{2 L^{\prime},(k+1) / 2}(\omega)=\left(\boldsymbol{\alpha}_{L^{\prime}, k}\left(\omega_{k}^{*}\right), \boldsymbol{\alpha}_{L^{\prime}, k+1}\left(\omega_{k+1}^{*}\right)\right)
$$

By repeating this procedure for all odd $k \in\left\{1, \ldots, N / L^{\prime}-1\right\}$ and $\omega \in\left\{0,1, \ldots, 2 L^{\prime}\right\}$, we can find the optimal rate allocation for all $N / 2 L^{\prime}$ outer codes of length $2 L^{\prime}$. Under the initial condition that $C_{L, k}(\omega)$ minimizes $P_{L, k}(\omega)$, it follows by induction that this algorithm will minimize $P_{L^{\prime}, k}(\omega)$ for all $L^{\prime} \in\left\{2^{l^{\prime}} \mid l^{\prime} \in\{l, \ldots, n\}\right\}$, leading to the optimal rate allocation for $C_{N, 1}(\omega)$ for all $\omega \in\{0,1, \ldots, N\}$. By selecting the solution for $\omega=K, \boldsymbol{\alpha}_{N, 1}(K)$ is the optimal rate allocation for the $(N, K)$ GCC-polar code. Although this algorithm itself is optimal, it requires exact knowledge of the first-block-error probabilities $P_{L, k}(\omega)$, whereas we only have the estimates from (6.3), which, in turn, are based on the inexact results from DE. As a result, our allocated rates are not strictly optimal. 


\subsubsection{Decoding Complexity}

The decoding complexity of the GCC-polar code mainly consists of two parts: SC decoding on the PC graph and ML decoding of the length- $L$ outer codes. In the decoding phase when the SC decoding is done, the decoding complexity is due to the computations of the LLRs according to (5.4) and (5.5). This decoding complexity is the same for both Arikan's code and the GCC-polar code. Let $\chi_{n, l}$ denote the complexity of this phase of decoding, i.e. corresponding to calculating the LLRs of all nodes at columns $l$ to $n$. The total decoding complexity for both Arikan's code and the GCC-polar code will be $\chi_{n, l}$ plus the complexity for decoding the outer codes of length $L=2^{l}$. Therefore, to compare the decoding complexity of the GCC-polar code and Arikan's it will suffice to find the complexity of decoding outer codes in the two cases.

We consider four types of operations to measure the decoding complexity. The addition of real numbers, the comparison of two real numbers, the product of two real numbers and the evaluation of the tanh (and $\tanh ^{-1}$ ) function ${ }^{1}$. For the Arikan outer code of dimension $\omega$, the SC decoder performs $\omega$ comparisons to find the sign of LLRs $\lambda_{i, 0}$. On the other hand, the ML decoding will not perform any tanh or product operations.

For an $(L, \omega)$ outer code, an exhaustive search ML decoder must calculate the metrics as in (8.2) for all the codewords in the codebook. However, lower complexity but still ML decoding can be performed by using the Viterbi algorithm on a suitably defined trellis representation of the linear block code [89]. To reduce the complexity of the Viterbi decoding of a linear block code, a minimum span generator matrix of the code is found for which a minimal trellis can be obtained. We use the Viterbi algorithm to decode the outer codes on their minimal trellises. In the Appendix we present the minimal trellises of the outer codes and their corresponding decoding complexities for $L=8$. The complexity of the Viterbi decoding of the outer codes is much less than that of the exhaustive search algorithm. For $\omega=L-1$, the generator matrix is in fact the even-parity code and thus the minimum of the absolute value of the LLRs is found and its hard decision value is flipped if the parity check equation is not satisfied (no additions and $2 L-1$ comparisons), and for $\omega=L$ no decoding is done as our outer code generator matrix is the identity matrix but $L$ comparisons are needed to find the sign of the LLRs. Table 6.1 lists the number of operations of each type

\footnotetext{
${ }^{1}$ A more comprehensive complexity analysis will highly depend on the software or hardware implementation and other factors such as the chip size, power consumption, CPU time, parallel computing and etc. However one can think that the number of actual computations of the four types can reflect the decoding complexity of the two methods to some extent. The proposed analysis is intended to be merely one valid and not a complete analysis.
} 
Table 6.1: The number of operations required for decoding Arikan's outer codes under SC decoding and the designed outer codes under ML decoding: The four types of operations are addition + , comparison $\leq$, product $\times$ and tanh evaluation.

\begin{tabular}{|c|c|c|c|c|c|c|c|c|}
\cline { 2 - 10 } & \multicolumn{4}{|c|}{ Arikan } & \multicolumn{4}{c|}{ GCC-polar } \\
\hline Outer code Rate & + & $\leq$ & $\times$ & $\tanh$ & + & $\leq$ & $\times$ & $\tanh$ \\
\hline \hline $0 / 8$ & & & & & & & & \\
\hline $1 / 8$ & 7 & 1 & 0 & 0 & 8 & 1 & 0 & 0 \\
\hline $2 / 8$ & 7 & 2 & 1 & 3 & 10 & 3 & 0 & 0 \\
\hline $3 / 8$ & 8 & 3 & 3 & 9 & 17 & 7 & 0 & 0 \\
\hline $4 / 8$ & 11 & 4 & 7 & 21 & 22 & 11 & 0 & 0 \\
\hline $5 / 8$ & 11 & 5 & 8 & 24 & 20 & 13 & 0 & 0 \\
\hline $6 / 8$ & 11 & 6 & 9 & 27 & 14 & 11 & 0 & 0 \\
\hline $7 / 8$ & 12 & 7 & 11 & 33 & 0 & 15 & 0 & 0 \\
\hline $8 / 8$ & 12 & 8 & 12 & 36 & 0 & 8 & 0 & 0 \\
\hline
\end{tabular}

for both Arıkan's outer codes and the designed ones listed in (6.5), for different rates with $L=8$.

As can be seen from this table, the number of operations for the two methods depends on the rate of the outer code. Therefore the overall complexity of the outer code decoding for the two methods depends on the fraction of the outer codes of each rate. Let $\Omega_{\omega}$ be the fraction of outer codes of dimension $\omega$ in a given GCC-polar code. That is $\Omega_{\omega}$ is the number of outer codes $C_{L, k}$ with $\omega_{k}=\omega$ divided by the total number of outer codes, $N / L$. This introduces a outer code rate distribution $\Omega(x)=\sum_{\omega=0}^{L} \Omega_{\omega} x^{\omega}$ for each of the two methods. For both Arikan's code and the GCC-polar code, once the outer code rate distributions are known, one can calculate the average number per outer code of each operation in Table I. Obviously these values will be zero for comparison operation for Arikan's code and the product and tanh operations for the GCC-polar code.

We compare the decoding complexities of some designed GCC-polar codes with Arikan's polar codes. We have designed three GCC-polar codes of rates $4 / 8,5 / 8$ and $6 / 8$ with $N=$ 1024 and outer code length $L=8$. The outer code rate distribution of these codes are shown in Table 6.2. Also shown is the outer code rate distributions of three conventional (Arikan) 
Table 6.2: Outer code rate distributions $\Omega_{\omega}$ for conventional and GCC-polar codes.

\begin{tabular}{|c|c|c|c|c|c|c|c|c|c|}
\hline \multirow{2}{*}{$\omega$} & 0 & 1 & 2 & 3 & 4 & 5 & 6 & 7 & 8 \\
\hline \hline Arikan-4/8 & .3125 & .1016 & .0156 & .0156 & .1172 & 0 & .0078 & .1328 & .2969 \\
\hline GCC-4/8 & .3281 & .0703 & .0313 & .0234 & .0781 & .0391 & .0156 & .1172 & .2969 \\
\hline Arikan-5/8 & .2344 & .0703 & .0078 & .0234 & .0781 & 0 & .0234 & .1094 & .4531 \\
\hline GCC-5/8 & .2344 & .0469 & .0234 & .0234 & .0625 & .0391 & .0156 & .1406 & .4141 \\
\hline Arikan-6/8 & .1328 & .0547 & .0234 & .0078 & .0625 & .0078 & .0156 & .0703 & .6250 \\
\hline GCC-6/8 & .1406 & .0391 & .0234 & .0234 & .0234 & .0391 & .0156 & .1016 & .5938 \\
\hline
\end{tabular}

Table 6.3: The average number of performed operations for outer code decodings of the codes in Fig. 6.5.

\begin{tabular}{|c|c|c|c|c|}
\hline Operation & + & $\leq$ & $\times$ & $\tanh$ \\
\hline \hline Arikan-4/8 & 7.4 & 4.0 & 5.9 & 17.9 \\
\hline GCC-4/8 & 3.9 & 6.0 & 0.0 & 0.0 \\
\hline Arikan-5/8 & 8.6 & 4.9 & 7.4 & 22.4 \\
\hline GCC-5/8 & 3.38 & 7.07 & 0.0 & 0.0 \\
\hline Arikan-6/8 & 9.8 & 5.99 & 8.9 & 26.8 \\
\hline GCC-6/8 & 2.45 & 7.48 & 0.0 & 0.0 \\
\hline
\end{tabular}

polar codes with the same overall rates and code lengths. Table 6.3 shows the average number of operation required for decoding the outer codes of these six codes. As can be seen, Arikan's codes require a smaller number of comparisons and additions while the GCC-polar codes require no product or tanh operations. Although the relative complexity of the different types of operations depend heavily on their hardware implementations, additions and comparisons tend to be easier to implement than product and tanh. Even if all operations are assumed to be equally difficult, Table 6.3 shows that resultant GCC-polar codes have smaller decoding complexities than Arikan's. In Section IV we compare the performance of the GCC-polar codes with Arikan's. 


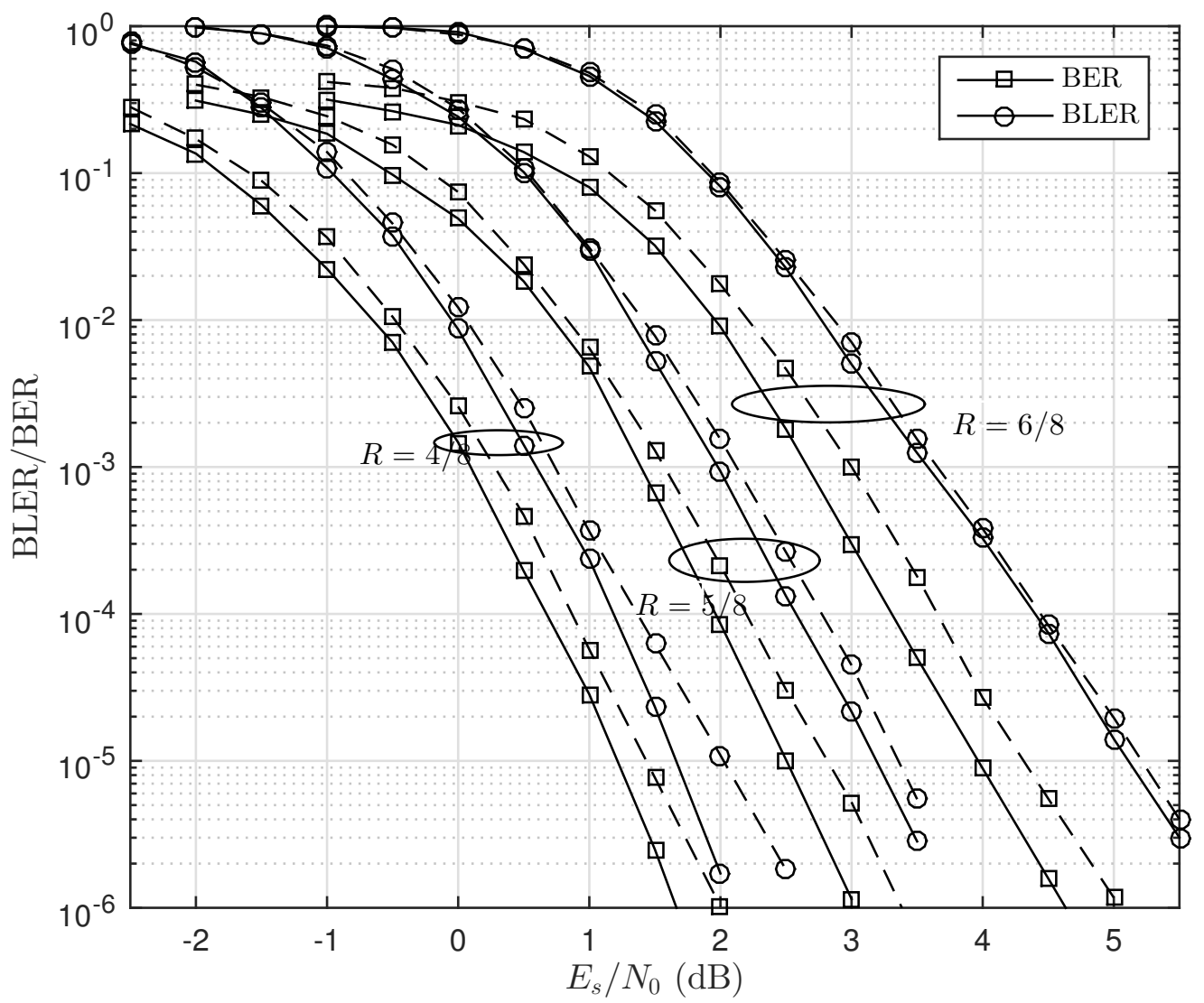

Figure 6.4: The performance comparison of the GCC-polar code (solid lines) and the conventional polar code (dashed lines) for different code rates, with $N=256$ and $L=8$.

\subsection{Performance Evaluation Results}

In this section we present Monte-Carlo simulation results to evaluate the performance of the GCC-polar codes. We assume the underlying channel $\mathcal{W}$ is a BI-AWGN channel with BPSK with mapping $0 \rightarrow+1$ and $1 \rightarrow-1$. We have designed GCC-polar codes for different rates with the bank of outer codes of length $L=8$ given in (6.5). Fig. 6.4 depicts BLER and BER for codes of different rates for $N=256$. The rate allocation algorithm proposed in Section III is used to find the optimal rates for the outer codes. For comparison, the performance of Arikan's polar code, designed by the DE method proposed in [73], are also shown. As can be seen, due to the superior performance of the outer codes over Arikan's and the appropriate rate allocation, the GCC-polar codes outperform 
Arikan's in terms of both BLER and BER. It can also be seen that the coding gain in terms of the BER is larger than that of the BLER. This seems to be due to the fact that for both Arikan and the GCC-polar code, there are a significant number of outer codes with a rate of 1, mainly in the very low part of the PC graph as well as the graph of the GCC code in Fig. 6.2. Arikan's codes use the generator matrix $\mathbf{G}_{8}$ for these outer codes while our scheme uses the identity matrix $\mathbf{I}_{8}$. From theoretical analysis it can be proved that among all rate- 1 codes for transmission over a BIMOS channel, the one with an identity matrix as the generator matrix has the smallest BER (i.e., an uncoded system would minimize the BER if the rate of the code is 1). Heuristically, it is because a rate- 1 code with identity generator matrix does not add any interference between the information bits, while in other cases the code bits are summations of the information bits and thus the information bits play as interference to each other, resulting in a higher bit error propagation. Now for the number of outer codes of rate $1\left(\omega_{k}=L\right)$, the decoder of the GCC-polar code gets to a point where it has to decode a rate-1 outer code. Although for both Arikan's and our outer code the resultant BLER is the same, the identity matrix outer code outperforms Arikan's in terms of BER. This means that we have more outer codes to participate in improving the BER than the BLER. This seems to be the reason why the coding gain of the GCC-polar code over Arikan's code in terms of the BER is larger than in terms of BLER.

We have also designed GCC-polar codes for $N=1024$. Fig. 6.5 shows the BLER and BER results for the codes designed at this length. As can be seen, the coding gain is still realized at the larger length $N=1024$. At any code length $N$ the amount of coding gain varies from rate to rate. This seems to be due to different resultant outer code rate distributions. The rate distribution $\Omega(x)$ not only plays an important role in the performance of both codes, but also determines the overall decoding complexity for the outer codes. Ideally, for the GCC-polar code we would like all the outer codes to be either of rates 0 or 1 as these rates will imply zero decoding complexity as in Table 6.1. However this choice of the rate distribution may not necessarily result in good GCC-polar codes. The proposed algorithm allocates the rates to the outer codes with the sole goal of improving the performance of the GCC-polar code without any constraints to meet a certain level of decoding complexity. However as Table 6.2 and 6.3 show the resultant decoding complexity of the GCC-polar codes in Fig. 6.5 is even reduced compared to the conventional polar codes.

We also compared the GCC-polar codes designed with the proposed rate allocation algorithm to GCC-polar codes constructed from the equal error probability rule rate allocation algorithm proposed in [86]. Both algorithms are used to allocate the rates to the outer 


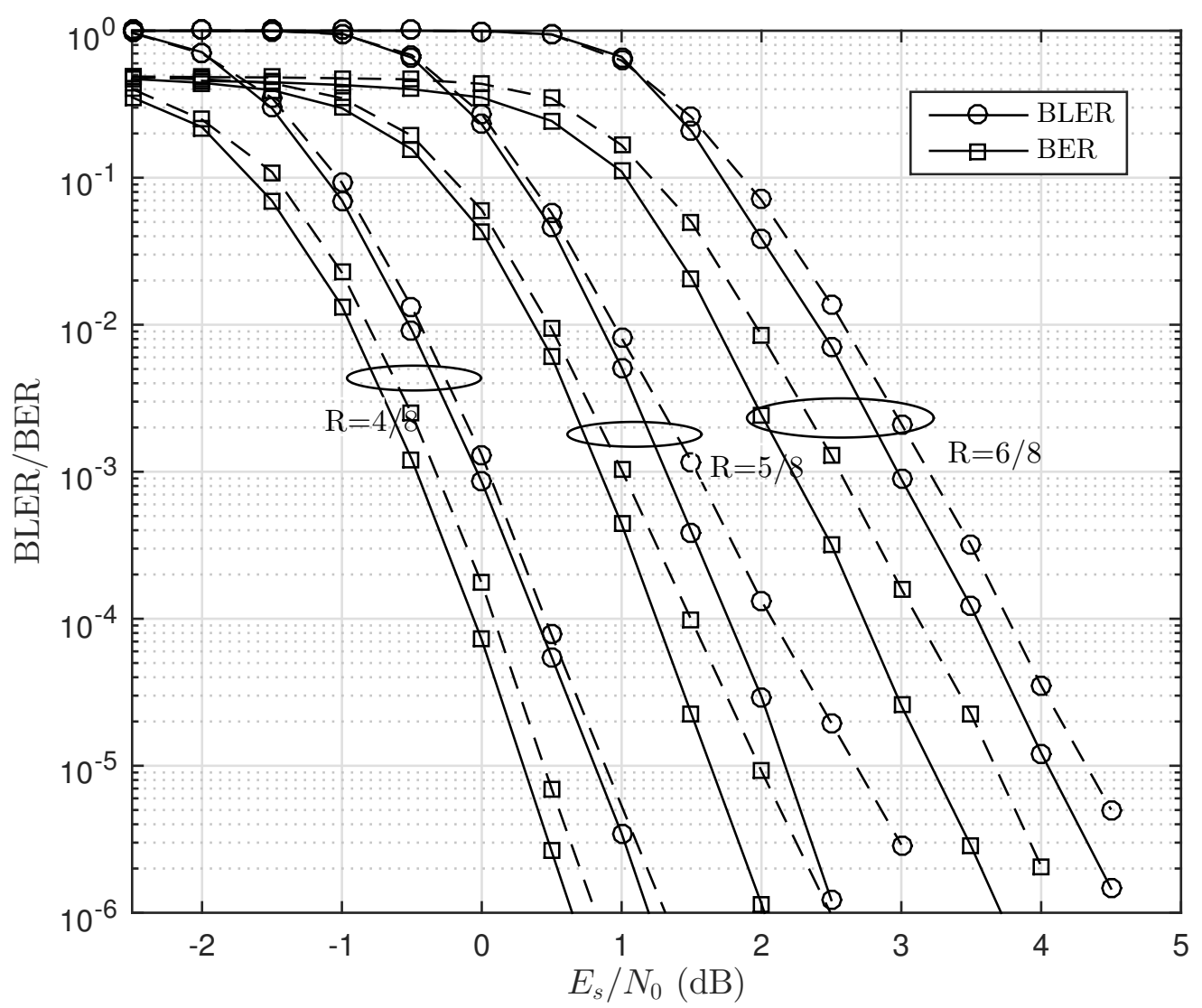

Figure 6.5: The performance comparison of the GCC-polar code (solid lines) and the conventional polar code (dashed lines) for different code rates, with $N=1024$ and $L=8$.

codes of a $N=1024$ GCC-polar code with the bank of outer codes of length $L=8$ given in (6.5). As can be seen from Fig. 6.6 the proposed rate allocation algorithm outperforms the Equal error probability rule for different rates. It should be also noted that these two rate allocation algorithms have also resulted in different decoding complexities. The rate distributions and the average number of performed decoding operations per outer code for the GCC-polar codes constructed from the equal error probability rule, are shown in Tables 6.4 and 6.5 respectively. It can be seen that the average number of decoding operations per outer code for GCC-polar codes of rates 4/8, 5/8 and 6/8 are 10.22, 10.72 and 10.08 respectively while these quotients for the codes constructed from the proposed rate allocation algorithms are 9.99, 10.45 and 9.94 respectively (see Table 6.3). Therefore the proposed 


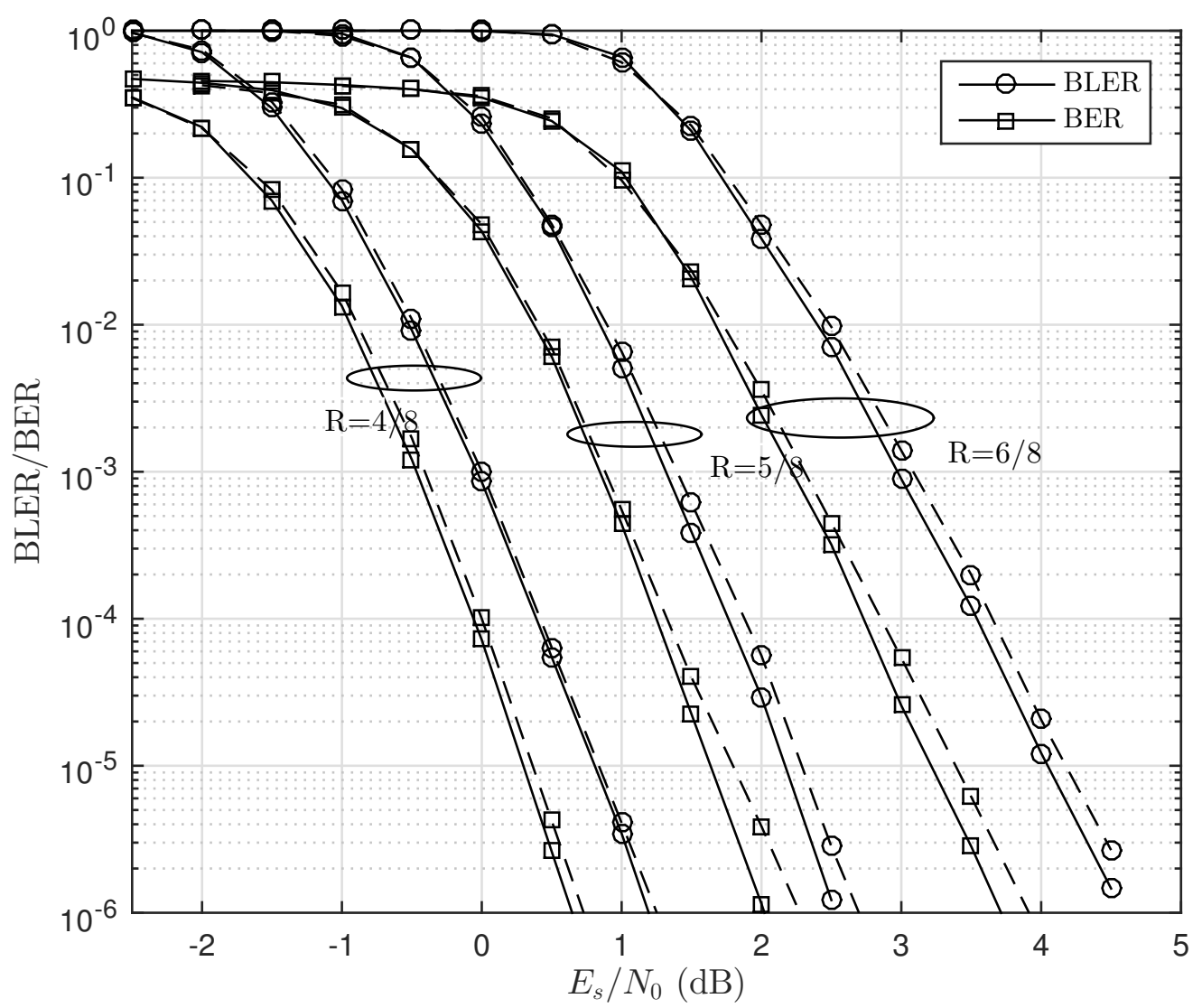

Figure 6.6: The impact of the rate allocation algorithm on the performance of GCC-polar codes. Solid lines correspond to the proposed rate allocation algorithm while the dashed lines correspond to the equal error probability rule.

rate allocation algorithm has also reduced the decoding complexity of the outer codes. It is noteworthy that since the proposed rate allocation algorithm considers the BLER of the GCC-polar code, it is also possible to design the GCC-polar codes for a target BLER and aim for minimizing the decoding complexity of outer codes. Such an approach is not possible for the equal error probability rule rate allocation algorithm.

Since the best performance of Arikan's code are achieved under the CRC-aided successive cancellation list (CA-SCL) decoding [63], it will be useful to develop a list decoder for the proposed GCC-polar codes. To this end, we modify the traditional list decoder to adapt to the outer code structure of the GCC-polar codes. Unlike the traditional SCL decoder 
Table 6.4: Outer code rate distributions $\Omega_{\omega}$ for GCC-polar codes using the rate allocation algorithm in [86].

\begin{tabular}{|c|c|c|c|c|c|c|c|c|c|}
\hline \multicolumn{1}{|c|}{$\omega$} & 0 & 1 & 2 & 3 & 4 & 5 & 6 & 7 & 8 \\
\hline \hline $4 / 8$ & .3203 & .0781 & .0313 & .0234 & .0781 & .0391 & .0313 & .1016 & .2969 \\
\hline $5 / 8$ & .2188 & .0547 & .0313 & .0391 & .0469 & .0391 & .0391 & .1172 & .4141 \\
\hline $6 / 8$ & .1250 & .0469 & .0391 & .0234 & .0234 & .0313 & .0391 & .0625 & .6094 \\
\hline
\end{tabular}

Table 6.5: The average number of performed operations for outer code decodings of the codes in Table 6.4 .

\begin{tabular}{|c|c|c|c|c|}
\hline Rate Operation & + & $\leq$ & $\times$ & $\tanh$ \\
\hline \hline $4 / 8$ & 4.27 & 5.94 & 0.0 & 0.0 \\
\hline $5 / 8$ & 3.77 & 6.94 & 0.0 & 0.0 \\
\hline $6 / 8$ & 2.85 & 7.23 & 0.0 & 0.0 \\
\hline
\end{tabular}

where the list is expanded and pruned at each time index, the list decoder for the GCCpolar code, expands and prunes the list at time indices $\omega_{1}, \omega_{2}, \ldots, \omega_{i}, \ldots$. Recall that $\omega_{i}$ is the dimension of the $i$-th outer code. With a list size of $\rho$, at time $\omega_{i}$, the list consists of $\rho$ message sequences $u_{1}^{k_{i}}$, where $k_{i}=\sum_{j=1}^{i} \omega_{i}$. Each list member is assigned a metric reflecting a posteriori probability of the member. At time $\omega_{i+1}$ the list is expanded by appending all $2^{\omega_{i+1}}$ possible $\omega_{i+1}$ bits to each member. The metric for each extended member is calculated and then the same procedure of sorting the metrics and pruning the list by keeping the $\rho$ members with the largest metrics is taken. Define the metric of a list member $u_{1}^{k_{i}}$ as $M\left(u_{1}^{k_{i}}\right)=\log \left(\operatorname{Pr}\left(u_{1}^{k_{i}} \mid y_{1}^{N}\right)\right)$, calculating the metrics of a member $u_{1}^{k_{i}}$ can be done as follows.

$$
M\left(u_{1}^{k_{i}}\right)=M\left(u_{1}^{k_{i-1}}\right)-\sum_{j=1}^{L} c_{j} \lambda_{j}-\log \sum_{\mathbf{c} \in C_{L, i}} e^{-\sum_{j=1}^{L} c_{j} \lambda_{j}}
$$

where $\left[\lambda_{1}, \ldots, \lambda_{L}\right]$ are the LLRs calculated by the SC decoder of the GCC-polar code at the output nodes of $C_{L, i}$ when $u_{1}^{k_{i-1}}$ are known to the decoder.

Fig. 6.7 shows the BLERs of the rate-half GCC-polar code under the proposed SCL de- 


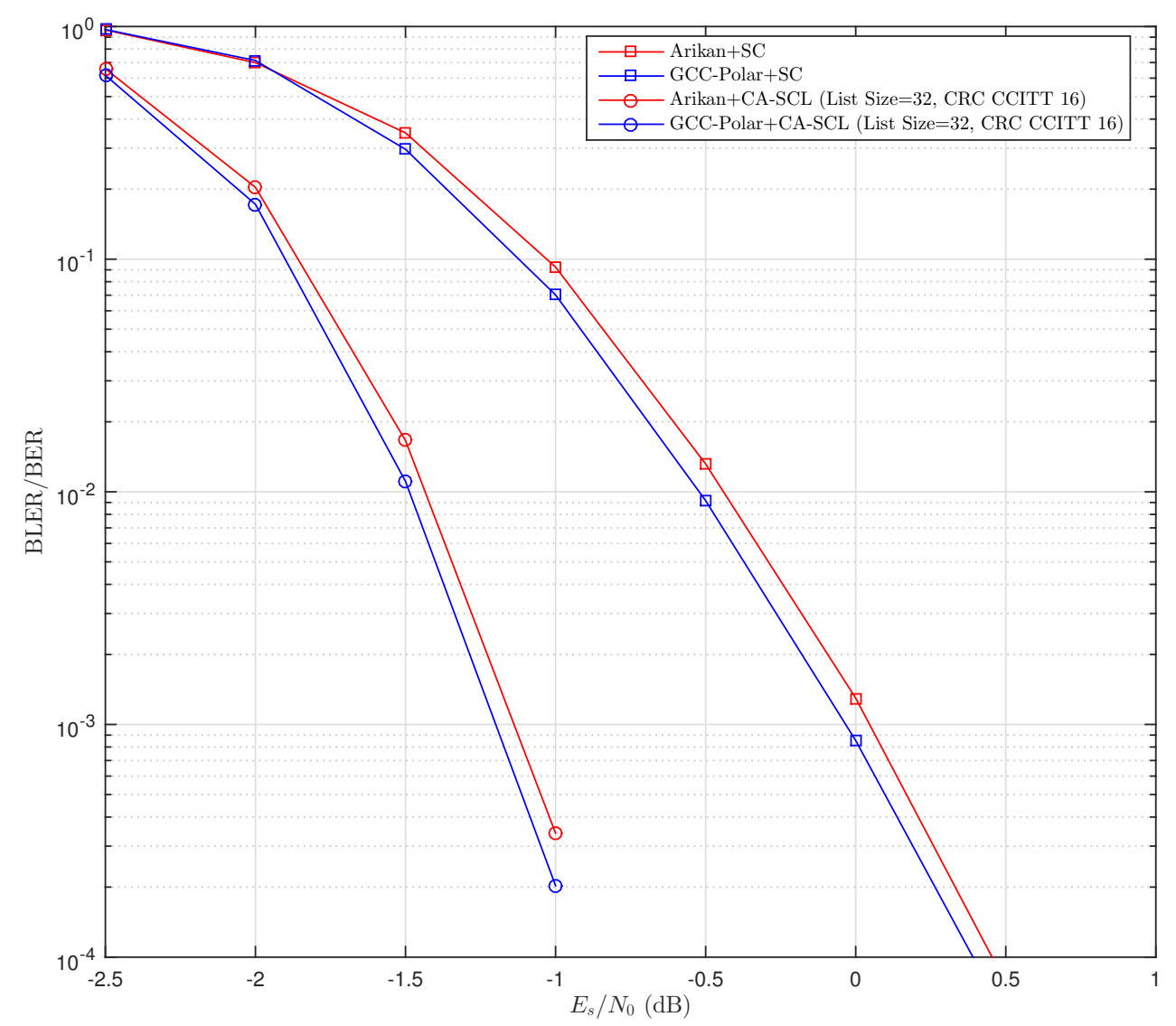

Figure 6.7: Block error rates of Arikan and GCC-polar codes under SC and CA-SCL decoding with a list size of 32 .

coding with CRC. The well-known 16-bit CRC-16-CCITT is used. As can be seen the same amount of gain that was realized under SC decoding is accomplished under CA-SCL decoding. The gain appears to be a bit larger at higher SNRs. Note that the difference in the complexity of the GCC-polar code under the proposed SCL decoder and Arikan's code is mainly due to calculating (6.10). For each member in the list the decoder calculates the LLRs at the output nodes of the current outer code. The list member is extended by the number of bits equal to the dimension of the current outer code (in case of Arikan each member is extended by $1 \mathrm{bit}$ ). Then the codebook of the outer code, $C_{L, i}$, is used to calculate the incremental term in (6.10) and in turn the metric of the extended member. We stress again that these improvements over the Arikan code under SC decoding, is brought by a significant increase in decoding complexity due to the list operations, while the GCC-polar 
codes achieve improvement while reducing the complexity. However this coding gain is far less than that of Arikan or GCC-polar code under list decoding.

Although performing computer simulations at very large code lengths may not be easy, it might be possible to anticipate the behaviour of the GCC-polar codes at very large $N$. For any fixed value of outer code length $L$, as $N$ tends to infinity, the channels seen by the outer codes begin to polarize. This is because the part of the graph to the right of the outer codes in Fig. 6.2 consists of polar codes of length $N / L$ which as $N / L$ gets larger they introduce more polarization. As a result the LLRs at the output of the outer code $C_{L, k}$, i.e. $\lambda_{i, l} i \in \mathcal{S}_{k}$, are either very good or very bad. A good rate allocation algorithm would result in outer code rates in such a way that almost all the outer codes are given a rate of either zero or one. As seen in Table 6.1 this implies zero ML decoding complexity for these outer codes. Moreover the optimal outer codes of rate 0 and 1 are simple to find (for rate 0 it always generates the all-zero codeword and for rate 1 it is the identity matrix of size $L$ ). So the GCC-polar code will have smaller decoding complexity than Arikan's. Since Arikan's code approaches capacity for large $N$, the GCC-polar code cannot substantially outperform it in term of the BLER. The same can be said for the BER. However due to the BER-improving rate-1 outer codes, it is expected that the gain in terms of BER remains longer. Preliminary results for $N=65536$ support this hypothesis. For very large code lengths no substantial coding gain over Arikan's code is expected for BLER or BER.

For fixed length $N$ of the GCC-polar code, we also anticipate that increasing $L$ brings coding gain over Arikan's code. If $L=1$ the GCC-polar codes will reduce to Arikan's codes and the rate allocation process will be the counterpart of determining the information and frozen sets. As $L$ increases, it is possible to find better outer codes than Arikan's (under ML decoding) and thus (as we have seen for $L=8$ ) the GCC-polar code will have larger coding gain over Arikan's. Hypothetically, if we could increase the outer code length to $L=N$ and use ML decoding for the outer codes a significant coding gain could be expected but this is obviously infeasible for large values of $L$ as it will cause impractical complexity both for designing the outer codes and decoding them with an ML decoder. Therefore, except for fairly small outer code lengths (such as $L=8$ ), this prevents the GCC-polar codes from being used in practical systems. In this case it is possible to employ suboptimum codes with suboptimum decoders, such as short LDPC codes, in place of the outer codes $C_{L, k}$. This will allow the advantages of larger $L$ to be realized, while still keeping the complexity at a reasonable level (although likely more complex than conventional polar codes).

As an alternative to using suboptimum codes and suboptimum decoders for the outer codes 
for large $L$, a different approach can be taken. We note that for large $N$, all but a vanishing fraction of the outer codes are either of rates zero or one. The optimum outer codes at these rates are both simple to design and decode. Outer codes of rate $\frac{L-1}{L}$ are also easy to decode because they are even-parity codes which only require $2 L-1$ comparisons for ML decoding. As a result one can combine adjacent length- $L$ outer codes of rate 1 into a larger outer code of the same rate. A similar action can be performed to use larger even-parity outer codes. This will allow for using different outer code lengths in different parts of the graph of the GCC-polar code without any increase in decoding complexity. Although this will not have any effect on the BLER it is expected to improve the BER of the GCC-polar code due to the large rate- 1 identity matrix outer codes.

\subsection{Conclusion}

We have presented a framework for designing GCC-polar codes with very short outer code lengths $(L \leq 8)$. At these outer code lengths it is possible to employ density evolution to design the outer codes for the actual channels seen by them with the goal of minimizing their BLER. We proposed a rate-allocation algorithm to determine the rates of the outer codes of the GCC-polar code. It was shown that the proposed GCC-polar code construction method can bring about $0.5 \mathrm{~dB}$ coding gain over the conventional polar codes. We have also seen that the proposed rate allocation algorithm can result in superior GCC-polar codes with less decoding complexity compared to the previous rate allocation algorithm. This decrease in decoding complexity is due to the resultant rate distributions for the outer codes. Although it is not guaranteed that the proposed algorithm will always result in codes with lower complexity, it appears to be the case for our constructed codes. The GCC-polar codes can also be used for arbitrary outer codes of different lengths and different classes provided that a low complexity decoder is available for their decoding. This open the possibility of using well-known class of error control codes such as LDPC codes as the outer codes and produce a combined LDPC-polar coding scheme. 


\section{Chapter 7}

\section{Conclusion and Future Works}

In this thesis we studied the design of channel codes for use in IR-HARQ schemes. We considered RB-HARQ and aimed to reduce the overhead of the feedback channel to allow the scheme to be used in practical communication systems. The proposed scheme measures the reliability of clusters of adjacent code bits rather than individual bits. Several reliability metrics are derived analytically. Our simulation results show that an efficient reliability metric can result in up to $2 \mathrm{~dB}$ gain over the previous RB-HARQ schemes. After that, we proposed a new framework of code design by designing a sequence of RC codes whose nature changes from LDPC to LDGM codes as their rate decreases. The proposed RC codes, referred to as RC-LDPC/LDGM codes, can be used for IR-HARQ schemes that benefit from the advantages of both IR-HARQ schemes based on LDPC and LDGM codes. We have presented a combined decoding of the RC-LDPC/LDGM codes on both their generator and parity check graphs and shown it can result in significant coding gain compared to decodings on individual graphs. At the expense of an increased decoding complexity and a small performance loss at the intermediate SNRs, the proposed method results in throughput that remains within $1 \mathrm{~dB}$ of the Shannon capacity for a wide range of SNR. Then we considered polar codes and introduced the concept of polar code extending as an effective method to construct polar codes for IR-HARQ schemes. The extending method allows a possibly repeated transmission of code bits from a set of code bits of size $N_{M} \log _{2} N_{M}+K$, for a mother polar code of length $N_{M}$ with message size $K$. We presented a throughput-specific extending algorithm to extend a possibly punctured polar code. This method, in conjunction with the proposed puncturing method results in a universal capacity-approaching IRHARQ scheme that can be implemented with the same encoding and decoding complexity as the original polar mother code. Finally we studied the design of an improved type of polar coding scheme referred to as GCC-polar codes. The idea of the GCC-polar code is 
to replace the small outer codes of the Arikan's code, with arbitrary stronger codes. We employed density evolution to design the outer codes for the actual channels seen by them with the goal of minimizing their BLER. For a given set of outer codes, a rate-allocation algorithm is proposed to determine the rates of the outer codes of the GCC-polar code. It is shown that the proposed GCC-polar code construction method can bring about $0.5 \mathrm{~dB}$ coding gain over the conventional polar codes. We have also seen that the proposed rate allocation algorithm can result in superior GCC-polar codes with less decoding complexity compared to the previous rate allocation algorithm.

We briefly presents some possible future works for this thesis. With the proposed IR-HARQ scheme based on polar codes, we note that finding the best next $N_{E}$ extending nodes from the set of code bits of size $N_{M} \log _{2} N_{M}+K$ can be computationally infeasible for large values of $N_{M}$ as the size of the search space is $\left(N_{M} \log _{2} N_{M}+K\right)^{N_{E}}$ which grows exponentially with $N_{E}$. The proposed method is one search method to find these extending nodes by selecting them one at a time from within a certain subset of all nodes in the PC graph. One drawback of the proposed method is that it is greedy. As an attempt to reduce the greediness, finding the best $D$ extending nodes at a time can be considered in conjunction with an efficient search algorithm. Also one could think of other search methods which can search for the best extending node from within the entire graph, as opposed to from within a subset of the graph, while maintaining an acceptable search complexity.

Although the proposed method based on the combined LDPC and LDGM codes, can decrease the gap to the channel capacity significantly, it comes with an increase in the decoding complexity mainly due to the high weight columns of the extended generator matrix for the LDPC mother code. The number of such highly complex columns is proportional to the rate of the LDPC mother code. The higher the rate of the mother code, the lower the number of such complex columns. However as we saw increasing the rate of the LDPC mother code comes with a performance loss at high SNRs. To decrease the decoding complexity we have to decrease the weight of extended columns. Since the choice of the systematic part of the extended generator matrix is arbitrary, and the non-systematic part is a function of the systematic part and the parity check matrix of the LDPC code, perhaps a joint approach to construct the LDPC and LDGM code can be considered. With such an approach, we will look for parity check matrices for which it is possible to find an "optimized" pair of $\mathbf{G}_{s}$ and $\mathbf{G}_{p}$ in a sense that increases the sparsity of the generator matrix.

Another possible future work can be proposed on improving the method of extending the parity check matrix of the LDPC mother code. Consider a given LDPC mother code with 
certain degree distribution and suppose it is extended by some extending matrices according to the structure in Chapter 3. The degree distribution of the extended parity check matrix can be obtained from the degree distribution of the mother code and that of the extending matrix using the convolution of the two degree distributions. One could consider an asymptotic approach to extend the parity check matrices by optimizing a sequence of extending degree distributions such that the threshold of the BP decoder for each extended code is minimized. 


\section{Chapter 8}

\section{Appendix}

\subsubsection{Linear Block Codes over BIMOS Channels: An ML Decoder and an Upper Bound on their BLER}

Consider an $(N, K)$ linear block code for transmission over a BIMOS channel with output $y$ whose transition probabilities are $f_{0}(y)=f_{Y}(y \mid 0)$ and $f_{1}(y)=f_{Y}(y \mid 1)$, and consider an

equivalent channel constructed from this channel with output $\lambda=\log \frac{f_{0}(y)}{f_{1}(y)}$, with transition probabilities $p_{0}(\lambda)=f_{\lambda}(\lambda \mid 0)$ and $p_{1}(\lambda)=f_{\lambda}(\lambda \mid 1)$. Since the channel is BIMOS, the LLRs of the input bit given the received value $y_{i}$ (i.e. $\lambda_{i}$ ) are sufficient statistics for ML decoding, so these two channels are equivalent. Moreover it implies that $p_{0}(\lambda)=p_{1}(-\lambda)$. Let $\mathbf{y}=$ $\left[y_{1}, \ldots, y_{N}\right]$, or $\lambda=\left[\lambda_{1}, \ldots, \lambda_{N}\right]$ be the received vectors, where $\lambda_{i}=\log \frac{f_{Y}\left(y_{i} \mid 0\right)}{f_{Y}\left(y_{i} \mid 1\right)}$. The ML decoder chooses the decoded codeword $\hat{\mathbf{c}}$ as

$$
\begin{aligned}
\hat{\mathbf{c}} & =\operatorname{argmax}_{\mathbf{c}=\left[c_{1}, \ldots, c_{N}\right]} \prod_{i=1}^{N} f_{Y}\left(y_{i} \mid c_{i}\right)=\operatorname{argmax}_{\mathbf{c}=\left[c_{1}, \ldots, c_{N}\right]} \prod_{i=1}^{N} \frac{f_{Y}\left(y_{i} \mid c_{i}\right)}{f_{Y}\left(y_{i} \mid 0\right)} \\
& =\operatorname{argmax}_{\mathbf{c}=\left[c_{1}, \ldots, c_{N}\right]} \prod_{i \in D(\mathbf{c})} \frac{f_{Y}\left(y_{i} \mid c_{i}\right)}{f_{Y}\left(y_{i} \mid 0\right)} \\
& =\operatorname{argmax}_{\mathbf{c}=\left[c_{1}, \ldots, c_{N}\right]} \sum_{i \in D(\mathbf{c})} \log \frac{f_{Y}\left(y_{i} \mid c_{i}\right)}{f_{Y}\left(y_{i} \mid 0\right)} \\
& =\operatorname{argmax}_{\mathbf{c}=\left[c_{1}, \ldots, c_{N}\right]} \sum_{i \in D(\mathbf{c})}-\lambda_{i},
\end{aligned}
$$


where, for a codeword $\mathbf{c}=\left[c_{1}, \ldots, c_{N}\right], D(\mathbf{c})$ denotes the set of indices $i$ for which $c_{i}=1$. Since $c_{i}=1$ for all $i \in D(\mathbf{c}),(8.1)$ can be written as

$$
\begin{aligned}
\hat{\mathbf{c}} & =\operatorname{argmax}_{\mathbf{c}=\left[c_{1}, \ldots, c_{N}\right]} \sum_{i \in D(\mathbf{c})}-c_{i} \lambda_{i}=\operatorname{argmax}_{\mathbf{c}=\left[c_{1}, \ldots, c_{N}\right]} \sum_{i=1}^{N}-c_{i} \lambda_{i} \\
& =\operatorname{argmin}_{\mathbf{c}=\left[c_{1}, \ldots, c_{N}\right]} \mathcal{L}(\mathbf{c}),
\end{aligned}
$$

where $\mathcal{L}(\mathbf{c})=\sum_{i=1}^{N} c_{i} \lambda_{i}$ is defined as the metric of codeword c. Eq. (8.2) is the decoding rule for block-wise ML decoding in terms of the LLRs of the code bits ${ }^{1}$.

Assuming that the all-zero codeword $\mathbf{0}$ is transmitted over the channel, the decoding error probability, $P_{\mathcal{E}}$, can be upper bounded by the union bound as

$$
\begin{aligned}
P_{\mathcal{E}} & \leq \sum_{\mathbf{c} \neq \mathbf{0}} \operatorname{Pr}(\mathcal{L}(\mathbf{c}) \leq \mathcal{L}(\mathbf{0}) \mid \mathbf{0} \text { transmitted }) \\
& =\sum_{\mathbf{c} \neq \mathbf{0}} \operatorname{Pr}(\mathcal{L}(\mathbf{c}) \leq 0 \mid \mathbf{0} \text { transmitted }) .
\end{aligned}
$$

Since

$$
\begin{aligned}
& \operatorname{Pr}(\mathcal{L}(\mathbf{c}) \leq 0 \mid \mathbf{0} \text { transmitted }) \\
& =\operatorname{Pr}\left(\sum_{i=1}^{N} c_{i} \lambda_{i} \leq 0 \mid \mathbf{0} \text { transmitted }\right) \\
& =\operatorname{Pr}\left(\sum_{i \in D(\mathbf{c})} \lambda_{i} \leq 0 \mid \mathbf{0} \text { transmitted }\right)
\end{aligned}
$$

by defining $Z=\sum_{i \in D(\mathbf{c})} \lambda_{i}$, we have

$$
\operatorname{Pr}(\mathcal{L}(\mathbf{c}) \leq 0 \mid \mathbf{0} \text { transmitted })=\int_{-\infty}^{0} f_{Z}(z \mid \mathbf{0} \text { transmitted }) d z
$$

Given that the all-zero codeword is transmitted, $Z$ is the summation of independent random

\footnotetext{
${ }^{1}$ In general, it is also possible to perform the ML decoding of any linear block code more efficiently by first finding a minimal trellis of the code, and then using the Viterbi algorithm. The advantage of the Viterbi decoding is more noticeable for larger outer codes. Since we would like to decrease the overall decoding complexity of Arikan's code, outer codes with low state (or branch) complexity should be found (the state/branch complexity of the trellis of linear codes can grow exponentially with the code length). Unless one designs the outer codes in such a way that a low state complexity can be provided, the overall complexity of the GCC-polar codes will be most likely larger than that of Arikan's code.
} 
variables $\lambda_{i}$, so the density of $Z$ is the convolution of densities of $\lambda_{i}, p_{0}(z)$. Thus

$$
f_{Z}(z \mid \mathbf{0} \text { transmitted })=p_{0}^{\otimes d}(z)
$$

where $d$ is the Hamming weight of $\mathbf{c}$, and $p_{0}^{\otimes d}(z)$ denotes the convolution of $p_{0}(z)$ with itself $d$ times. So

$$
\operatorname{Pr}(\mathcal{L}(\mathbf{c}) \leq 0 \mid \mathbf{0} \text { transmitted })=\int_{-\infty}^{0} p_{0}^{\otimes d}(z) d z
$$

Let $\gamma_{d}=\int_{-\infty}^{0} p_{0}^{\otimes d}(z) d z$. If there are $A_{d}$ codewords with a Hamming weight of $d$, it follows from (8.3) and (8.8) that

$$
P_{\mathcal{E}} \leq \sum_{d} A_{d} \gamma_{d}
$$

Since the BLER is independent of the transmitted codeword the above bound is in fact the actual upper bound on the BLER under ML decoding.

\subsubsection{Minimal Trellises of the Designed Outer Codes of Length $L=8$}

The outer codes of the GCC-polar codes are decoded using the Viterbi algorithm on the minimal trellises of the codes. Here we present minimal trellises and their equivalent minimum span generator matrices (MSGMs). For a trellis with $|E|$ edges and $|V|$ nodes, the Viterbi algorithm requires $\frac{|E|}{2}$ additions and $|E|-|V|-1$ comparisons. The MSGM, $\mathbf{G}_{k}$, of the outer code of rate $k / 8$ for $k=1, \ldots, 6$ is as follows.

$$
\begin{aligned}
\mathbf{G}_{1} & =\left[\begin{array}{llllllll}
1 & 1 & 1 & 1 & 1 & 1 & 1 & 1
\end{array}\right] . \\
\mathbf{G}_{2} & =\left[\begin{array}{llllllll}
1 & 1 & 1 & 1 & 1 & 0 & 0 & 0 \\
0 & 0 & 0 & 1 & 1 & 1 & 1 & 1
\end{array}\right] .
\end{aligned}
$$




$$
\begin{aligned}
\mathbf{G}_{3} & =\left[\begin{array}{llllllll}
1 & 1 & 0 & 1 & 1 & 0 & 0 & 0 \\
0 & 1 & 1 & 1 & 0 & 1 & 0 & 0 \\
0 & 0 & 0 & 1 & 1 & 1 & 1 & 1
\end{array}\right] . \\
\mathbf{G}_{4} & =\left[\begin{array}{llllllll}
1 & 1 & 1 & 1 & 0 & 0 & 0 & 0 \\
0 & 1 & 1 & 0 & 1 & 1 & 0 & 0 \\
0 & 0 & 1 & 1 & 0 & 1 & 1 & 0 \\
0 & 0 & 0 & 0 & 1 & 1 & 1 & 1
\end{array}\right] . \\
\mathbf{G}_{5} & =\left[\begin{array}{llllllll}
1 & 1 & 1 & 1 & 0 & 0 & 0 & 0 \\
0 & 1 & 0 & 1 & 0 & 1 & 0 & 0 \\
0 & 0 & 1 & 1 & 1 & 0 & 0 & 0 \\
0 & 0 & 0 & 0 & 1 & 1 & 1 & 0 \\
0 & 0 & 0 & 0 & 0 & 0 & 1 & 1
\end{array}\right] .
\end{aligned}
$$

The corresponding minimal trellises are shown in Figures 8.1 to 8.6. 


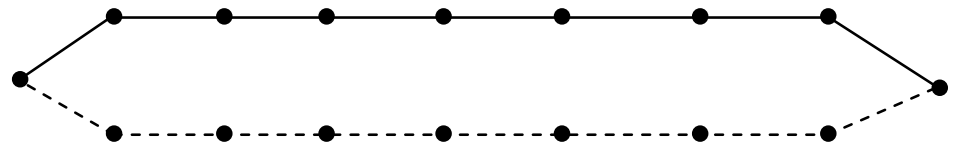

Figure 8.1: The minimal trellis of $\mathbf{G}_{1}$.

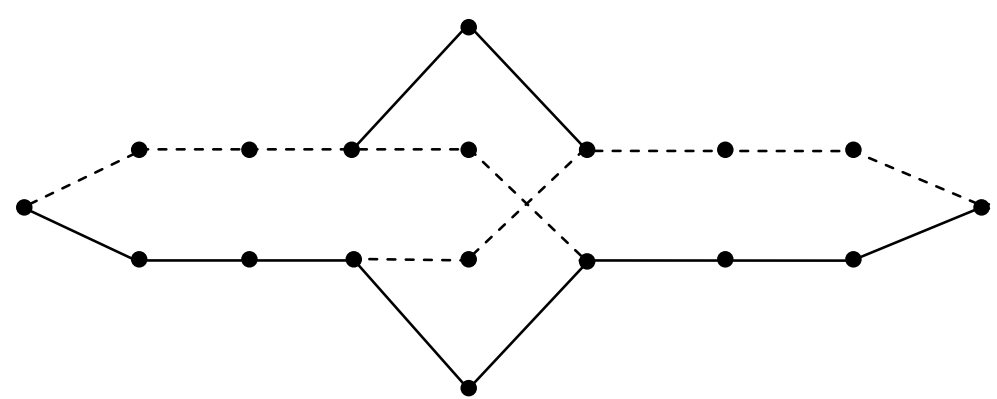

Figure 8.2: The minimal trellis of $\mathbf{G}_{2}$.

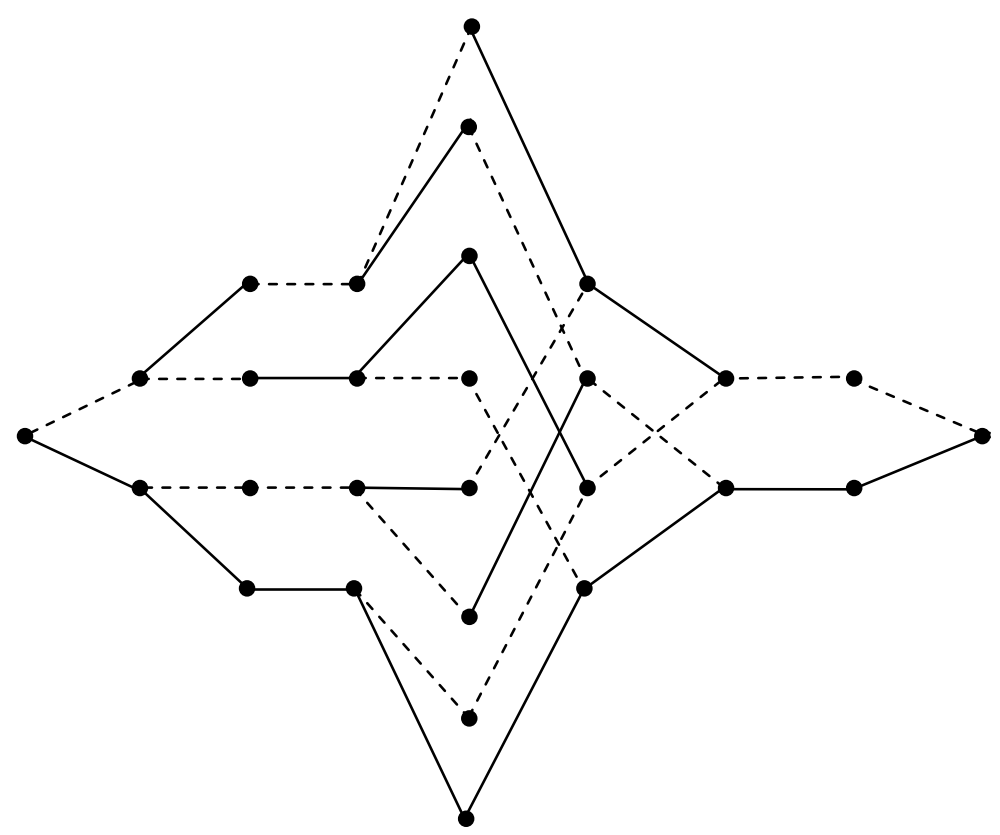

Figure 8.3: The minimal trellis of $\mathbf{G}_{3}$

The Viterbi decoding complexities of these codes can be obtained by calculating the number of edges and vertices of the trellis diagram. Recall that for the rates $7 / 8$ the ML decoder 


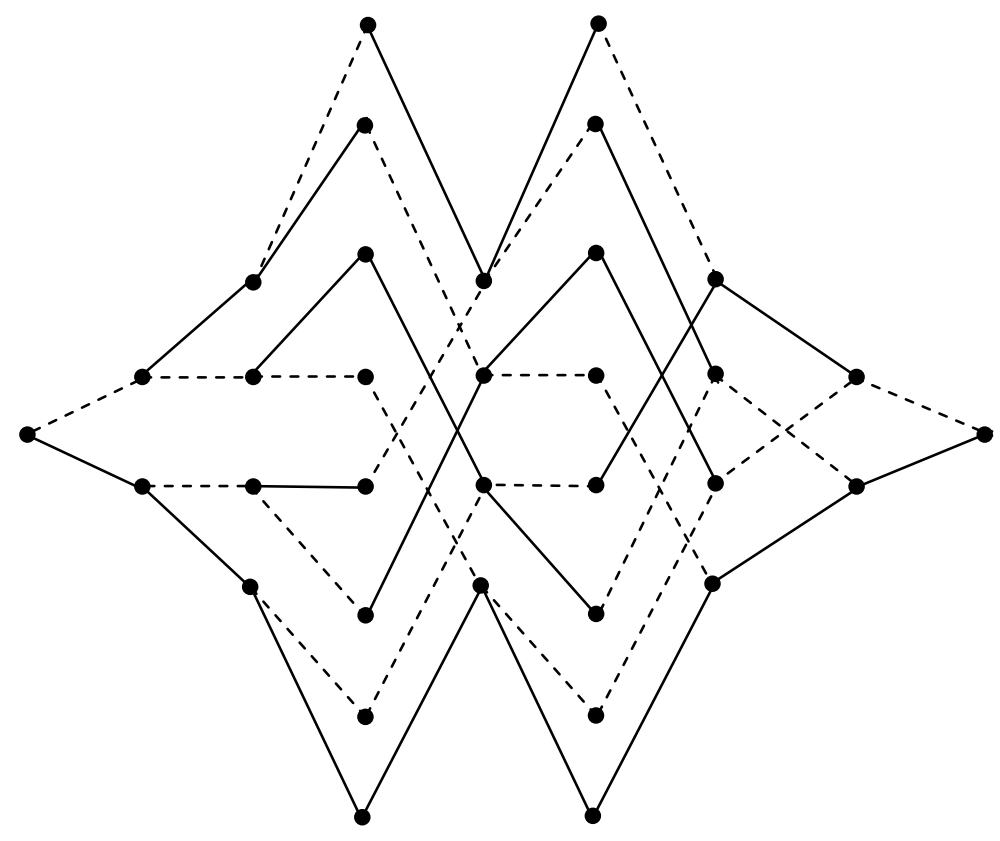

Figure 8.4: The minimal trellis of $\mathbf{G}_{4}$

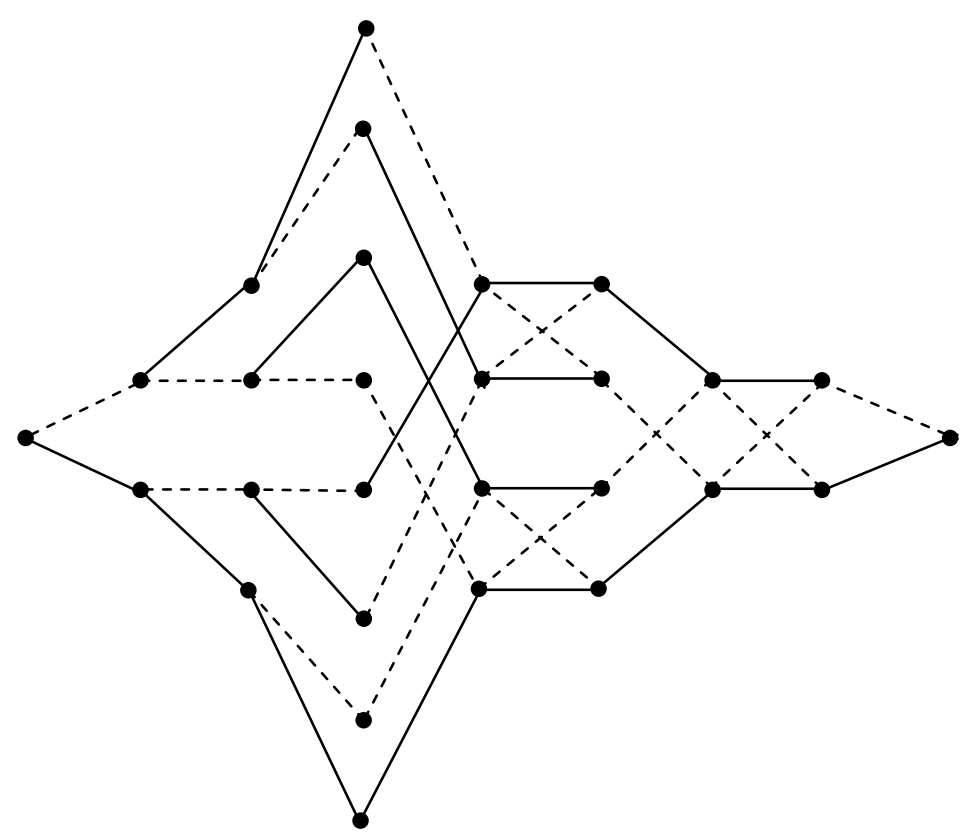

Figure 8.5: The minimal trellis of $\mathbf{G}_{5}$ 


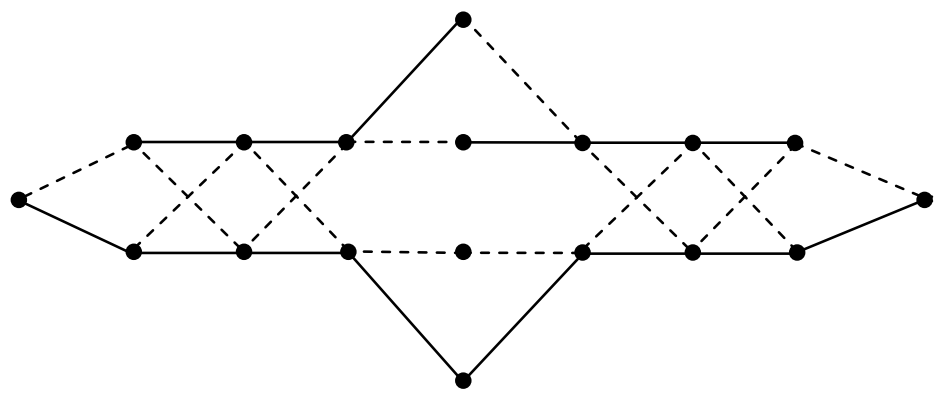

Figure 8.6: The minimal trellis of $\mathbf{G}_{6}$

simply finds the code bit with the minimum absolute value of LLR and flips its hard decision value if the parity check equation fails. For the rate $8 / 8$ the outer code is an identity matrix for which the received LLRs are used for making decisions. 


\section{List of References}

[1] S. Lin and P. S. Yu, "A hybrid ARQ scheme with parity retransmission for error control of satellite channels," IEEE Trans. Commun., vol. 30, no. 7, pp. 1701-1719, Jul. 1982.

[2] J. Hagenauer, "Rate-compatible punctured convolutional codes (RCPC codes) and their applications," IEEE Trans. Commun., vol. 36, no. 4, pp. 389-400, Apr. 1988.

[3] Z. Lin and A. Svensson, "New rate-compatible repetition convolutional codes," IEEE Trans. Inf. Theory, vol. 46, no. 7, pp. 2651-2659, Nov. 2000.

[4] S. Lin, D. J. Costello, and M. J. Miller, "Automatic repeat-request error control schemes," IEEE Commun. Mag., vol. 12, pp. 5-17, Dec. 1984.

[5] D. M. Mandelbaum, "An adaptive-feedback coding scheme using incremental redundancy," IEEE Trans. Inf. Theory, vol. 20, no. 3, pp. 388-389, May 1974.

[6] J. B. Cain, G. C. Clark, and J. M. Geist, "Punctured convolutional codes of rate (n$l) / n$ and simplified maximum likelihood decoding," IEEE Trans. Inf. Theory, vol. 25, no. 1, pp. 97-100, Jan. 1979.

[7] T. Rodrigues, R. D. Souza and M. E. Pellenz, "Hybrid ARQ scheme based on recursive convolutional codes and turbo decoding," in Proc. IEEE International Conference on Communications (ICC), Glasgow, Scotland, Jun. 2007.

[8] R. D. Souza, M. E. Pellenz and T. Rodrigues, "Hybrid ARQ scheme based on recursive convolutional codes and turbo decoding," IEEE Trans. Commun., vol. 57, no. 2, pp. 315-318, Feb. 2009.

[9] S. Kallel and D. Haccoun, "Generalized type II hybrid ARQ scheme using punctured convolutional coding," IEEE Trans. Commun., vol. 38, no. 11, pp. 1938-1946, Nov 1990.

[10] S. Falahati, T. Ottosson, A. Svensson and Lin Zihuai, "Convolutional coding and decoding in hybrid type-II ARQ schemes on wireless channels," in Proc. IEEE Vehicular Technology Conference(VTC), Houston, TX, May 1999.

[11] R. Mantha and F. R. Kschischang, "A capacity-approaching hybrid ARQ scheme using turbo codes," in Proc. IEEE Global Telecommunications Conf. (GLOBECOM), 1999. 
[12] D. N. Rowitch and L. B. Milstein, "On the performance of hybrid FEC/ARQ systems using rate compatible punctured turbo (RCPT) codes," IEEE Trans. Commun., vol. 48, pp. 94817959, Jun. 2000

[13] J. M. Shea, "Reliability-based hybrid ARQ," IEE Electronics Letters, vol. 38, no. 13, pp. 644-645, Jun. 2002.

[14] A. Roongta and J. M. Shea, "Reliability-based hybrid ARQ using convolutional codes," in Proc. IEEE Intl. Conf. on Commun. (ICC), Anchorage, Alaska, May 2003, pp. 2889-2893.

[15] A. Roongta, J. W. Moon and J. M. Shea, "Reliability-based hybrid ARQ as an adaptive response to jamming," IEEE Journal on Selected Areas in Commun., vol. 23, no. 5, pp. 1045-1055, May 2005.

[16] A. Roongta and J. M. Shea, "Reliability-based hybrid ARQ and rate-compatible punctured convolutional (RCPC) codes," in Proc. IEEE Wireless Commun. Net. Conf. (WCNC), Mar. 2004.

[17] R. G. Gallager, Low-density parity-check codes, Ph.D. dissertation, Dept. Elect. Eng., MIT., Cambridge, MA, 1963.

[18] F. Huang, X. Yi and T. Wang, "Reliability-based selective repeat hybrid ARQ protocol on low density parity check codes," Intl. Conf. on Artificial Reality and TelexistenceWorkshops (ICAT), Hangzhou, China, Dec. 2006.

[19] C. Xu, Y. Chang, Xin Zhang and D. Yang, "Novel reliability-based HARQ scheme for irregular LDPC codes," in Proc. IEEE Youth conf. on Inf. Comput. Telecommun.(YCICT), Beijing, China, Sept. 2009.

[20] D. G. Brennan, "Linear diversity combining techniques," in Proc. of the IEEE, vol. 91, no. 2, pp. 331-356, Feb 2003.

[21] T. J. Richardson, A. M. Shokrollahi and R. L. Urbanke, "Design of capacityapproaching irregular low-density parity-check codes," IEEE Trans. Inf. Theory, vol. 47, no. 2, pp. 619-637, Feb. 2001.

[22] S. Sesia, G. Caire and G. Vivier, "Incremental redundancy hybrid ARQ schemes based on low-density parity-check codes," IEEE Trans. Commun., vol. 52, pp. 1311-1321, Aug. 2004.

[23] R. Tanner, "A recursive approach to low complexity codes," IEEE Trans. Inf. Theory, vol. 27, no. 5, pp. 533-547, Sep. 1981.

[24] M. R. Yazdani and A.H. Banihashemi, "On construction of rate-compatible lowdensity parity-check codes," IEEE Commun. Lett., vol. 8, pp. 159-161, Mar. 2004.

[25] M. R. Yazdani and A.H. Banihashemi, "Irregular rate-compatible LDPC codes for capacity approaching hybrid ARQ schemes," in Proc. IEEE Canadian Conf. Elec. Comp. Engg., Niagara Falls, Canada, May 2004. 
[26] M. R. Yazdani and A.H. Banihashemi, "On construction of rate-compatible lowdensity parity-check codes," in Proc. IEEE International Conference on Communications (ICC), Paris, France, Jun. 2004.

[27] C. Di, D. Proietti, I. E. Telatar, T. J. Richardson, and R. L. Urbanke, "Finite-length analysis of low-density parity-check codes on the binary erasure channel," IEEE Trans. Inf. Theory, vol. 48, no. 6, pp. 1570171579, Jun. 2002

[28] T. Tian, C. Jones, and J. D. Villasenor, "Rate-compatible low-density paritycheckcodes," in Proc. Int. Symp. Information Theory, Chicago, IL, Jun./Jul. 2004.

[29] J. Ha and S. W. McLaughlin, "Optimal puncturing of irregular low-density paritycheck codes," in Proc. IEEE International Conference on Communications (ICC), 2003.

[30] J. Ha and S. W. McLaughlin, "Optimal puncturing distributions for rate-compatible low-density parity-check codes," in Proc. IEEE Intl. Symp. on Inf. Theory, Yokohama, Japan, Jun./Jul. 2003.

[31] J. Ha, J. Kim, and S. W. McLaughlin, "Rate-compatible puncturing of low-density parity-check codes," IEEE Trans. Inf. Theory, vol. 50, no. 11, pp. 2824-2836, Nov. 2004.

[32] J. Ha, J. Kim and S. W. McLaughlin, "Puncturing for finite length low-density paritycheck codes," Int. Symp. Information Theory, Chicago, IL, Jun./Jul. 2004.

[33] J. Ha, J. Kim, D. Klinc, S. W. McLaughlin, "Rate-compatible punctured low-density parity-check codes with short block lengths," IEEE Trans. Inf. Theory, vol. 52, no. 2, pp. 728-738, Feb. 2006

[34] B. N. Vellambi, F. Fekri, "Finite-length rate-compatible LDPC codes: A novel puncturing scheme," IEEE Trans. Commun., vol.57, no.2, pp.297-301, Feb. 2009.

[35] H. Pishro-Nik and F. Fekri, "Results on punctured low-density parity check codes and improved iterative decoding techniques," in Proc. Inf. Theory Workshop, San Antonio, Tx, USA, Oct. 2004.

[36] N. Varnica, E. Soljanin and P. Whiting, "LDPC code ensembles for incremental redundancy hybrid ARQ," in Proc. Int. Symp. on Inf. theory (ISIT), Adelaide, SA, Sept. 2005.

[37] J. Li and K. Narayanan, "Rate-compatible low density parity check codes for capacity approaching ARQ scheme in packet data communications," in Proc. Int. Conf. Comm., Internet, and Info. Tech. (CIIT), St. Thomas, US Virgin Islands, pp. 376-132, Nov., 2002.

[38] X.-Y. Hu, E. Eleftheriou and D. M. Arnold, "Regular and irregular progressive edgegrowth Tanner graphs," IEEE Trans. Inf. Theory, vol. 51, no. 1, pp. 386-398, Jan. 2005.

[39] M. Good and F. R. Kschischang, "Incremental redundancy via check splitting," in Proc. 23rd Biennial Symp. Commun., Kigston, ON, Jun. 2006 
[40] H. G. Joo, D. J. Shin and S. N. Hong, "New construction of rate-compatible blocktype low-density parity-check codes using splitting," in Proc. IEEE 17th Int. Symp. Personal, Indoor and Mobile Radio Commun., Helsinki, Finland, Sep. 2006.

[41] H. J. Joo, S. N. Hong and D. J. Shin, "Design of rate-compatible RA-type low-density parity-check codes using splitting," IEEE Trans. Commun., vol. 57, no. 12, pp. 35243528, Dec. 2009.

[42] S. X. Wu and W. H. Mow, "Constructing rate-compatible LDPC codes with a novel efficient ranking criterion," in Proc. IEEE Wireless Commun. Netw. Conf., Sydney, Australia, Apr. 2010.

[43] B. Young, S. Mhaske and P. Spasojevic, "Rate compatible IRA codes using row splitting for 5G wireless," in Proc. 49th Ann. Conf. Inf. Sci. Sys. (CISS), Baltimore, MD, Mar. 2015.

[44] H. Li and I. D. Marsland, "A comparison of rateless codes at short block lengths," in Proc. IEEE Int. Conf. on Commun., Beijing, China, May 2008.

[45] J. Garcia-Frias and Z. Wei, "Approaching Shannon performance by iterative decoding of linear codes with low-density generator matrix," IEEE Commun. Lett., vol. 7, pp. 26617268 , Jun. 2003.

[46] D. J. C. Mackay, "Good error-correcting codes based on very sparse matrices," IEEE Trans. Inf. Theory, vol. 45, no. 2, pp. 399-431, Mar. 1999.

[47] H. Lou and J. Garcia-Frias, "Rate-compatible low-density generator matrix codes," IEEE Trans. Commun., vol. 56, no. 3, pp. 321-324, Mar. 2008.

[48] M. Luby, "LT codes," in Proc. 43rd Annu. IEEE Symp. Foundations of Computer Science (FOCS), Vancouver, BC, Canada, Nov. 2002.

[49] A. Shokrollahi, "Raptor codes," IEEE Trans. Inf. Theory, vol. 52, no. 6, pp. 25512567, Jun. 2006.

[50] D. J. C. MacKay, "Fountain codes," IEE Proceedings Communications, vol. 152, no. 6, pp.1062-1068, Dec. 2005.

[51] C. Sae-Young, T. J. Richardson and R. L. Urbanke, "Analysis of sum-product decoding of low-density parity-check codes using a Gaussian approximation," IEEE Trans. Inf. Theory, vol. 47, no. 2, pp. 657-670, Feb. 2001.

[52] O. Etesami and A. Shokrollahi, "Raptor codes on binary memoryless symmetric channels,” IEEE Trans. Inf. Theory, vol.52, no. 5, pp. 2033-2051, May 2006.

[53] E. Soljanin , N. Varnica and P. Whiting, "Punctured vs rateless codes for hybrid ARQ," in Proc. Inf. theory Workshop(ITW), pp.155,159, 13-17 March 2006.

[54] E. Arikan, "Channel polarization: a method for constructing capacity-achieving codes for symmetric binary-input memoryless channels," IEEE Trans. Inf. Theory, vol. 55, pp. 3051-3073, Jul. 2009. 
[55] E. Arikan, "Channel polarization: A method for constructing capacity-achieving codes," in Proc. IEEE Int. Symp. Inf. Theory(ISIT), Toronto, ON, Jul. 2008.

[56] E. Sasoglu, E. Telatar and E. Arikan, "Polarization for arbitrary discrete memoryless channels," in Proc. IEEE Inf. Theory Workshop(ITW), Taormina, Italy, Oct. 2009.

[57] E. Arikan, "A performance comparison of polar codes and Reed-Muller codes," IEEE Commun. Lett., vol. 12, pp. 447-449, Jun. 2008.

[58] I. Tal and A. Vardy, "List decoding of polar codes," IEEE Trans. Inf. Theory, vol. 61, no. 5, pp. 2213-2226, May 2015.

[59] I. Tal and A. Vardy, "List decoding of polar codes," in Proc. Int. Symp. Inf. Theory (ISIT), St. Petersburg, Russia, July 2011.

[60] K. Chen, K. Niu and J. R. Lin, "List successive cancellation decoding of polar codes," Electronics Letters, vol. 48, pp. 500-501, Apr. 2012.

[61] K. Niu and K. Chen, "Stack decoding of polar codes," Electronics Letters, vol. 48, pp. 695-697, June 2012.

[62] K. Chen, K. Niu and J. Lin, "Improved successive cancellation decoding of polar codes," IEEE Trans. Commun., vol. 61, pp. 3100-3107, Aug. 2013.

[63] K. Niu and K. Chen, "CRC-aided decoding of polar codes," IEEE Commun. Lett., vol. 16, pp. 1668-1671, Oct. 2012.

[64] K. Chen, B. Li, H. Shen, J. Jin and D. Tse, "Reduce the complexity of list decoding of polar codes by tree-pruning," IEEE Commun. Letters, vol. 20, no. 2, pp. 204-207, Feb. 2016.

[65] K. Chen, K. Niu and J. Lin, "A Reduced-Complexity Successive Cancellation List Decoding of Polar Codes," in Proc. IEEE Vehicular Tech. Conf.(VTC Spring), Dresden, Jun. 2013.

[66] N. Hussami, S. B. Korada and R. Urbanke, "Performance of polar codes for channel and source coding," Int. Symp. Inf. Theory (ISIT), Seoul, Korea, June 2009.

[67] K. Niu, K. Chen, and J. Lin,"Beyond turbo codes: Rate-compatible punctured polar codes," in Proc. IEEE Int. Conf. on Commun. (ICC), Budapest, Jun. 2013, pp. 34233427.

[68] A. Eslami and H. Pishro-Nik, "A practical approach to polar codes," Online:arXiv:1107.5355v1, 26 Jul. 2011.

[69] L. Zhang, Z. Zhang, X. Wang, Q. Yu and Y. Chen, "On the puncturing patterns for punctured polar codes," in Proc. IEEE Intl. Symp. Inf. Theory (ISIT), Honolulu, HI, Jun./Jul. 2014.

[70] S. N. Hong, D. Hui and I. Maric, "Capacity-achieving rate-compatible polar codes," in Proc. IEEE Int. Symp. Inf. Theory (ISIT), Barcelona, Spain, July 2016. 
[71] K. Chen, K. Niu and J. Lin, "A hybrid ARQ scheme based on polar codes," IEEE Commun. Lett., vol. 17, no. 10, pp. 1996-1999, Oct. 2013.

[72] G. Yue, X. Wang, and M. Madihian, "Design of rate-compatible irregular repeat accumulate codes," IEEE Trans. Commun., vol. 55, no. 6, pp. 1153-1163, Jun. 2007.

[73] R. Mori and T. Tanaka, "Performance of polar codes with the construction using density evolution," IEEE Commun. Lett., vol. 13, no. 7, pp. 519-521, Jul. 2009.

[74] R. Mori and T. Tanaka, "Performance and construction of polar codes on symmetric binary-input memoryless channels," in Proc. IEEE Intl. Symp. Inf. Theory(ISIT), Seoul, South Korea, Jun./Jul. 2009.

[75] I. Tal and A. Vardy, "How to construct polar codes," IEEE Trans. Inf. Theory, vol. 59, no. 10 , pp. 6562-6582, Oct. 2013.

[76] H. Li and J. Yuan, "A practical construction method for polar codes in AWGN channels," in Proc. IEEE TENCON Spring Conf, Sydney, NSW, Apr. 2013

[77] D. Kern, S. Vorkoper and V. Kuhn, "A new code construction for polar codes using min-sum density," in Proc. IEEE Intl. Symp. Turbo Codes and Iterative Inf. Proc. (ISTC), Bremen, Germany, Aug. 2014.

[78] H. Vangala, E. Viterbo and Y. Hong, "A comparative study of polar code constructions for the AWGN channel", arXiv:1501.02473.

[79] J. Guo, M. Qin, A. G. Fabregas and P. H. Siegel, "Enhanced belief propagation decoding of polar codes through concatenation," in Proc. Int. Symp. Inf. Theory (ISIT), Honolulu, USA, Jun. 2014.

[80] S. B. Korada, E. Sasoglu and R. Urbanke, "Polar codes: Characterization of exponent, bounds, and constructions," IEEE Trans. Inf. Theory, vol. 56, pp. 6253-6264, Dec. 2010.

[81] V. Miloslavskaya and P. Trifonov, "Design of binary polar codes with arbitrary kernel," in Proc. Inf. Theory Workshop (ITW), Lausanne, Switzerland, Sept. 2012.

[82] H. Mahdavifar, M. El-Khamy, J. Lee and I. Kang, "On the construction and decoding of concatenated polar codes," Proc. Int. Symp. Inf. Theory (ISIT), Istanbul, Turkey, Jul. 2013.

[83] M. Seidl and J. B. Huber, "Improving successive cancellation decoding of polar codes by usage of inner block codes," Proc. Int. Symp. Turbo Codes Iterative Inf. Process., Brest, France, Sep. 2010.

[84] P. Trifonov and P. Semenov, "Generalized concatenated codes based on polar codes," Proc. Int. Symp. Wireless Commun. Systems, Aachen, Germany, Nov. 2011.

[85] I. Dumer, "Nested polarized codes," in Proc. Int. workshop. Algebraic Combinatorial Coding Theory, Svetlogorsk, Russia, Sep. 2014.

[86] P. Trifonov, "Efficient design and decoding of polar codes," IEEE Trans. Inf. Theory, vol. 60, pp. 3221-3227, Nov. 2012. 
[87] M. Grassl, Bounds on the minimum distance of linear codes and quantum codes." Available: http://www.codetables.de, 2007.

[88] M. Grassl, "Searching for linear codes with large minimum distance," Discovering Mathematics with Magma Reducing the Abstract to the Concrete, Algorithms and Computation in Mathematics, Springer, vol. 19, pp. 287-313, 2006

[89] A. B. Kiely, S. J. Dolinar, R. J. McEliece, L. Ekroot and W. Lin, “Trellis decoding complexity of linear block codes," IEEE Trans. Inf. Theory, vol. 42, pp. 1687-1697, Nov. 1996. 